EWERTON RODRIGUES ANDRADE

\title{
LYRA2: PASSWORD HASHING SCHEME WITH IMPROVED SECURITY AGAINST TIME-MEMORY TRADE-OFFS
}

\section{LYRA2: UM ESQUEMA DE HASH DE SENHAS COM MAIOR SEGURANÇA CONTRA TRADE-OFFS ENTRE PROCESSAMENTO E MEMÓRIA}

\footnotetext{
Tese apresentada à Escola Politécnica da Universidade de São Paulo para obtenção do Título de Doutor em Ciências.
} 
EWERTON RODRIGUES ANDRADE

\section{LYRA2: PASSWORD HASHING SCHEME WITH IMPROVED SECURITY AGAINST TIME-MEMORY TRADE-OFFS}

\section{LYRA2: UM ESQUEMA DE HASH DE SENHAS COM MAIOR SEGURANÇA CONTRA TRADE-OFFS ENTRE PROCESSAMENTO E MEMÓRIA}

Tese apresentada à Escola Politécnica da Universidade de São Paulo para obtenção do Título de Doutor em Ciências.

Área de Concentração:

Engenharia de Computação

Orientador:

Prof. Dr. Marcos A. Simplicio Junior 
Este exemplar foi revisado e corrigido em relação à versão original, sob responsabilidade única do autor e com a anuência de seu orientador.

São Paulo, de de

Assinatura do autor:

Assinatura do orientador:

Andrade, Ewerton Rodrigues

Lyra2: Um Esquema de Hash de Senhas com maior segurança contra trade-offs entre processamento e memória (Lyra2: Password Hashing Scheme with improved security against time-memory trade-offs) / E. R. Andrade -versão corr. -- São Paulo, 2016.

$139 \mathrm{p}$.

Tese (Doutorado) - Escola Politécnica da Universidade de São Paulo. Departamento de Engenharia de Computação e Sistemas Digitais.

1.Metodologia e técnicas de computação 2.Segurança de computadores 3.Criptologia 4.Algoritmos 5.Esquemas de Hash de Senhas I.Universidade de São Paulo. Escola Politécnica. Departamento de Engenharia de Computação e Sistemas Digitais II.t. 
"In practice, the effectiveness of a countermeasure often depends on how it is used; the best safe in the world is worthless if no one remembers to close the door."

Computer at Risk, 1991. 


\section{AGRADECIMENTOS}

Primeiramente, agradeço a duas pessoas extremamente importantes na minha vida: meu pai, Carlos Lopes Andrade, e minha mãe, Terezinha Aparecida Rodrigues Andrade (mãe Nega). A vocês o meu muito obrigado por me ajudarem a enfrentar todos os desafios e transpor meus limites, sempre me apoiando, mesmo que muitas vezes tenham se (e me) perguntado quando é que eu iria "começar a trabalhar".

A minha irmã, Karla Rodrigues Andrade, pelo sempre presente apoio, compreensão e demonstração de carinho.

Também agradeço imensamente ao meu orientador, professor e amigo, Marcos Antonio Simplicio Junior, sempre disponível para longas conversas sobre qualquer que fosse o assunto. Obrigado pela amizade, pelo exemplo, e sobretudo por confiar em mim e me auxiliar durante o desenvolvimento desta tese com seu conhecimento e experiência.

Aos membros da banca de qualificação e da comissão julgadora, pelos questionamentos, correções, sugestões e comentários.

Aos amigos e membros do Laboratório de Arquitetura de Redes de Computadores (LARC), e também à Prof. ${ }^{a}$ Tereza Carvalho e ao Prof. Wilson Ruggiero, amigos e coordenadores de diversos projetos dos quais participei. Muito obrigado pelo companheirismo, conhecimento compartilhado, e pelas inspiradas e bem humoradas discussões.

A todos demais professores, alunos e funcionários da Escola Politécnica da Universidade de São Paulo que colaboraram com minha formação, viabilizando a produção desta obra.

À FDTE (Fundação Para o Desenvolvimento Tecnológico da Engenharia) e à CAPES (Coordenação de Aperfeiçoamento de Pessoal de Nível Superior) pelo auxílio financeiro.

A todos os amigos fiéis que, apesar de muitas vezes separados por quilômetros de distância, continuam sempre disponíveis para uma conversa pelo simples prazer da companhia uns dos outros. E a todos os demais que conviveram comigo durante o desenvolvimento deste trabalho.

Meus sinceros agradecimentos a todos vocês. 


\section{RESUMO}

Para proteger-se de ataques de força bruta, sistemas modernos de autenticação baseados em senhas geralmente empregam algum Esquema de Hash de Senhas (Password Hashing Scheme - PHS). Basicamente, um PHS é um algoritmo criptográfico que gera uma sequência de bits pseudo-aleatórios a partir de uma senha provida pelo usuário, permitindo a este último configurar o custo computacional envolvido no processo e, assim, potencialmente elevar os custos de atacantes testando múltiplas senhas em paralelo. Esquemas tradicionais utilizados para esse propósito são o PBKDF2 e bcrypt, por exemplo, que incluem um parâmetro configurável que controla o número de iterações realizadas pelo algoritmo, permitindo ajustar-se o seu tempo total de processamento. Já os algoritmos scrypt e Lyra, mais recentes, permitem que usuários não apenas controlem o tempo de processamento, mas também a quantidade de memória necessária para testar uma senha. Apesar desses avanços, ainda há um interesse considerável da comunidade de pesquisa no desenvolvimento e avaliação de novas (e melhores) alternativas. De fato, tal interesse levou recentemente à criação de uma competição com esta finalidade específica, a Password Hashing Competition (PHC). Neste contexto, o objetivo do presente trabalho é propor uma alternativa superior aos PHS existentes. Especificamente, tem-se como alvo melhorar o algoritmo Lyra, um PHS baseado em esponjas criptográficas cujo projeto contou com a participação dos autores do presente trabalho. O algoritmo resultante, denominado Lyra2, preserva a segurança, eficiência e flexibilidade do Lyra, incluindo a habilidade de configurar do uso de memória e tempo de processamento do algoritmo, e também a capacidade de prover um uso de memória superior ao do scrypt com um tempo de processamento similar. Entretanto, ele traz importantes melhorias quando comparado ao seu predecessor: (1) permite um maior nível de segurança contra estratégias de ataque envolvendo trade-offs entre tempo de processamento e memória; (2) inclui a possibilidade de elevar os custos envolvidos na construção de plataformas de hardware dedicado para ataques contra o algoritmo; (3) e provê um equilíbrio entre resistância contra ataques de canal colateral ("side-channel") e ataques que se baseiam no uso de dispositivos de memória mais baratos (e, portanto, mais lentos) do que os utilizados em computadores controlados por usuários legítimos. Além da descrição detalhada do projeto do algoritmo, o presente trabalho inclui também uma análise detalhada de sua segurança e de seu desempenho em diferentes plataformas. Cabe notar que o Lyra2, conforme aqui descrito, recebeu uma menção de reconhecimento especial ao final da competição PHC previamente mencionada.

Palavras-chave: derivação de chaves, senhas, autenticação de usuários, segurança, esponjas criptográficas. 


\section{ABSTRACT}

To protect against brute force attacks, modern password-based authentication systems usually employ mechanisms known as Password Hashing Schemes (PHS). Basically, a PHS is a cryptographic algorithm that generates a sequence of pseudorandom bits from a user-defined password, allowing the user to configure the computational costs involved in the process aiming to raise the costs of attackers testing multiple passwords trying to guess the correct one. Traditional schemes such as PBKDF2 and bcrypt, for example, include a configurable parameter that controls the number of iterations performed, allowing the user to adjust the time required by the password hashing process. The more recent scrypt and Lyra algorithms, on the other hand, allow users to control both processing time and memory usage. Despite these advances, there is still considerable interest by the research community in the development of new (and better) alternatives. Indeed, this led to the creation of a competition with this specific purpose, the Password Hashing Competition (PHC). In this context, the goal of this research effort is to propose a superior PHS alternative. Specifically, the objective is to improve the Lyra algorithm, a PHS built upon cryptographic sponges whose project counted with the authors' participation. The resulting solution, called Lyra2, preserves the security, efficiency and flexibility of Lyra, including: the ability to configure the desired amount of memory and processing time to be used by the algorithm; and (2) the capacity of providing a high memory usage with a processing time similar to that obtained with scrypt. In addition, it brings important improvements when compared to its predecessor: (1) it allows a higher security level against attack venues involving time-memory trade-offs; (2) it includes tweaks for increasing the costs involved in the construction of dedicated hardware to attack the algorithm; (3) it balances resistance against side-channel threats and attacks relying on cheaper (and, hence, slower) storage devices. Besides describing the algorithm's design rationale in detail, this work also includes a detailed analysis of its security and performance in different platforms. It is worth mentioning that Lyra2, as hereby described, received a special recognition in the aforementioned PHC competition.

Keywords: Password-based key derivation, passwords, authentication, security, cryptographic sponges. 


\section{RIASSUNTO}

Per proteggersi da attacchi di forza bruta, moderni sistemi di autenticazione basati su password in generale impiegano qualche schema di password hash (Password Hashing Scheme - PHS). Fondamentalmente un PHS è un algoritmo crittografico che genera una sequenza di bit pseudocasuali da una password impostata dall'utente, permettendo a quest'ultimo di impostare il costo computazionale coinvolti nel processo e quindi potenzialmente elevando i costi per attaccare utilizzando un set di password in parallelo. Schemi tradizionali usate per questo scopo sono PBKDF2 e bcrypt, per esempio. Questi schemi includdono un parametro configurabile che controlla il numero di iterazioni eseguite dall'algoritmo, permettendo di regolare il loro tempo di lavorazione totale. Invece, gli algoritmi scrypt e Lyra, più recente, consente agli utenti di controllare non solo il tempo di lavorazione, ma anche la quantità di memoria necessaria per la prova di una password. Nonostante questi progressi, c'è ancora un notevole interesse della comunità di ricerca per lo sviluppo e la valutazione di nuovi (e migliori) alternative. Infatti, questo interesse ha recentemente portato alla creazione di una competizione con questo specifico scopo, Password Hashing Competition (PHC). In questo contesto, l'obiettivo di questo lavoro è quello di proporre un'alternativa superiore ai PHS esistenti. In particolare, l?obiettivo è migliorare l'algoritmo Lyra, un PHS basato in spugne crittografici, il cui progetto ha avuto la partecipazione dagli autori di questa tesi. L'algoritmo risultante, chiamato Lyra2, conserva la sicurezza, l'efficienza e la flessibilità della Lyra, tra cui la possibilità di configurare l'utilizzo della memoria e tempo di lavorazione del algoritmo, e anche la capacità di fornire un utilizzo di memoria superiore al scrypt con un tempo di lavorazione simile. Tuttavia, porta miglioramenti significativi rispetto al suo predecessore: (1) permette un più elevato livello di sicurezza contro le strategie di attacco che coinvolgono trade-off tra tempo di lavorazione e di memoria; (2) prevede la possibilità di aumentare i costi di costruzione di piattaforme hardware dedicate al attacchi contro l'algoritmo; (3) e fornisce un equilibrio tra resistenza contro canale laterale ("side-channel") e attacchi sono basati sull'uso di dispositivi più economici di memoria (e quindi più lento) di quelli utilizzati sui computer controllati da utenti legittimi. Oltre alla descrizione dettagliata della progettazione dell'algoritmo, questo studio comprende anche un'analisi dettagliata della sua sicurezza e le prestazioni su piattaforme diverse. E inportante notare che Lyra2 come qui descritto, ha ricevuto particolare riconoscimento nel concorso $\mathrm{PHC}$, precedentemente menzionato.

Parole chiave: derivazione di chiave, passwords, autenticazione degli utenti, sicurezza, spugne crittografici. 


\section{CONTENTS}

List of Figures $\quad x$

List of Tables $\quad$ xii

List of Acronyms xiii

List of Symbols $\quad$ XV

1 Introduction $\quad 16$

1.1 Motivation ......................... 17

1.2 Goals and Original Contributions . . . . . . . . . . . . 19

1.3 Methods . . . . . . . . . . . . . . . . . . . 20

1.4 Document Organization . . . . . . . . . . . . . . . . . . . . 21

2 Background $\quad 22$

2.1 Hash-Functions . . . . . . . . . . . . . . . . . . . . 22

2.1 .1 Security ........................ 23

2.2 Cryptographic Sponges . . . . . . . . . . . . . . . . . . . . . . . 24

2.2.1 Basic Structure . . . . . . . . . . . . . . . . . . . . 24

2.2.2 The duplex construction . . . . . . . . . . . . . 25

2.2 .3 Security . . . . . . . . . . . . . . . 26

2.3 Password Hashing Schemes (PHS) . . . . . . . . . . . . . . . 26 
2.3.1 Attack platforms . . . . . . . . . . . . . . . 28

2.3.1.1 Graphics Processing Units (GPUs) . . . . . . 28

2.3.1.2 Field Programmable Gate Arrays (FPGAs) . . . 29

3 Related Works $\quad 31$

3.1 Pre-PHC Schemes . . . . . . . . . . . . . . . . . . . 31

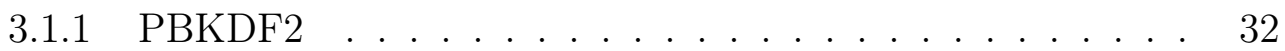

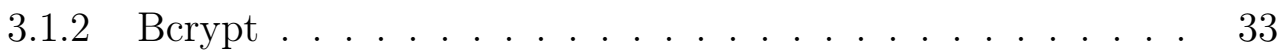

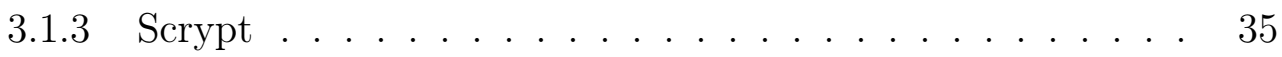

$3.1 .4 \quad$ Lyra . . . . . . . . . . . . . . . . . . . . 37

3.2 Schemes from $\mathrm{PHC} \ldots \ldots . \ldots . . \ldots . . \ldots 40$

$3.2 .1 \quad \operatorname{Argon} 2 \ldots \ldots \ldots . \ldots . \ldots . \ldots . \ldots 4$

3.2 .2 battcrypt .......................... 43

3.2.3 Catena ........................... 43

3.2 .4 POMELO . . . . . . . . . . . . . . . . . . . 45

3.2 .5 yescrypt ........................... 46

$\begin{array}{llr}4 & \text { Lyra2 } & 48\end{array}$

4.1 Structure and rationale . . . . . . . . . . . . . . 50

4.1.1 Bootstrapping . . . . . . . . . . . . . 50

4.1 .2 The Setup phase . . . . . . . . . . . . . 51

4.1.3 The Wandering phase . . . . . . . . . . . 55

4.1.4 The Wrap-up phase . . . . . . . . . . . . . . . 57 
4.2 Strictly sequential design . . . . . . . . . . . . . . . 57

4.3 Configuring memory usage and processing time . . . . . . . . 60

4.4 On the underlying sponge . . . . . . . . . . . . . . . . 60

4.4.1 A dedicated, multiplication-hardened sponge: BlaMka. . . 62

4.5 Practical considerations . . . . . . . . . . . . . . 63

$\begin{array}{lll}5 & \text { Security analysis } & 66\end{array}$

5.1 Low-Memory attacks . . . . . . . . . . . . . . . . 67

5.1 .1 Preliminaries . . . . . . . . . . . . . . . . . 69

5.1 .2 The Setup phase . . . . . . . . . . . . . 70

5.1.2.1 Storing only what is needed: $1 / 2$ memory usage . 71

5.1.2.2 Storing less than what is needed: $1 / 4$ memory usage 72

5.1.2.3 Storing less than what is needed: $1 / 8$ memory usage 74

5.1.2.4 Storing less than what is needed: generalization . 78

5.1.2.5 Storing only intermediate sponge states _. . . . 81

5.1.3 Adding the Wandering phase: consumer-producer strategy 84

5.1.3.1 The first $R / 2$ iterations of the Wandering phase with $1 / 2$ memory usage. . . . . . . . . . . 85

5.1.3.2 The whole Wandering phase with $1 / 2$ memory usage 88

5.1.3.3 The whole Wandering phase with less than $1 / 2$ memory usage . . . . . . . . . . . . . . 990

5.1.4 Adding the Wandering phase: sentinel-based strategy . . . 91 
5.1.4.1 On the (low) scalability of the sentinel-based strategy ........................ 94

5.2 Slow-Memory attacks . . . . . . . . . . . . . . . . . 97

5.3 Cache-timing attacks . . . . . . . . . . . . . . . . 99

5.4 Garbage-Collector attacks . . . . . . . . . . . . . 102

5.5 Security analysis of BlaMka. . . . . . . . . . . . . 103

5.6 Summary . . . . . . . . . . . . . . . . . . . . . 104

6 Performance for different settings 106

6.1 Benchmarks for Lyra2 . . . . . . . . . . . . . . 106

6.2 Benchmarks for BlaMka. . . . . . . . . . . . . . . . 111

6.3 Benchmarks for Lyra2 with BlaMka . . . . . . . . . . . . . . . 115

6.4 Expected attack costs . . . . . . . . . . . . 116

6.5 Summary . . . . . . . . . . . . . . . . . 117

$\begin{array}{llr}7 & \text { Final Considerations } & 119\end{array}$

7.1 Publications and other results . . . . . . . . . . . . . 119

7.2 Future works . . . . . . . . . . . . . . . . . . . 121

$\begin{array}{lr}\text { References } & 122\end{array}$

$\begin{array}{lr}\text { Appendix A. Naming conventions } & 132\end{array}$

Appendix B. Further controlling Lyra2's bandwidth usage 133

Appendix C. An alternative design for BlaMka: avoiding latency 135 


\section{LIST OF FIGURES}

1 Overview of the sponge construction $Z=[f$, pad, $b](M, \ell)$. Adapted from (BERTONI et al., 2011a). . . . . . . . . . . 24

2 Overview of the duplex construction. Adapted from (BERTONI et al., 2011a). . . . . . . . . . . . . .

$3 \quad$ Handling the sponge's inputs and outputs during the Setup (left) and Wandering (right) phases in Lyra2. . . . . . . . . . . . 53

4 BlaMka's multiplication-hardened (right) and Blake2b's original (left) permutations. . . . . . . . . . . . . . . . 62

$5 \quad$ The Setup phase. . . . . . . . . . . . . . . 71

$6 \quad$ Attacking the Setup phase: storing $1 / 2$ of all rows. . . . . . . . 71

$7 \quad$ Attacking the Setup phase: storing $1 / 4$ of all rows. . . . . . . . 73

8 Attacking the Setup phase: recomputing $M\left[6_{A}\right]$ while storing $1 / 8$ of all rows and keeping $M[F]$ in memory. . . . . . . . . . . . . 77

$9 \quad$ Attacking the Setup phase: storing only sponge states. . . . . . . 81

10 Reading and writing cells in the Setup phase. . . . . . . . . . . . 83

11 An example of the Wandering phase's execution. . . . . . . . . . . 84

12 Tree representing the dependence among rows in Lyra2. . . . . . . 89

13 Tree representing the dependence among rows in Lyra2 with $T=2$ : using $\epsilon^{\prime}$ sentinels per level. . . . . . . . . . . . . . . . . . . 95 
14 Performance of SSE-enabled Lyra2, for $C=256, \rho=1, p=1$, and different $T$ and $R$ settings, compared with SSE-enabled scrypt. 107

15 Performance of SSE-enabled Lyra2, for $C=256, \rho=1, p=1$, and different $T$ and $R$ settings, compared with SSE-enabled scrypt and memory-hard PHC finalists with minimum parameters. . . . . . . 108

16 Performance of SSE-enabled Lyra2, for $C=256, \rho=1, p=1$ and different $T$ and $R$ settings, compared with SSE-enabled scrypt and memory-hard PHC finalists with a similar number of calls to the underlying function. . . . . . . . . . . . . . . . . 109

17 Performance of SSE-enabled Lyra2 with BlaMka, for $C=256$, $\rho=1, p=1$, and different $T$ and $R$ settings, compared with SSE-enabled scrypt and memory-hard PHC finalists . . . . . . . . 115

18 Different permutations: Blake2b's original permutation (left), BlaMka's $G_{\mathrm{tls}}$ multiplication-hardened permutation (middle) and BlaMka's latency-oriented multiplication-hardened permutation $G_{\mathrm{tls} \oplus}$ (right). . . . . . . . . . . . . . . 135

19 Improving the latency of $G \ldots \ldots \ldots$. . . . . . . . . . 137 


\section{LIST OF TABLES}

1 Indices of the rows that feed the sponge when computing $M[$ row] during Setup (hexadecimal notation). . . . . . . . . . . 55

2 Security overview of the PHSs considered the state of the art. . . 105

3 PHC finalists: calls to underlying primitive in terms of their time and memory parameters, $T$ and $M$, and their implementations. . 110

4 Data related of the tests performed in CPU, executing just one round of $G$ function (i.e., 256 bits of output). . . . . . . . . . . 112

5 Data related of the initial tests performed in FPGA, executing just one round of $G$ function (i.e., 256 bits of output). . . . . . . . . . 114

6 Data related of the initial tests performed in dedicated hardware (that present advantage against $\mathrm{CPU}$ ), executing just one round of $G$ function (i.e., 256 bits of output). . . . . . . . . . . . 114

$7 \quad$ Memory-related cost (in U\$) added by the SSE-enable version of Lyra2 with $T=1$ and $T=5$, for attackers trying to break passwords in a 1-year period using an Intel Xeon E5-2430 or equivalent processor. ........................... 117 


\title{
LIST OF ACRONYMS
}

\author{
AES Advanced Encryption Standard \\ API Application Programming Interface \\ ASIC Application Specific Integrated Circuit \\ AVX Advanced Vector Extensions \\ bcrypt Blowfish crypt \\ CBC Cipher Block Chaining \\ CPU Central Processing Unit \\ CUDA Compute Unified Device Architecture \\ DDR3 Double Data Rate type 3 Synchronous DRAM \\ DIMM Dual Inline Memory Module \\ DRAM Dynamic Random Access Memory \\ FPGA Field-Programmable Gate Array \\ GPU Graphics Processing Unit \\ HMAC Hash-based Message Authentication Code \\ KDF Key Derivation Function \\ MMX Multiple Math eXtension or Matrix Math eXtension \\ NIST National Institute of Standards and Technology \\ OpenCL Open Computing Language \\ PBKDF2 Password-Based Key Derivation Function 2
}




$\begin{array}{ll}\text { PHC } & \text { Password Hashing Competition } \\ \text { PHP } & \text { Personal Home Page } \\ \text { PHS } & \text { Password Hashing Scheme } \\ \text { PKCS } & \text { Public-Key Cryptography Standards } \\ \text { RAM } & \text { Random Access Memory } \\ \text { SHA } & \text { Secure Hash Algorithm } \\ \text { SIMD } & \text { Single Instruction, Multiple Data } \\ \text { SSE } & \text { Streaming SIMD Extensions } \\ \text { SRAM } & \text { Static Random Access Memory } \\ \text { TMTO } & \text { Time-Memory trade-offs } \\ \text { XOR } & \text { Exclusive-OR operation }\end{array}$




\section{LIST OF SYMBOLS}

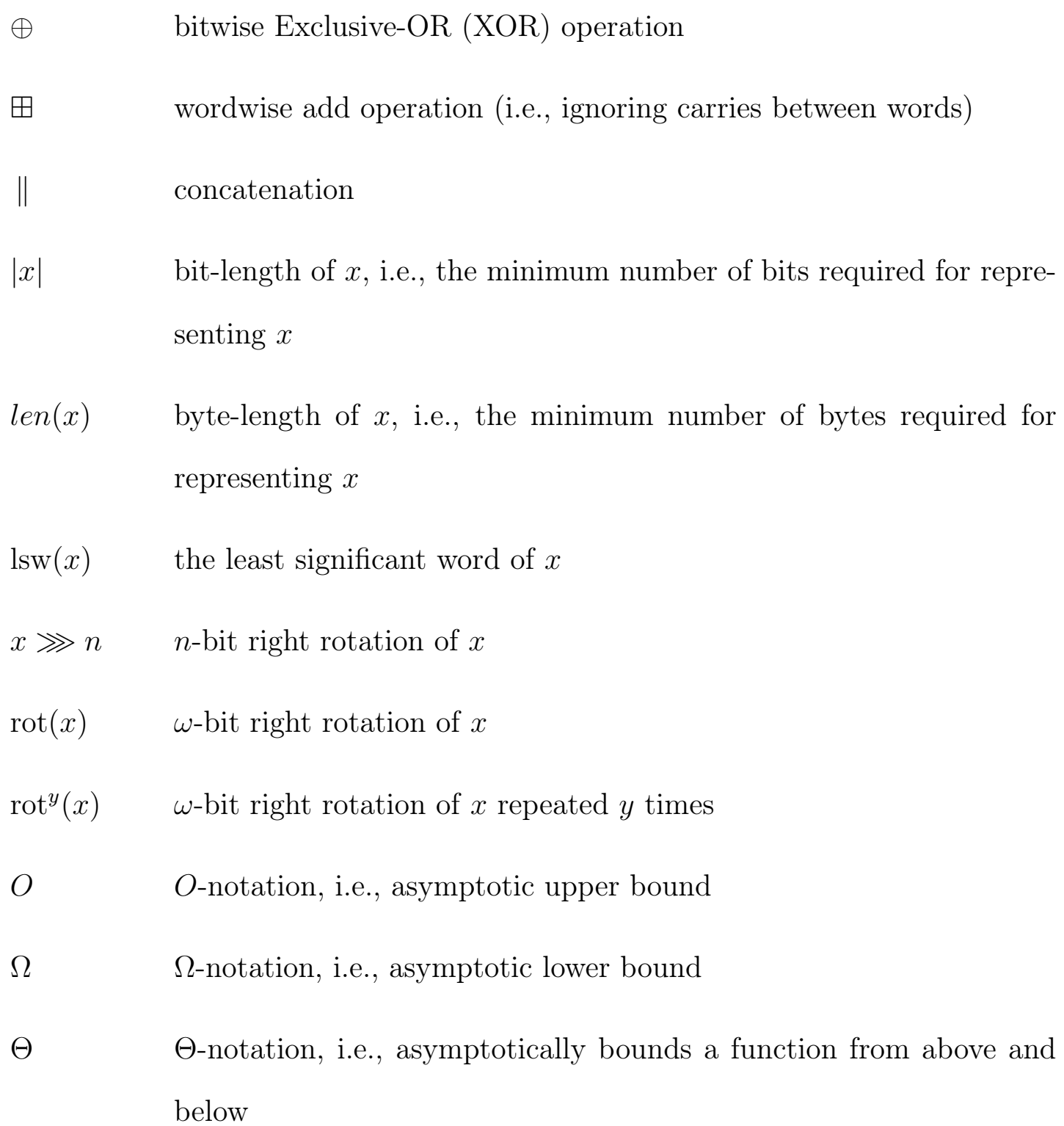




\section{INTRODUCTION}

User authentication is one of the most vital elements in modern computer security. Even though there are authentication mechanisms based on biometric devices ("what the user is") or physical devices such as smart cards ("what the user has"), the most widespread strategy still is to rely on secret passwords ("what the user knows"). This happens because password-based authentication remains as the most cost effective and efficient method of maintaining a shared secret between a user and a computer system (CHAKRABARTI; SINGBAL, 2007; CONKLIN; DIETRICH; WALZ, 2004). For better or for worse, and despite the existence of many proposals for their replacement (BONNEAU et al., 2012), this prevalence of passwords as one and commonly only factor for user authentication is unlikely to change in the near future.

Password-based systems usually employ some cryptographic algorithm that allows the generation of a pseudorandom string of bits from the password itself, known as a Password Hashing Scheme (PHS), or Key Derivation Function (KDF) (NIST, 2009). Typically, the output of the PHS is employed in one of two manners (PERCIVAL, 2009): it can be locally stored in the form of a "token" for future verifications of the password or used as the secret key for encrypting and/or authenticating data. Whichever the case, such solutions internally employ a oneway (e.g., hash) function, so that recovering the password from the PHS's output is computationally infeasible (PERCIVAL, 2009; KALISKI, 2000). 
Despite the popularity of password-based authentication, the fact that most users choose quite short and simple strings as passwords leads to a serious issue: they commonly have much less entropy than typically required by cryptographic keys (NIST, 2011). Indeed, a study from 2007 with 544,960 passwords from real users has shown an average entropy of approximately 40.5 bits (FLORENCIO; HERLEY, 2007), against the 128 bits usually required by modern systems. Such weak passwords greatly facilitate many kinds of "brute-force" attacks, such as dictionary attacks and exhaustive search (CHAKRABARTI; SINGBAL, 2007; HERLEY; OORSCHOT; PATRICK, 2009), allowing attackers to completely bypass the non-invertibility property of the password hashing process.

For example, an attacker could apply the PHS over a list of common passwords until the result matches the locally stored token or the valid encryption/authentication key. The feasibility of such attacks depends basically on the amount of resources available to the attacker, who can speed up the process by performing many tests in parallel. Such attacks commonly benefit from platforms equipped with many processing cores, such as modern GPUs (DÜRMUTH; GÜNEYSU; KASPER, 2012; SPRENGERS, 2011) or custom hardware (DÜRMUTH; GÜNEYSU; KASPER, 2012; MARECHAL, 2008).

\subsection{Motivation}

A straightforward approach for addressing this problem is to force users to choose complex passwords. This is unadvised, however, because such passwords would be harder to memorize and, thus, more easily forgotten or stolen due to the users' need of writing them down, defeating the whole purpose of authentication (CHAKRABARTI; SINGBAL, 2007). For this reason, modern password hashing solutions usually employ mechanisms for increasing the cost of brute force attacks. Schemes such as PBKDF2 (KALISKI, 2000) and bcrypt (PROVOS; MAZIÈRES, 
1999), for example, include a configurable parameter that controls the number of iterations performed, allowing the user to adjust the time required by the password hashing process.

A more recent proposal, scrypt (PERCIVAL, 2009), allows users to control both processing time and memory usage, raising the cost of password recovery by increasing the silicon space required for running the PHS in custom hardware, or the amount of RAM required in a GPU. Since this may raise the RAM costs of password cracking to unbearable levels, attackers may try to trade memory for processing time, discarding (parts of) the memory used and recomputing the discarded information when (and only when) it becomes necessary (PERCIVAL, 2009). The exploitation of such time-memory trade-offs (TMTO) leads to the hereby-called low-memory attacks. Another approach that might be used by attackers trying to reduce the costs of password cracking is to use low-cost (and, thus, slower) storage devices for keeping all memory used in the legitimate process, using the spare budget to run more tests in parallel and, thus, compensating the lower speed of each test; we call this approach a slow-memory attack.

Besides the need for protection against low- and slow-memory attacks, there is also interest in the development of solutions that are safe against side-channel attacks, especially the so-called cache-timing attacks. Basically, a cache-timing attack is possible if the attacker can observe a machine's timing behavior by monitoring its access to cache memory (e.g., the occurrence of cache-misses), building a profile of such occurrences for a legitimate password hashing process (FORLER; LUCKS; WENZEL, 2013; BERNSTEIN, 2005). Then, at least in theory, if the password being tested does not match the observed cache-timing behavior, the test could be aborted earlier, saving resources. Although this class of attack has not been effectively implemented in the context of PHSs, it has been shown to be effective, for example, against certain implementations of the Advanced Encryp- 
tion Standard (AES) (NIST, 2001a) and RSA (RIVEST; SHAMIR; ADLEMAN, 1978).

The considerable interest by the research community in developing new (and better) password hashing alternatives has recently even led to the creation of a cryptographic competition with this specific purpose, the Password Hashing Competition (PHC) (PHC, 2013).

\subsection{Goals and Original Contributions}

Aiming to address this need for stronger alternatives, our early studies led us to propose Lyra (ALMEIDA et al., 2014), a mode of operation of cryptographic sponges (BERTONI et al., 2007; BERTONI et al., 2011a) for password hashing. In this research, we propose an improved version of Lyra, simply called Lyra2.

Basically, Lyra2 preserves the flexibility and efficiency of Lyra, including:

1. The ability to configure the desired amount of memory and processing time to be used by the algorithm;

2. The capacity of providing a higher memory usage than that obtained with scrypt for a similar processing time.

In addition, it brings important security improvements when compared to its predecessor:

1. It allows a higher security level against attack venues involving timememory trade-offs (TMTO);

2. It includes tweaks to increase the costs involved in the construction of dedicated hardware for attacking the algorithm (e.g., FPGAs or ASICs); 
3. It balances resistance against side-channel threats and attacks relying on cheaper (and, hence, slower) storage devices.

For example, the processing cost of memory-free attacks against the algorithm grows exponentially with its time-controlling parameter, surpassing scrypt's quadratic growth in the same conditions. Hence, with a suitable choice of parameters, the attack approach of using extra processing for circumventing (part of) the algorithm's memory needs quickly becomes impractical. In addition, for an identical processing time, Lyra2 allows for a higher memory usage than its counterparts, further raising the costs of any possible attack venue.

\subsection{Methods}

The method adopted herein is the applied research based on the hypothesisdeduction approach, i.e., by using scientific references to define the problem, specify the solution hypotheses and, finally, evaluate them (WAZLAWICK, 2008).

For accomplishing this, the work was separated according to the following steps:

- Literature research: survey of existing PHS, based on the analysis of academic articles and technical manuals. This included a comparison between existing solutions and the evaluation of their internal structures, security and performance, making it possible to determine attractive approaches to create a novel algorithm;

- Design of algorithm: using the literature research as basis, this step consists in the proposal of a novel PHS, called Lyra2. This new algorithm preserves the flexibility of existing functions (including its predecessor, Lyra), but provides higher security. This step also involves the development of a refe- 
rence implementation for allowing validation of its viability and comparison with existing PHS solutions;

- Evaluation: comparison between the structures of the current PHSs and the Lyra2 in order to verify that, (1) by construction, Lyra2's security is higher than that provided by existing PHS, and (2) its performance is at least as good as that of alternative solutions;

- Thesis writing: creation of a thesis encompassing the obtained results and analyses.

These steps are rather sequential, with frequent iterations between them (e.g., performance measurements usually lead to improvements to the algorithm's design).

\subsection{Document Organization}

The rest of this document is organized as follows. Chapter 2 describes the basic notation and outlines the concept of hash functions, cryptographic sponges and password hashing schemes, describing the main requirements of theses algorithms. Chapter 3 discusses the related work. Chapter 4 introduces Lyra2's core and its design rationale, while Chapter 5 analyzes its security. Chapter 6 presents our benchmark results and comparisons with existing PHS. Finally, Chapter 7 encloses our concluding remarks, main results and plans for future work. 


\section{BACKGROUND}

For better understanding the concepts explored in this document, it is necessary to clearly understand some basic concepts involved in the area of Cryptography. This is the main goal of this chapter, which covers the basic services provided by hash functions and how they can be implemented using the concept of cryptographic sponges. In addition, it also summarizes the characteristics, utilization and main security aspects of Password Hashing Schemes (PHS), which are the focus of this research.

In what follows and throughout this document, we use the notation and symbols shown in the "List of Symbols" (page xv).

\section{$2.1 \quad$ Hash-Functions}

Let $H$ be a function, we call $H$ as Hash-Function if $H:\{0,1\}^{*} \mapsto\{0,1\}^{h}$, where $h \in \mathbb{N}$ (TERADA, 2008). In other words, a Hash-Function $H$ is a one-way and non-invertible transformation that maps an arbitrary-length input $x$ to a fixed $h$ length output $y=H(x)$, called the hash-value, or simply the hash, of $x$. Therefore, the hash can be seen as a "digest" of $x$.

Hash-functions can be used to verify the integrity of a message $x$ : since a modification in $x$ will result in a different hash, any user can verify that the modified $x$ does not map to $H(x)$ (SIMPLICIO JR, 2010). In this case, the integrity of $H(x)$, must be ensured somehow, or attackers could replace $x$ by $x^{\prime}$ 
at the same time that they replace $H(x)$ by $H\left(x^{\prime}\right)$, misleading the verification process.

We note that there is also a more generic definition for hash functions without the one-way requirement (MENEZES et al., 1996), but for the purposes of this document such alternative is not considered because password hashing schemes, which are the focus of the discussion, requires the a $H$ that is not easily invertible.

\subsubsection{Security}

Since hash-functions are "many-to-one" functions, the existence of collisions (pairs of inputs $x$ and $x^{\prime}$ that are mapped to the same output $y=H(x)$ ) is unavoidable. Indeed, supposing that all input messages have a length of at most $t$ bits, that the outputs are $h$-bit long and that all $2^{h}$ outputs are equiprobable, then $2^{t-h}$ inputs will map to each output, and two input picked at random will yield to the same output with a probability of $2^{-h}$. To prevent attackers from using this property to their advantage, secure hash algorithms must satisfy at least the following three requirements:

- First Pre-image Resistance: given a hash $y=H(x)$, it is computationally infeasible to find any $x$ having that hash-value, i.e., it is computationally infeasible to "invert" the hash-function

- Second Pre-image Resistance: given $x$ and its corresponding hash $y=H(x)$, it is computationally infeasible to "find any other input" $x^{\prime}$ that maps to the same hash, i.e., it is computationally infeasible find a $x^{\prime}$ such that $x^{\prime} \neq x$ and $y=H\left(x^{\prime}\right)=H(x)$.

- Collision Resistance: it is computationally infeasible to "find any two distinct inputs" $x$ and $x^{\prime}$ that map to the same hash-value, i.e., it is computationally infeasible to find $H(x)=H\left(x^{\prime}\right)$ where $x \neq x^{\prime}$. 


\section{$2.2 \quad$ Cryptographic Sponges}

The concept of cryptographic sponges was formally introduced by Bertoni et al. in (BERTONI et al., 2007) and is described in detail in (BERTONI et al., 2011a). The elegant design of sponges has also motivated the creation of more general structures, such as the Parazoa family of functions (ANDREEVA; MENNINK; PRENEEL, 2011). Indeed, their flexibility is probably among the reasons that led Keccak (BERTONI et al., 2011b), one of the members of the sponge family, to be elected as the new Secure Hash Algorithm (SHA-3).

\subsubsection{Basic Structure}

In a nutshell, differently from the hash functions, sponge functions provide an interesting way of building hash functions with arbitrary input and output lengths. Such functions are based on the so-called sponge construction, an iterated mode of operation that uses a fixed-length permutation (or transformation) $f$ and a padding rule pad. More specifically, and as depicted in Figure 1, sponge functions rely on an internal state of $w=b+c$ bits, initially set to zero, and operate on an (padded) input $M$ cut into $b$-bit blocks. This is done by iteratively applying $f$ to the sponge's internal state, operation interleaved with the entry of input bits (during the absorbing phase) or the subsequent retrieval of output bits

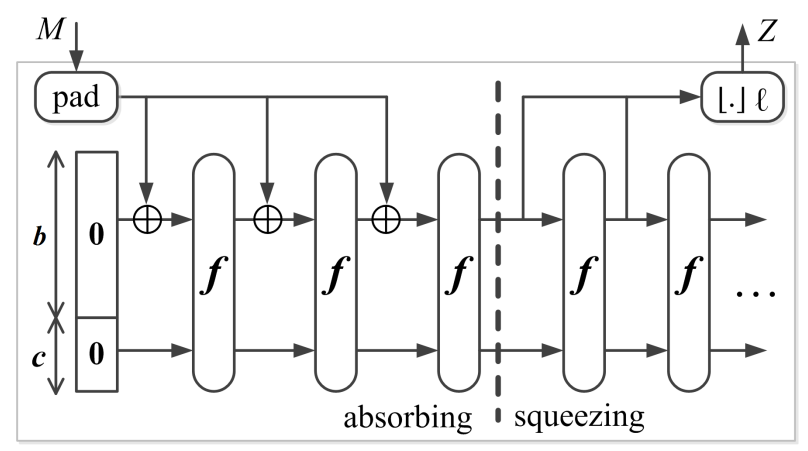

Figure 1: Overview of the sponge construction $Z=[f$, pad, $b](M, \ell)$. Adapted from (BERTONI et al., 2011a). 
(during the squeezing phase). The process stops when all input bits consumed in the absorbing phase are mapped into the resulting $\ell$-bit output string. Typically, the $f$ transformation is itself iterative, being parameterized by a number of rounds (e.g., 24 for Keccak operating with 64-bit words (BERTONI et al., 2011b)).

The sponge's internal state is, thus, composed by two parts: the $b$-bit long outer part, which interacts directly with the sponge's input, and the $c$-bit long inner part, which is only affected by the input by means of the $f$ transformation. The parameters $w, b$ and $c$ are called, respectively, the width, bitrate, and the capacity of the sponge.

\subsubsection{The duplex construction}

A similar structure derived from the sponge concept is the Duplex construction (BERTONI et al., 2011a), depicted in Figure 2.

Unlike regular sponges, which are stateless in between calls (i.e., calls between absorbing and squeezing phases do not depend of the inputs received), a duplex function is stateful: it takes a variable-length input string and provides a variablelength output that depends on all inputs received so far. In other words, although the internal state of a duplex function is filled with zeros upon initialization, it is stored after each call to the duplex object rather than repeatedly reset. In this

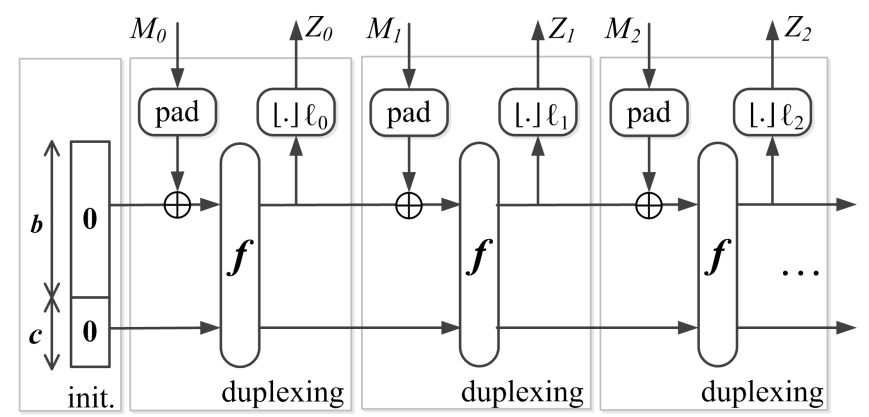

Figure 2: Overview of the duplex construction. Adapted from (BERTONI et al., 2011a). 
case, the input string $x$ must be short enough to fit in a single $b$-bit block after padding, and the output length $\ell$ must satisfy $\ell \leqslant b$.

\subsubsection{Security}

The fundamental attacks against cryptographic sponge functions which allow it to be distinguished from a random oracle are called primary attacks (BERTONI et al., 2011a). In order to be considered secure, a sponge function must resist to such attacks, which implies that the following operations must be computationally infeasible (BERTONI et al., 2011a):

- Finding a path (a sequence of bytes to be absorbed by the sponge) $P$ leading to a given internal state $s$.

- Finding two different paths leading to the same internal state $s$.

- Finding the internal state $s$ for a given output $Z$.

\subsection{Password Hashing Schemes (PHS)}

As previously discussed, the basic requirement for a PHS is to be noninvertible, so that recovering the password from its output is computationally infeasible. Moreover, a good PHS's output is expected to be indistinguishable from random bit strings, preventing an attacker from discarding part of the password space based on perceived patterns (KELSEY et al., 1998). In principle, those requirements can be easily accomplished simply by using a secure hash function, which by itself ensures that the best attack venue against the derived key is through brute force (possibly aided by a dictionary or "usual" password structures (NIST, 2011; WEIR et al., 2009)).

What any modern PHS does, then, is to include techniques that raise the 
cost of brute-force attacks. A first strategy for accomplishing this is to take as input not only the user-memorizable password pwd itself, but also a sequence of random bits known as salt. The presence of such random variable thwarts several attacks based on pre-built tables of common passwords, i.e., the attacker is forced to create a new table from scratch for every different salt (KALISKI, 2000; KELSEY et al., 1998). The salt can, thus, be seen as an index into a large set of possible keys derived from pwd, and needs not to be memorized or kept secret (KALISKI, 2000).

A second strategy is to purposely raise the cost of every password guess in terms of computational resources, such as processing time and/or memory usage. This certainly also raises the cost of authenticating a legitimate user entering the correct password, meaning that the algorithm needs to be configured so that the burden placed on the target platform is minimally noticeable by humans. Therefore, the legitimate users and their platforms are ultimately what imposes an upper limit on how computationally expensive the PHS can be for themselves and for attackers. For example, a human user running a single PHS instance is unlikely to consider a nuisance that the password hashing process takes $1 \mathrm{~s}$ to run and uses a small part of the machine's free memory, e.g., 20 MB. On the other hand, supposing that the password hashing process cannot be divided into smaller parallelizable tasks, achieving a throughput of 1,000 passwords tested per second requires $20 \mathrm{~GB}$ of memory and 1,000 processing units as powerful as that of the legitimate user.

A third strategy, especially useful when the PHS involves both processing time and memory usage, is to use a design with low parallelizability. The reasoning is as follows. For an attacker with access to $p$ processing cores, there is usually no difference between assigning one password guess to each core or parallelizing a single guess so it is processed $p$ times faster: in both scenarios, the total password 
guessing throughput is the same. However, a sequential design that involves configurable memory usage imposes an interesting penalty to attackers who do not have enough memory for running the $p$ guesses in parallel. For example, suppose that testing a guess involves $m$ bytes of memory and the execution of $n$ instructions. Suppose also that the attacker's device has $100 m$ bytes of memory and 1000 cores, and that each core executes $n$ instructions per second. In this scenario, up to 100 guesses can be tested per second against a strictly sequential algorithm (one per core), the other 900 cores remaining idle because they have no memory to run.

Aiming to provide a deeper understanding on the challenges faced by PHS solutions, we next discuss the main characteristics of platforms used by attackers and how existing solutions avoid those threats.

\subsubsection{Attack platforms}

The most dangerous threats faced by any PHS comes from platforms that benefit from "economies of scale", especially when cheap, massively parallel hardware is available. The most prominent examples of such platforms are Graphics Processing Units (GPUs) and custom hardware synthesized from FPGAs (DÜRMUTH; GÜNEYSU; KASPER, 2012).

\subsubsection{Graphics Processing Units (GPUs)}

Following the increasing demand for high-definition real-time rendering, Graphics Processing Units (GPUs) have traditionally carried a large number of processing cores, boosting its parallelization capability. Only more recently, however, GPUs evolved from specific platforms into devices for universal computation and started support standardized languages that help harness their computational power, such as CUDA (NVIDIA, 2014) and OpenCL (KHRONOS GROUP, 
2012)). As a result, they became more intensively employed for more general purposes, including password cracking (DÜRMUTH; GÜNEYSU; KASPER, 2012; SPRENGERS, 2011).

As modern GPUs include a few thousands processing cores in a single piece of equipment, the task of executing multiple threads in parallel becomes simple and cheap. They are, thus, ideal when the goal is to test multiple passwords independently or to parallelize a PHS's internal instructions. For example, NVidia's Tesla K20X, one of the top GPUs available, has a total of 2,688 processing cores operating at $732 \mathrm{MHz}$, as well as $6 \mathrm{~GB}$ of shared DRAM with a bandwidth of 250 GB per second (NVIDIA, 2012). Its computational power can also be further expanded by using the host machine's resources (NVIDIA, 2014), although this is also likely to limit the memory throughput. Supposing this GPU is used to attack a PHS whose parametrization makes it run in $1 \mathrm{~s}$ and take less than $2.23 \mathrm{MB}$ of memory, it is easy to conceive an implementation that tests 2,688 passwords per second. With a higher memory usage, however, this number is deemed to drop due to the GPU's memory limit of 6 GB. For example, if a sequential PHS requires $20 \mathrm{MB}$ of DRAM, the maximum number of cores that could be used simultaneously becomes 300 , only $11 \%$ of the total available.

\subsubsection{Field Programmable Gate Arrays (FPGAs)}

An FPGA is a collection of configurable logic blocks wired together and with memory elements, forming a programmable and high-performance integrated circuit. In addition, as such devices are configured to perform a specific task, they can be highly optimized for its purpose (e.g., using pipelining (DANDASS, 2008; KAKAROUNTAS et al., 2006)). Hence, as long as enough resources (i.e., logic gates and memory) are available in the underlying hardware, FPGAs potentially yield a more cost-effective solution than what would be achieved with a general- 
purpose CPU of similar cost (MARECHAL, 2008).

When compared to GPUs, FPGAs may also be advantageous due to the latter's considerably lower energy consumption (CHUNG et al., 2010; FOWERS et al., 2012), which can be further reduced if its circuit is synthesized in the form of custom logic hardware (ASIC) (CHUNG et al., 2010).

A recent example of password cracking using FPGAs is presented in (DÜRMUTH; GÜNEYSU; KASPER, 2012). Using a RIVYERA S3-5000 cluster (SCIENGINES, 2013a) with 128 FPGAs against PBKDF2-SHA-512, the authors reported a throughput of 356,352 passwords tested per second in an architecture having 5,376 password processed in parallel. It is interesting to notice that one of the reasons that made these results possible is the small memory usage of the PBKDF2 algorithm, as most of the underlying SHA-2 processing is performed using the device's memory cache (much faster than DRAM) (DÜRMUTH; GÜNEYSU; KASPER, 2012, Sec. 4.2). Against a PHS requiring 20 MB to run, for example, the resulting throughput would presumably be much lower, especially considering that the FPGAs employed can have up to 64 MB of DRAM (SCIENGINES, 2013a) and, thus, up to three passwords can be processed in parallel rather than 5,376 .

Interestingly, a PHS that requires a similar memory usage would be troublesome even for state-of-the-art clusters, such as the newer RIVYERA V7-2000T (SCIENGINES, 2013b). This powerful cluster carries up to four Xilinx Virtex-7 FPGAs and up to $128 \mathrm{~GB}$ of shared DRAM, in addition to the $20 \mathrm{~GB}$ available in each FPGA (SCIENGINES, 2013b). Despite being much more powerful, in principle it would still be unable to test more than 2,600 passwords in parallel against a PHS that strictly requires $20 \mathrm{MB}$ to run. 


\section{RELATED WORKS}

Following the call for candidates made by the Password Hashing Competition (PHC), several new Password Hashing Schemes have emerged in the last years. To be more specific, 24 new schemes were proposed, two of which voluntarily gave up the competition (PHC, 2013); later, out of the 22 remaining proposals, only 9 were selected for the final phase of the PHC (PHC, 2015c). In what follows, we describe the main password hashing solutions available in the literature and also give a brief overview of the PHC's finalists that, like Lyra2, allow both memory usage and processing time to be configured. For conciseness, however, we do not cover all details of each PHC algorithm, but only the main characteristics that are useful for the discussion. Nevertheless, we refer the interested reader to the PHC official website (PHC, 2013) for details on each submission to the competition.

\subsection{Pre-PHC Schemes}

Arguably, the main password hashing solutions available in the literature before the start of PHC were (PHC, 2013): PBKDF2 (KALISKI, 2000), bcrypt (PROVOS; MAZIÈRES, 1999), scrypt (PERCIVAL, 2009) and Lyra2's predecessor (simply called Lyra) (ALMEIDA et al., 2014). These schemes are described as follows. 


\subsubsection{PBKDF2}

The Password-Based Key Derivation Function version 2 (PBKDF2) algorithm (KALISKI, 2000) was originally proposed in 2000 as part of RSA Laboratories' Public-Key Cryptography Standards series, namely PKCS\#5. It is nowadays present in several security tools, such as TrueCrypt (TRUECRYPT, 2012), Apple's iOS for encrypting user passwords (Apple, 2012) and Android operating system for filesystem encryption, since version 3.0 (AOSP, 2012), and has been formally analyzed in several circumstances (YAO; YIN, 2005; BELLARE; RISTENPART; TESSARO, 2012).

Basically, PBKDF2 (see Algorithm 1) iteratively applies the underlying pseudorandom function $P R F$ to the concatenation of $p w d$ and a variable $U_{i}$, i.e., it makes $U_{i}=P R F\left(p w d, U_{i-1}\right)$ for each iteration $1 \leqslant i \leqslant T$. The initial value $U_{0}$ corresponds to the concatenation of the user-provided salt and a variable $l$, where $l$ corresponds to the number of required output blocks, and $h$ is the length of the pseudorandom function. The $l$-th block of the $k$-long key is then computed as $K_{l}=U_{1} \oplus U_{2} \oplus \ldots \oplus U_{T}$, where $k$ is the desired key length.

PBKDF2 allows users to control its total running time by configuring the $T$

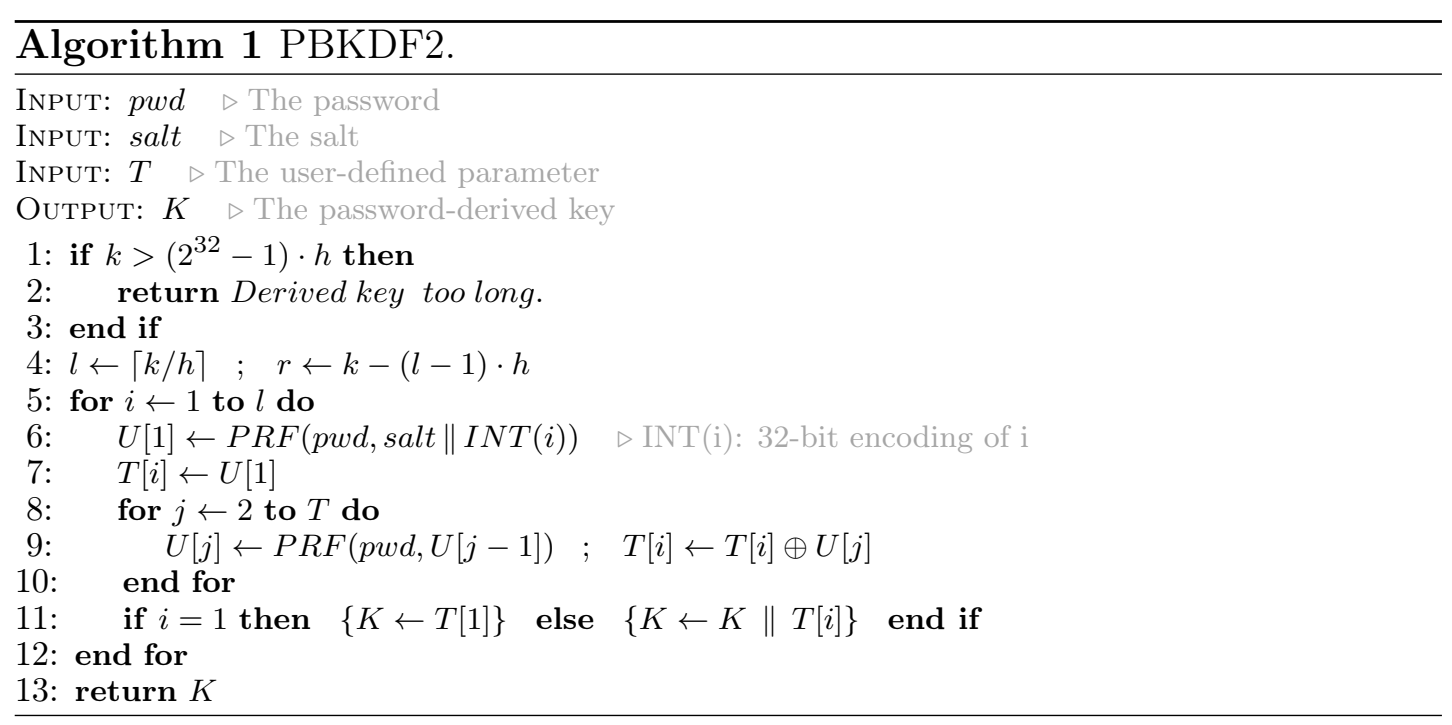


parameter. Since the password hashing process is strictly sequential (one cannot compute $U_{i}$ without first obtaining $\left.U_{i-1}\right)$, its internal structure is not parallelizable. However, as the amount of memory used by PBKDF2 is quite small, the cost of implementing brute force attacks against it by means of multiple processing units remains reasonably low.

\subsubsection{Bcrypt}

Another solution that allows users to configure the password hashing processing time is bcrypt (PROVOS; MAZIĖRES, 1999). The scheme is based on a customized version of the 64-bit cipher algorithm Blowfish (SCHNEIER, 1994), called EksBlowflish ("expensive key schedule blowfish").

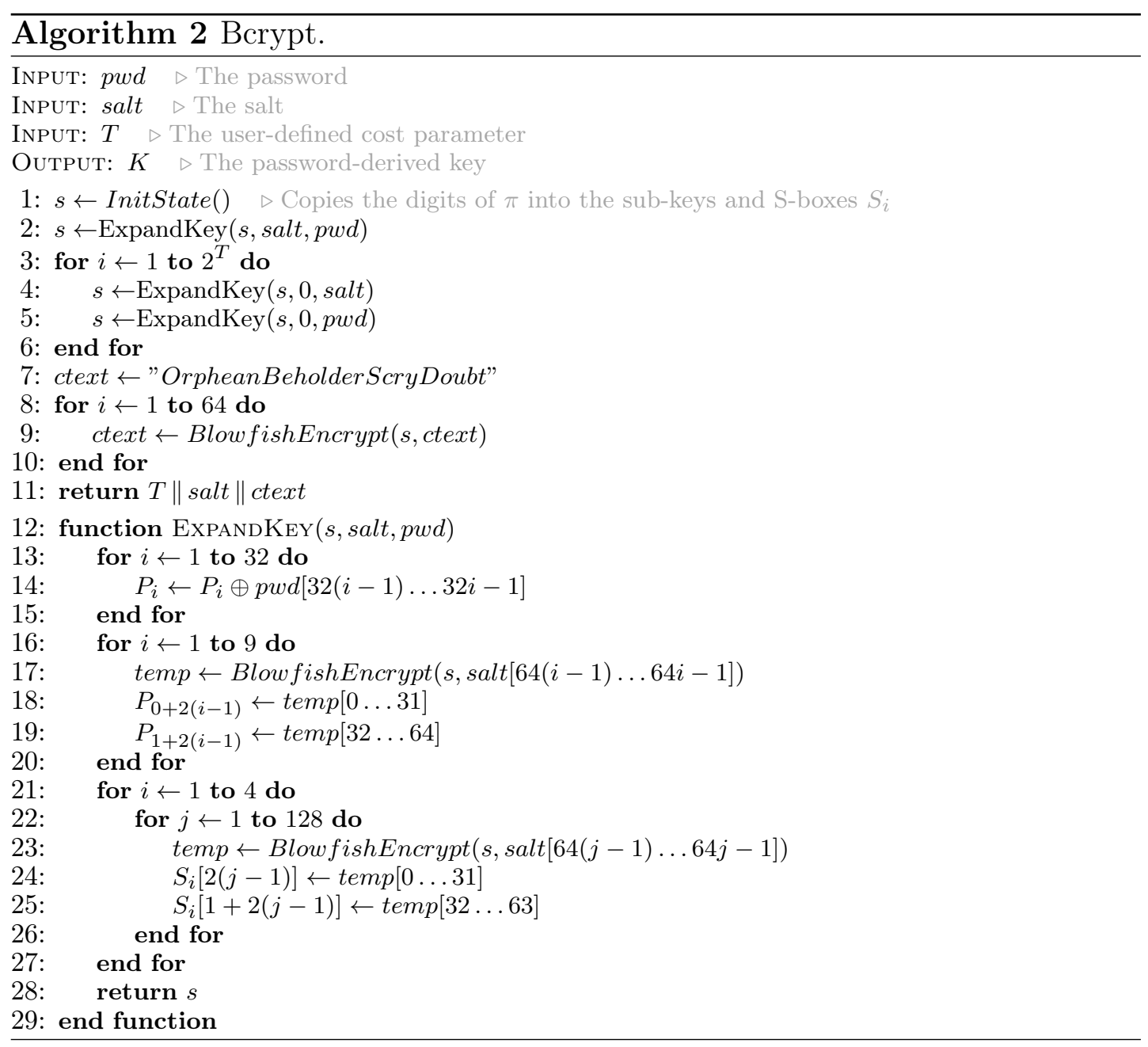


Both algorithms use the same encryption process, differing only on how they compute their subkeys and S-boxes. Bcrypt consists in initializing EksBlowfish's subkeys and S-Boxes with the salt and password, using the so-called EksBlowfishSetup function, and then using EksBlowfish for iteratively encrypting a constant string, 64 times.

EksBlowfishSetup starts by copying the first digits of the number $\pi$ into the subkeys and S-boxes $S_{i}$ (see Algorithm 2). Then, it updates the subkeys and Sboxes by invoking ExpandKey(salt,pwd), for a 128-bit salt value. Basically, this function (1) cyclically XORs the password with the current subkeys, and then (2) iteratively blowfish-encrypts one of the halves of the salt, the resulting ciphertext being XORed with the salt's other half and also replacing the next two subkeys (or S-Boxes, after all subkeys are replaced). For example, in the first iteration, the first 64 bits of the salt are encrypted, and then the result is XORed with its second half and replaces the first two subkeys; this new set of subkeys is used in the subsequent encryption.

After all subkeys and S-Boxes are updated, bcrypt alternately calls $\operatorname{ExpandKey}(0$, salt $)$ and then $\operatorname{ExpandKey}(0, p w d)$, for $2^{T}$ iterations. The userdefined parameter $T$ determines, thus, the time spent on this subkey and S-Box updating process, effectively controlling the algorithm's total processing time.

Like PBKDF2, bcrypt allows users to parameterize only its total running time, i.e., does not allow the users the amount of memory used by the algorithm, In addition to this shortcoming, some of its characteristics can be considered (small) disadvantages when compared with PBKDF2. First, bcrypt employs a dedicated structure (EksBlowfish) rather than a conventional hash function, leading to the need of implementing a whole new cryptographic primitive and, thus, raising the algorithm's code size. Second, EksBlowfishSetup's internal loop grows 
exponentially with the $T$ parameter, making it harder to fine-tune bcrypt's total execution time without a linearly growing external loop. Finally, bcrypt displays the unusual (albeit minor) restriction of being unable to handle passwords having more than 56 bytes. This latter issue is not a serious limitation, not only because larger passwords are unlikely to be "human-memorizable", but also because this could be overcome by pre-hashing the password to the required 56 bytes before the call to the bcrypt algorithm. Nonetheless, this does impairs the scheme's flexibility.

\subsubsection{Scrypt}

The design of scrypt (PERCIVAL, 2009) focuses on coupling memory and time costs. For this, scrypt employs the concept of "sequential memory-hard"

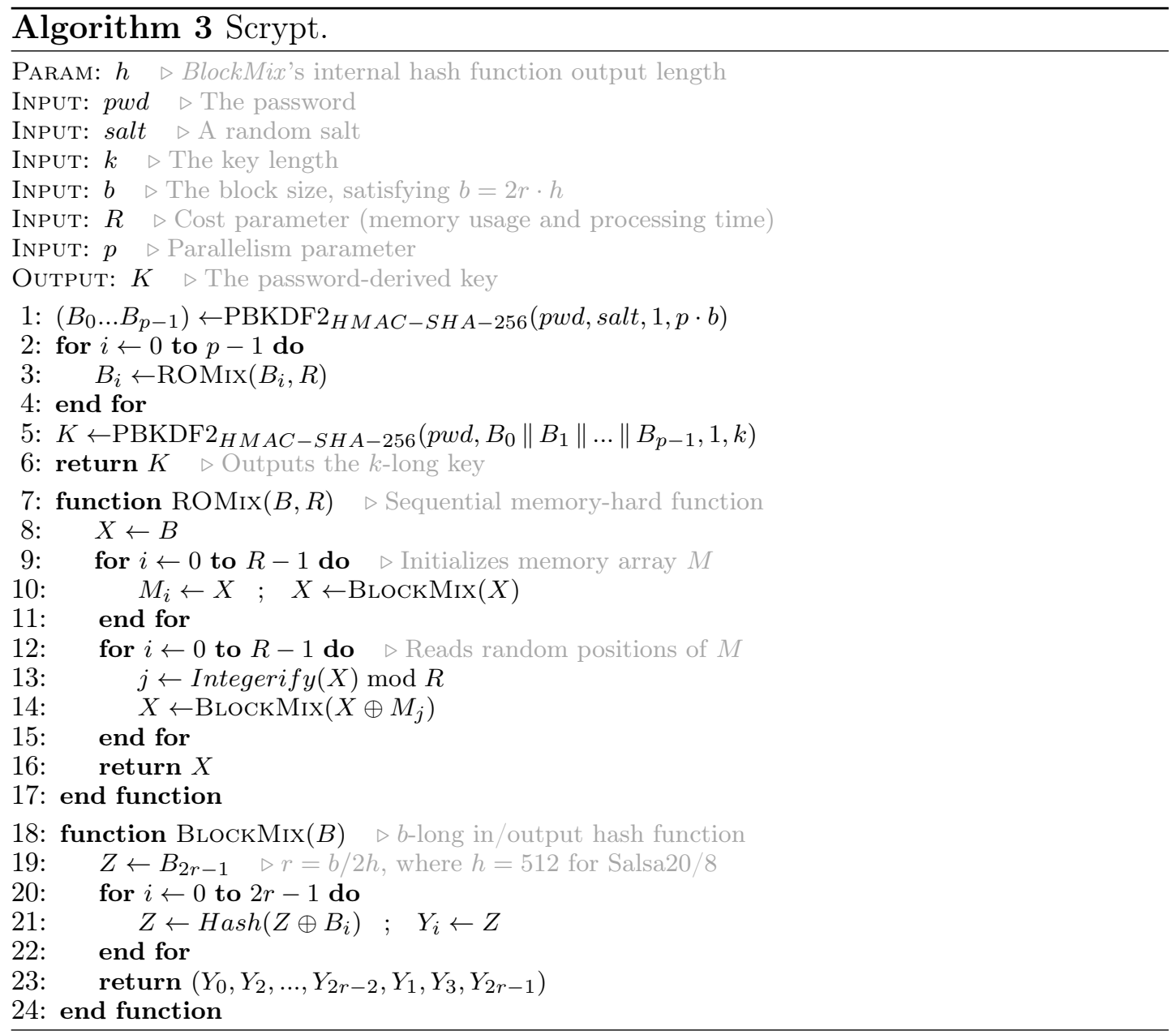


functions: an algorithm that asymptotically uses almost as much memory as it requires operations and for which a parallel implementation cannot asymptotically obtain a significantly lower cost. Informally, this means that if the number of operations and the amount of memory used in the regular operation of the algorithm are both $O(R)$, where $R$ is a system parameter, then any attack trying to exploit time-memory trade-offs (TMTO) should always lead to a $\Omega\left(R^{2}\right)$ timememory product, limiting the attacker's capability of using strategies for reducing the algorithm's total memory usage. For example, the complexity of a memoryfree attack - i.e., an attack for which the memory usage is reduced to $O(1)-$ becomes $\Omega\left(R^{2}\right)$, which should compel attackers to use more memory. For conciseness, we refer the reader to (PERCIVAL, 2009) for a more formal definition of the memory-hardness concept.

The following steps compose scrypt's operation (see Algorithm 3). First, it initializes $p$-long memory blocks $B_{i}$. This is done using the PBKDF2 algorithm with HMAC-SHA-256 (NIST, 2002b) as an underlying hash function and a single iteration. Then, each $B_{i}$ is processed (incrementally or in parallel) by the sequential memory-hard ROMix function. Basically, ROMix initializes an array $M$ of $R b$-long elements by iteratively hashing $B_{i}$. It then visits $R$ positions of $M$ at random, updating the internal state variable $X$ during this (strictly sequential) process in order to ascertain that those positions are indeed available in memory.

The hash function employed by ROMix is called BlockMix, which emulates a function having arbitrary ( $b$-long) input and output lengths; this is done using the Salsa20/8 (BERNSTEIN, 2008) stream cipher, whose output length is $h=512$. After the $p$ ROMix processes are over, the $B_{i}$ blocks are used as salt in one final iteration of the PBKDF2 algorithm, outputting key $K$.

Scrypt displays a very interesting design, being one of the few existing solutions that allow the configuration of both processing and memory costs. One 
of its main shortcomings is probably the fact that it strongly couples memory and processing requirements for a legitimate user. Specifically, scrypt's design prevents users from raising the algorithm's processing time while maintaining a fixed amount of memory usage, unless they are willing to raise the $p$ parameter and allow further parallelism to be exploited by attackers.

Another inconvenience with scrypt is the fact that it employs two different underlying hash functions, HMAC-SHA-256 (for the PBKDF2 algorithm) and Salsa20/8 (as the core of the BlockMix function), leading to increased implementation complexity.

Finally, even though Salsa20/8's known vulnerabilities (AUMASSON et al., 2008) are not expected to put the security of scrypt in hazard (PERCIVAL, 2009), using a stronger alternative would be at least advisable, especially considering that the scheme's structure does not impose serious restrictions on the internal hash algorithm used by BlockMix. In this case, a sponge function could itself be an alternative, with the advantage that, since sponges support inputs and outputs of any length, the whole BlockMix structure could be replaced. However, sponges' intrinsic properties make some of scrypt's operations unnecessary: for example, since sponges support inputs and outputs of any length, the whole BlockMix structure could be replaced.

\subsubsection{Lyra}

Inspired by scrypt's design, Lyra (ALMEIDA et al., 2014) builds on the properties of sponges to provide not only a simpler, but also more secure solution. Indeed, Lyra stays on the "strong" side of the memory-hardness concept: the processing cost of attacks involving less memory than specified by the algorithm grows much faster than quadratically, surpassing the best achievable with scrypt and thwarting the exploitation of time-memory trade-offs (TMTO). This charac- 
teristic should discourage attackers from trading memory usage for processing time, which is exactly the goal of a PHS in which the usage of both resources are configurable.

Lyra's steps as described in (ALMEIDA et al., 2014) are detailed in Algorithm 4. Basically, Lyra builds upon (reduced-round) operations of a cryptographic sponge for (1) building a memory matrix, (2) visiting its rows in a pseudorandom fashion, as many times as defined by the user, and then (3) providing the desired number of bits as output. More precisely, the first part of the algorithm, called the Setup Phase (lines $1-8$ ), comprises the construction of a $R \times C$ memory matrix whose cells are $b$-long blocks, where $R$ and $C$ are user-defined parameters and $b$ is the underlying sponge's bitrate (in bits). Without resetting the sponge's internal state, the algorithm enters then the Wandering Phase (lines 9-19), in which $(T \cdot R)$ rows are visited in a pseudorandom fashion, aiming to ensure that the whole memory matrix is still available in memory. Every row visited in this manner has all of its cells read and combined with the output of the underlying

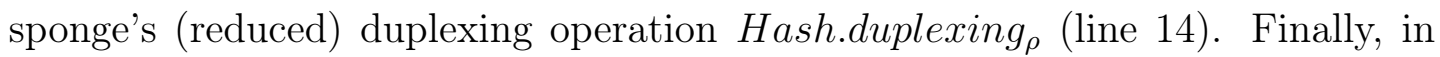
the Wrap-up Phase (lines 20 - 22), the final key is computed by first absorbing the salt one last time and then squeezing the (full-round) sponge, once again using its current internal state. The stateful, full-round sponge employed in this last stage ensures that the whole process is both non-invertible and of sequential nature.

While Lyra2 also builds upon reduced-round sponges for achieving high performance, it also addresses some shortcomings of Lyra's design. First, Lyra's Setup is quite simple, each iteration of its loop (lines 8 to 4) duplexing only the row that was computed in the previous iteration. As a result, the Setup can be executed with a cost of $R \cdot \sigma$ while keeping in memory a single row of the memory matrix. Second, Lyra's duplexing operations performed during the 


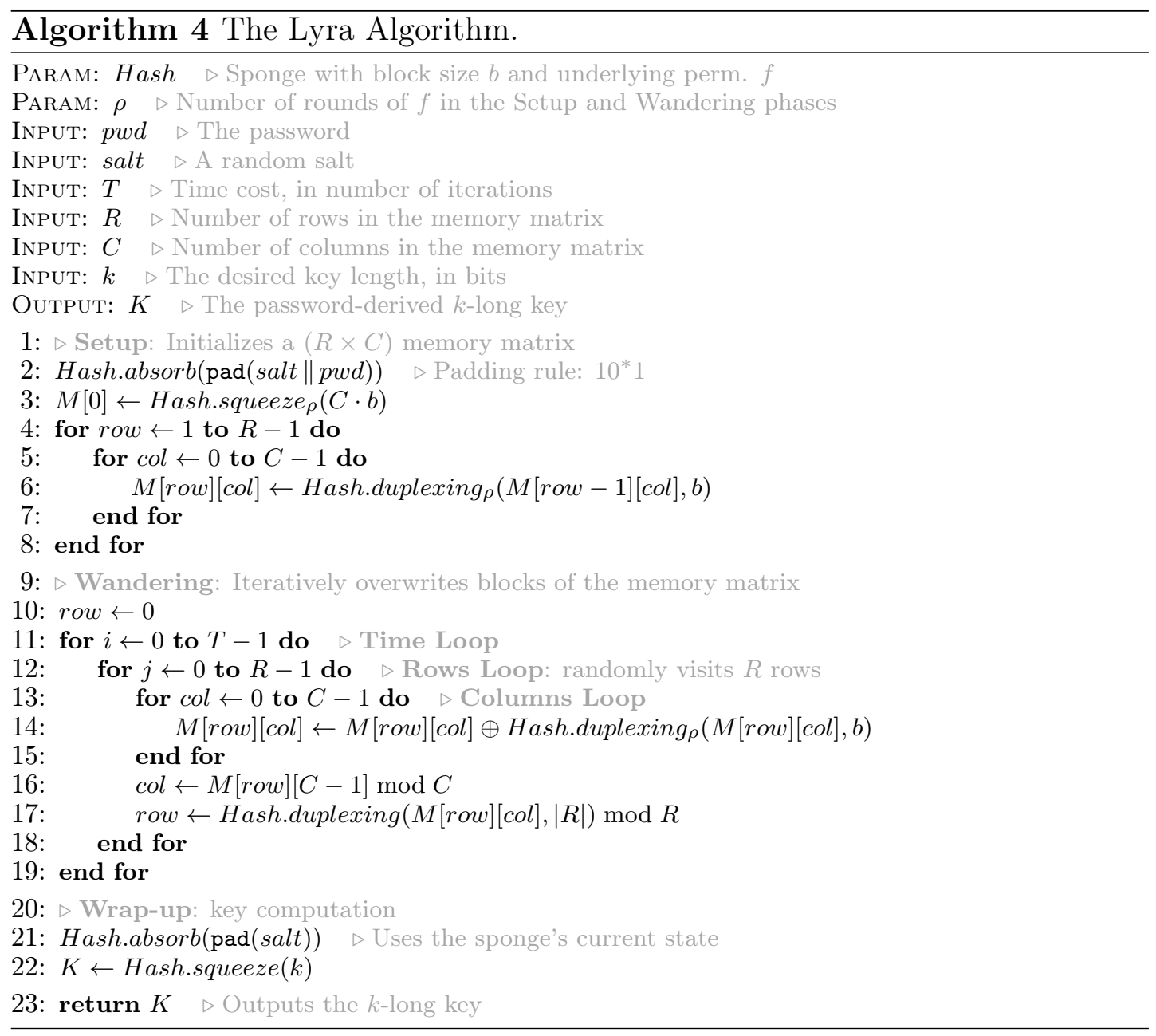

Wandering phase involve only one pseudorandomly-picked row, which is read and written upon. As it turns out, one can add extra rows to this process with little impact on performance on modern platforms, as existing memory devices have enough bandwidth to support a higher number of memory reads/writes. Raising the amount of memory accesses also has positive results on security, for two reasons: (1) if an attacker tries to trade memory usage for extra processing, a potentially larger number of rows will have to be recomputed for performing each duplexing operation; and (2) attackers trying to run multiple instances of the password hashing algorithm (or recomputations in an attack exploiting time-memory trade-offs) will need to account for such increased bandwidth usage. Lyra2's design addresses both of these issues, besides introducing a few other improvements (e.g., resistance to side-channel attacks). 


\subsection{Schemes from PHC}

The Password Hashing Competition (PHC) was created aiming to evaluate novel PHS designs and ideas in terms of security, performance and flexibility (PHC, 2013). In its first round, 22 candidates were submited to the competition: AntCrypt, Argon, battcrypt, Catena, Centrifuge, EARWORM, Gambit, Lanarea, Lyra2, Makwa, MCS_PHS, Omega Crypt, Parallel, PolyPassHash, POMELO, Pufferfish, RIG, Schvrch, Tortuga, TwoCats, Yarn and yescrypt. Besides these, two other schemes (Catfish and M3lcrypt) were submitted, but withdrew before the initial evaluation process.

After the first round evaluation, 9 finalists were announced as potential winners of the competition, 6 of which are memory-hard algorithms (PHC, 2015c): Argon, battcrypt, Catena, POMELO, yescrypt and Lyra2. This selection was based on many criteria (PHC, 2015c): defense against GPU/FPGA/ASIC attackers; defense against time-memory trade-offs; defense against side-channel leaks; defense against cryptanalytic attacks; elegance and simplicity of design; quality of the documentation; quality of the reference implementation; general soundness and simplicity of the algorithm; and originality and innovation. These criteria have no particular order, and the panel also took into account some specific applications, such as web-service authentication, client login, key derivation, or usage in embedded devices, presented by some candidates.

In the specific case of Lyra2, the algorithm was announced as a finalist due to its elegant sponge-based design, with a single external primitive, and its detailed security analysis (PHC, 2015c). At the end of the competition, this property, together with the approach adopted to side-channel resistance that also takes into account slow-memory attacks, led to a "special recognition" for Lyra2 (PHC, 2015b). On this occasion, Argon2 (which was not among the original candida- 
tes, but was accepted in the second round as a "new candidate" nevertheless) was announced as the PHC winner, and other three candidates received a special recognition; namely: Catena, for its agile framework approach and side-channel resistance; Makwa, for its unique delegation feature and its factoring-based security; and yescrypt, for its rich feature set and easy upgrade path from scrypt (PHC, 2015b).

In what follows, we briefly describe the other memory-hard finalists. For conciseness, however we do not analyze every detail of the algorithms, but only the main aspects that allow the reader to grasp the reason behind the numbers obtained in the comparative performance assessment presented later in Section 6.1.

\subsubsection{Argon2}

The Argon2 scheme was announced as an evolution of its predecessor, Argon. Although these algorithms have nothing in common, the PHC panel decided to accept Argon2 in the last phase of the competition after internal discussions and consultation to the other teams participating in the PHC, including the Lyra2 team (SIMPLICIO JR, 2015).

Contrasting with all other finalists, Argon2 displays more than one mode of operation which means that it works in a distinct way depending on its parameters. Namely, Argon2d's memory access pattern depends on the user's password, while Argon2i adopts a password-independent memory access (BIRYUKOV; DINU; KHOVRATOVICH, 2016); as a result, Argon2i displays high resistance against side-channel attacks, while Argon2d focuses on resistance against slow-memory attacks. Besides these two operation modes, the authors also provide a hybrid operation in its official repository, called Argon2id (PHC, 2015a), which was designed after the end of the PHC as a recommended tweak for the algorithm. This mode combines a password-independent memory access pattern 
when it fills the memory at the beginning of the algorithm's execution, and then revisits the memory in a password-dependent manner in the remainder of the process, which is actually very similar to what is done in Lyra2. Although the multiple modes of operation may actually be confusing to users, since even specialists do not always agree on how much side-channel vs. slow-memory resistance is necessary in a given practical scenario, this approach leads to a quite flexible design.

Another characteristic of the Argon2 scheme that shows that Lyra2 contributed to its final design is that it adopts as underlying cryptographic function the BlaMka multiplication-hardened sponge (BIRYUKOV; DINU; KHOVRATOVICH, 2016, Appendix A), which is actually one of the original results of this thesis, presented in Section 4.4.1

Like Lyra2 (and also Lyra), Argon2 was designed to thwart attacks involving Time-Memory trade-offs (TMTO), imposing large processing penalties to attackers who try to run the algorithm with less memory than a legitimate user. According to the security analysis presented by Argon2's authors, for a memory reduction of approximately $1 / 6$, the penalty should be approximately $(279601 \cdot T \cdot R) / 6 \approx 2^{15.5} \cdot T \cdot R$ for Argon2i and $(4753217 \cdot T \cdot R) / 6 \approx 2^{19.6} \cdot T \cdot R$ for Argon2d - where $T$ is the algorithm's time parameter and $R$ is its memory parameter - (BIRYUKOV; DINU; KHOVRATOVICH, 2016).

Argon2 can also be configured to use any amount of memory (e.g., it does not impose that the memory sizes must be a power of two, a limitation that appears in some other candidates). Nonetheless, the memory parameter should be larger than $8 p$ and a multiple of $4 p$, where $p$ is its degree of parallelism. 


\subsection{2 battcrypt}

Battcrypt (Blowfish All The Things [crypt]) is a simplified scrypt and targets server-side application (THOMAS, 2014). Internally, it uses the Blowfish block cipher (SCHNEIER, 1994) in the CBC mode of operation (NIST, 2001b), as well as the hash function SHA-512 (NIST, 2002a). According to Battcrypt's authors, Blowfish is used because it is well-studied and included in PHP implementations (THOMAS, 2014).

Despite its simple design, Battcrypt does not have an in-depth security analysis. Namely, Battcrypt's authors provide only a discussion on the scheme's security in terms of the underlying Blowfish primitive, concluding that, if the latter is broken, the same applies to battcrypt too. There is, however, no security analysis concerning its resistance to TMTO attacks, besides the complete absence of mechanisms for protecting the algorithm against side-channel attacks.

Another shortcoming of Battcrypt is that its memory usage cannot be easily fine-tuned, as its running time depends on the time parameter $T$ using a quite convoluted equation, namely $2^{\lfloor T / 2\rfloor \cdot((T \bmod 2)+2)}$ (THOMAS, 2014).

\subsubsection{Catena}

Catena was designed with a specific goal in mind: provide a memory-hard PHS with high resistance against side-channel attacks. To accomplish this goal, the algorithm avoids any password-dependent code branching, meaning that the order in which its internal memory is initialized and visited is completely deterministic, instead of pseudo-random. Specifically, this protects Catena against the so-called cache-timing attacks, in which the access to cache memory is monitored and used in the recovery of secret information (FORLER; LUCKS; WENZEL, 2013; BERNSTEIN, 2005). 
On the positive side, Catena displays a quite simple and elegant design, which makes it easy to understand and implement. Its authors also provide a quite complete security analysis, relying on the fact that Catena's structure is based on a special type of graph called "Bit-Reversal Graph" (LENGAUER; TARJAN, 1982). This particular graph type allows the security of Catena to be formally demonstrated (at least in part) using the pebble-game theory (COOK, 1973; DWORK; NAOR; WEE, 2005), allowing time-memory trade-offs (TMTO) against the algorithm to be tightly calculated. Specifically, according to Catena's analysis in (FORLER; LUCKS; WENZEL, 2013; FORLER; LUCKS; WENZEL, 2014) ${ }^{1}$ : the complexity of a memory-free attack against Catena is conjectured to be $\Theta\left(R^{T+1}\right)$; in attacks where the attacker choose the amount of memory to be used, the complexity is conjectured as $\Theta\left(R^{T+1} / M^{T}\right)$, where $R$ denotes the total memory used by legitimate users and $M$ the memory used by the attacker.

On the negative side, Catena does not allow a flexible choice of parameters, as the memory size must always be a power of two. In addition, the algorithm as originally presented to the PHC was quite slow, although in its last version it also allows the usage of a reduced-round sponge as proposed in Lyra (a feature also incorporated in Lyra2). Finally, and as further discussed in Section 5.3, providing full resistance against cache-timing attacks facilitates other types of attacks that can benefit from a purely deterministic memory visitation pattern. Therefore, by focusing on one (not necessarily most probable) attack venue, Catena ends up failing to provide a compromise between the different attack strategies at the disposal of password crackers.

Despite these shortcomings, Catena has the merit of bringing several interesting ideas concerning the usage of a PHS. Probably the most useful one is the concept of "server relief protocol", which allows a remote authentication server

\footnotetext{
${ }^{1}$ Note: to standardize the notation hereby employed, here we interpret Catena's garlic as $R$ and its depth as $T$
} 
to offload (most of) the computational effort involved in the password hashing process to the client (FORLER; LUCKS; WENZEL, 2014), leading to a more scalable authentication system. Another interesting idea is the concept of clientindependent update, a feature that allows the defender to increase the PHS's security parameters at any time, even for inactive accounts (FORLER; LUCKS; WENZEL, 2014), by re-hashing values already stored at the server's database. We note, however, that while these features are very relevant and described in detail for Catena, they can also be easily incorporated into any PHS (FORLER; LUCKS; WENZEL, 2013).

\subsubsection{POMELO}

POMELO has a quite simple design and, thus, is easy to implement (WU, 2015). Interestingly, this PHS does not adopt any existing cryptographic function as underlying primitive, but operates on 8-byte words and uses three state update functions developed specifically for this scheme. The first function is a simple nonlinear feedback function and the other two provide simple random memory access over data (HATZIVASILIS; PAPAEFSTATHIOU; MANIFAVAS, 2015).

According to POMELO's author, these tree functions protect POMELO against pre-image attacks (i.e., attempts to invert the hash for obtaining the password) and also TMTO attempts (WU, 2015). In addition, and similarly to what is done in Lyra2, POMELO's design is such that it provides a compromise between resistance against cache-timing attacks and to other attacks that might take advantage of purely deterministic memory visitation patterns. We note, however, that even though the scheme's authors provide a quite complete security analysis in its specification manual, the underlying POMELO functions have been target of criticism for not having a formal proof of their security (PHC, 2015d). 
Besides these potential doubts on the security of its primitives, POMELO also has one unusual security claim: protection against what the authors call "SIMD attacks", i.e., attacks that take advantage of SIMD instructions in modern platforms. Since most computer platforms do have support to SIMD, this is rather a limitation for legitimate users, who cannot take full advantage of available commodity hardware, than for attackers, who can build their own hardware platforms with whichever optimization they might find. In addition, the authors claim that POMELO is resistant to GPU and dedicated hardware attacks (WU, 2015). Finally, POMELO's design makes it difficult to fine tune its memory usage and processing time, as both grow exponentially with its $T$ and $R$ parameters.

\subsection{5 yescrypt}

The yescrypt scheme is strongly based on scrypt and, as the latter, allows legitimate users to adjust its memory and time costs to desired levels, using the $R$ and $T$ parameters. However, and unlike most PHC candidates, it provides a wide range of parameters besides $R$ and $T$, including the ability to indicate: whether it should be only read from memory after initializing it; if it should read from an additional, read-only memory device in addition to its internal memory; if it should operate in a scrypt-compatible mode; among many others. Furthermore, yescrypt uses many cryptographic primitives in its design, namely: SHA-256 (NIST, 2002a), HMAC (NIST, 2002b), Salsa20/8 (BERNSTEIN, 2008), and PBKDF2 (KALISKI, 2000) itself. This adds a lot of complexity to yescrypt's design, making it very hard to understand and, thus, analyze.

Indeed, its authors do not provide an in-depth security analysis about the algorithm, but rather high level claims about how its design strategies should thwart TMTO attempts as well as attacks using GPUs and dedicated hardware. The algorithm also has no mechanisms for protecting against side channel attacks, 
as all memory visitations are password-dependent, and does not allow the memory usage to be fine tuned, as the scheme requires its $R$ parameter to be a power of two (PESLYAK, 2015).

Despite its complexity, probably one of the main contributions of yescrypt's design is the introduction of the "multiplication-hardening" concept (COX, 2014; PESLYAK, 2015), which translates to the adoption of integer multiplications among the PHS's internal operations as a way to protect against attacks based on dedicated hardware. The reason is that, as verified in several benchmarks available in the literature (SHAND; BERTIN; VUILLEMIN, 1990; SODERQUIST; LEESER, 1995), the performance gain offered by hardware implementations of the multiplication operation is not much higher than what is obtained with software implementations running on commodity x86 platforms, for which such operations are already heavily optimized. Those optimizations appear in different levels, including compilers, advanced instruction sets (e.g., MMX, SSE and AVX), and architectural details of modern CPUs that resemble those of dedicated FPGAs. With these observations in mind, yescrypt iteratively performs several multiplications for each memory position it visits, and then mixes the result using one round of Salsa20/8. 


\section{$4 \quad$ LYRA2}

As any PHS, Lyra2 takes as input a salt and a password, creating a pseudorandom output that can then be used as key material for cryptographic algorithms or as an authentication string (NIST, 2009). Internally, the scheme's memory is organized as a matrix that is expected to remain in memory during the whole password hashing process: since its cells are iteratively read and written, discarding a cell for saving memory leads to the need of recomputing it whenever it is accessed once again, until the point it was last modified. The construction and visitation of the matrix is done using a stateful combination of the absorbing, squeezing and duplexing operations of the underlying sponge (i.e., its internal state is never reset to zero), ensuring the sequential nature of the whole process. Also, the number of times the matrix's cells are revisited after initialization is defined by the user, allowing Lyra2's execution time to be fine-tuned according to the target platform's resources.

In this chapter, we describe the core of the Lyra2 algorithm in detail and discuss its design rationale and resulting properties. Later, in Appendix B, we discuss a possible variant of the algorithm that may be useful in a different scenario. 


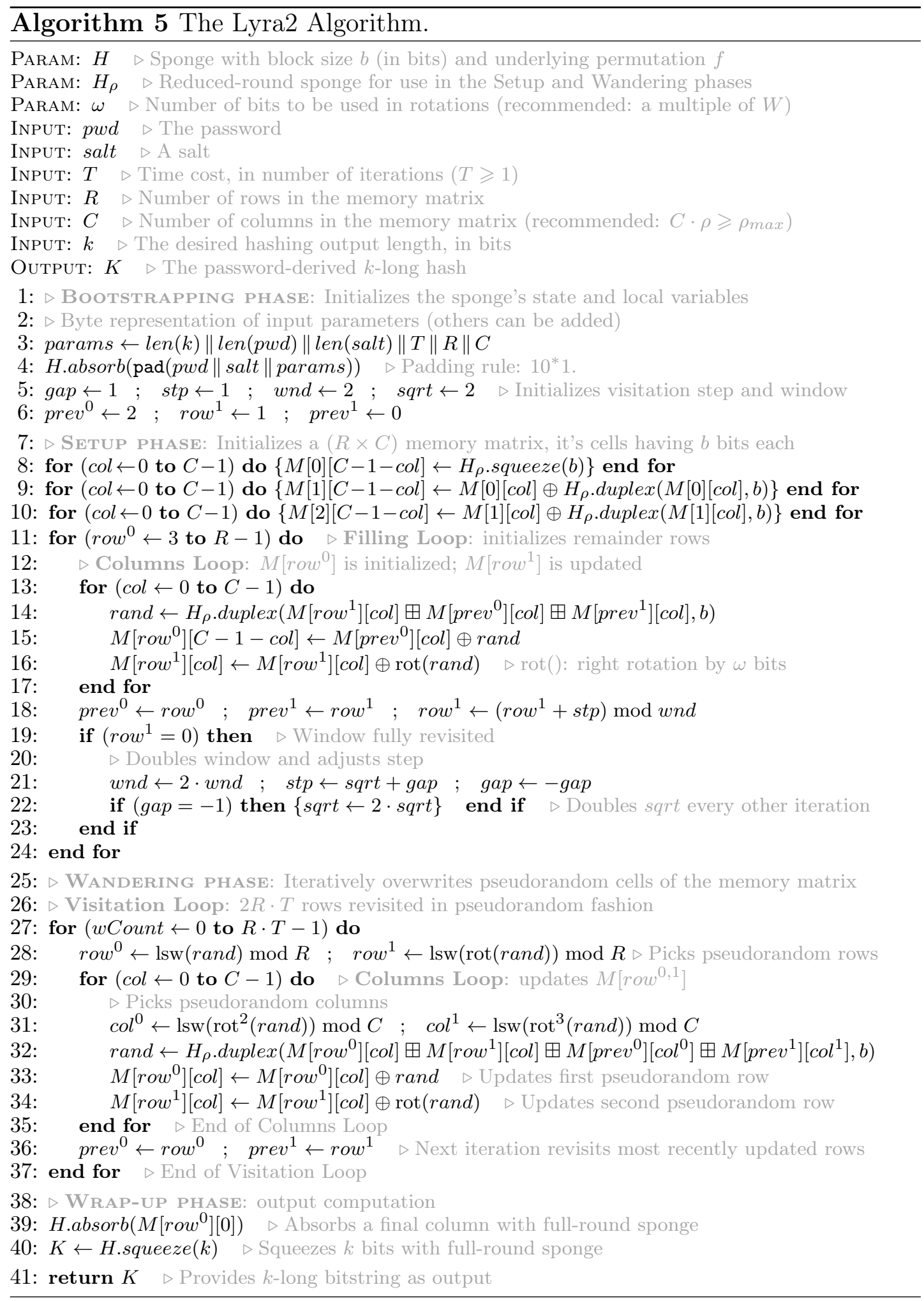




\subsection{Structure and rationale}

Lyra2's steps are shown in Algorithm 5. As highlighted in the pseudocode's comments, its operation is composed by four sequential phases: Bootstrapping, Setup, Wandering and Wrap-up. Along the description, we assume that all operations are performed using little-endian convention; they should, thus, be adapted accordingly for big-endian architectures (this basically applies to the rot operation).

\subsubsection{Bootstrapping}

The very first part of Lyra2 comprises the Bootstrapping of the algorithm's sponge and internal variables (lines 1 to 6 ). The set of variables $\{$ gap, stp, wnd, sqrt, $\operatorname{prev}^{0}$, row $^{1}$, prev $\left.^{1}\right\}$ initialized in lines 5 and 6 are useful only for the next stage of the algorithm, the Setup phase, so the discussion on their properties is left to Section 4.1.2.

Lyra2's sponge is initialized by absorbing the (properly padded) password and salt, together with a params bitstring, initializing a salt- and pwd-dependent state (line 4$)$. The padding rule adopted by Lyra2 is the multi-rate padding pad10*1 described in (BERTONI et al., 2011a), hereby denoted simply pad. This padding strategy appends a single bit 1 followed by as many bits 0 as necessary followed by a single bit 1 , so that at least 2 bits are appended. Since the password itself is not used in any other part of the algorithm, it can be discarded (e.g., overwritten with zeros) after this point.

In this first absorb operation, the goal of the params bitstring is basically to avoid collisions using trivial combinations of salts and passwords: for example, for any $(u, v \mid u+v=\alpha)$, we have a collision if $p w d=0^{u}$, salt $=0^{v}$ and params is an empty string; however, this should not occur if params explicitly includes 
$u$ and $v$. Therefore, params can be seen as an "extension" of the salt, including any amount of additional information, such as: the list of parameters passed to the PHS (including the lengths of the salt, password, and output); a user identification string; a domain name toward which the user is authenticating him/herself (useful in remote authentication scenarios); among others.

\subsubsection{The Setup phase}

Once the internal state of the sponge is initialized, Lyra2 enters the Setup Phase (lines 7 to 24). This phase comprises the construction of a $R \times C$ memory matrix whose cells are $b$-long blocks, where $R$ and $C$ are user-defined parameters and $b$ is the underlying sponge's bitrate (in bits).

For better performance when dealing with a potentially large memory matrix, the Setup relies on a "reduced-round sponge", i.e., the sponge's operations are performed with a reduced-round version of $f$, denoted $f_{\rho}$ for indicating that $\rho$ rounds are executed rather than the regular number of rounds $\rho_{\max }$. The advantage of using a reduced-round $f$ is that this approach accelerates the sponge's operations and, thus, it allows more memory positions to be covered than with the application of a full-round $f$ in a same amount of time. The adoption of reduced-round primitives in the core of cryptographic constructions is not unheard in the literature, as it is the main idea behind the ALRED family of message authentication algorithms (DAEMEN; RIJMEN, 2005; DAEMEN; RIJMEN, 2010; SIMPLICIO JR et al., 2009; SIMPLICIO JR; BARRETO, 2012). As further discussed in Section 4.2, even though the requirements in the context of password hashing are different, this strategy does not decrease the security of the scheme as long as $f_{\rho}$ is non-cyclic and highly non-linear, which should be the case for the vast majority of secure hash functions. In some scenarios, it may even be interesting to use a different function as $f_{\rho}$ rather than a reduced-round version of $f$ itself 
to attain higher speeds, which is possible as long the alternative satisfies the above-mentioned properties.

Except for rows $M[0]$ to $M[2]$, the sponge's reduced duplexing operation $H_{\rho}$.duplex is always called over the wordwise addition of three rows (line 14), all of which must be available in memory for the algorithm to proceed (see the Filling Loop, in lines 11-24).

- $M\left[\right.$ prev $\left.^{0}\right]$ : the last row ever initialized in any iteration of the Filling Loop, which means simply that $\operatorname{prev}^{0}=$ row $^{0}-1$;

- $M\left[\right.$ row $\left.^{1}\right]$ : a row that has been previously initialized and is now revisited; and

- $M\left[\right.$ prev $\left.^{1}\right]$ : the last row ever revisited (i.e., the most recently row indexed by row $\left.^{1}\right)$.

Given the short time between the computation and usage of $M\left[\right.$ prev $\left.^{0}\right]$ and $M\left[\right.$ prev $\left.^{1}\right]$, accessing them in a regular execution of Lyra 2 should not be a huge burden since both are likely to remain in cache. The same convenience does not apply to $M\left[\right.$ row $\left.^{1}\right]$, though, since it is picked from a window comprising rows initialized prior to $M\left[\right.$ prev $\left.^{0}\right]$. Therefore, this design takes advantage of caching while penalizing attacks in which a given $M\left[r_{0} w^{0}\right]$ is directly recomputed from the corresponding inputs: in this case, $M\left[\right.$ prev $\left.^{0}\right]$ and $M\left[\right.$ prev $\left.^{1}\right]$ may not be in cache, so all three rows must come from the main memory, raising memory latency and bandwidth. A similar effect could be achieved if the rows provided as the sponge's input were concatenated, but adding them together instead is advantageous because then the duplexing operation involves a single call to the underlying (reduced-round) $f$ rather than three.

After the reduced duplexing operation is performed, the resulting output (rand) affects two rows (lines 15 and 16): $M\left[r^{0} w^{0}\right]$, which has not been initialized 
yet, receives the values of $r$ and XORed with $M\left[\right.$ prev $\left.^{0}\right]$; meanwhile, the columns of the already initialized row $M\left[\right.$ row $\left.^{1}\right]$ have their values updated after being XORed with $\operatorname{rot}($ rand $)$, i.e., rand rotated to the right by $\omega$ bits. More formally, for $\omega=W$ and representing rand as an array of words $\operatorname{rand}[0] \ldots \operatorname{rand}[b / W-1]$ (i.e., the first $b$ bits of the outer state, from top to bottom as depicted in Figures 1 and 2), we have that $M\left[\right.$ row $\left.^{0}\right][C-1-i] \leftarrow M\left[\operatorname{prev}^{0}\right][i] \oplus \operatorname{rand}[i]$ and $M\left[\right.$ row $\left.^{1}\right][i] \leftarrow$ $M\left[\operatorname{row}^{1}\right][i] \oplus \operatorname{rand}[(i-1) \bmod (b / W)](0 \leqslant i \leqslant b / W-1)$. We notice that the rows are written from the highest to the lowest index, although read in the inverse order, which thwarts attacks in which previous rows are discarded for saving memory and then recomputed right before they are used. In addition, thanks to the rot operation, each row receives slightly different outputs from the sponge, which reduces an attacker's ability to get useful results from XORing pairs of rows together. Notice that this rotation can be performed basically for free in software if $\omega$ is set to a multiple of $W$ as recommended: in this case, this operation corresponds to rearranging words rather than actually executing shifts or rotations. The left side of Figure 3 illustrates how the sponge's inputs and output are handled by Lyra2 during the Setup phase.

The initialization of $M[0]-M[2]$ in lines 8 to 10 , in contrast, is slightly different because none of them has enough predecessors to be treated exactly like the rows initialized during the Filling Loop. Specifically, instead of taking three

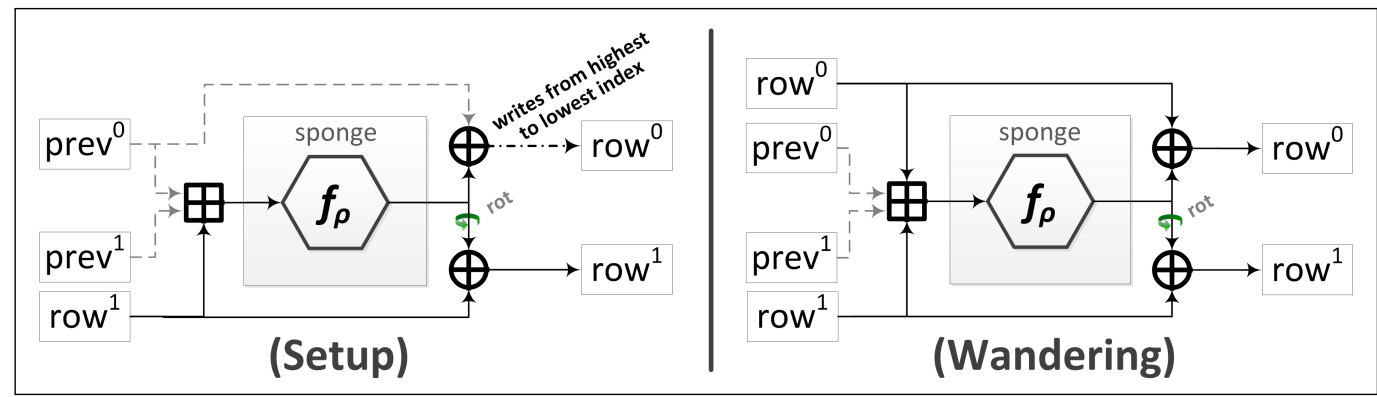

Figure 3: Handling the sponge's inputs and outputs during the Setup (left) and Wandering (right) phases in Lyra2. 
rows in the duplexing operation, $M[0]$ takes none while $M[1]$ and (for simplicity) $M[2]$ takes only their immediate predecessor.

The Setup phase ends when all $R$ rows of the memory matrix are initialized, which also means that any row ever indexed by row ${ }^{1}$ has also been updated since its initialization. These row $^{1}$ indices are deterministically picked from a window of size wnd, which starts with a single row and doubles in size whenever all of its rows are visited (i.e., whenever row $^{1}$ reaches the value 0 ). The exact values assumed by row ${ }^{1}$ depend on wnd, following a logic whose aim is to ensure that, if two rows are visited sequentially in one window, during the subsequent window they are visited (1) in points far away from each other and (2) approximately in the reverse order of their previous visitation. This hinders the recomputation of several values of $M\left[r_{0} w^{1}\right]$ from scratch in the sequence they are required, thwarting attacks that trade memory and processing costs, which are discussed in detail in Section 5.1. To accomplish this goal in a manner that is simple to implement, the following strategy was adopted (see Table 1):

- When $w n d$ is a square number: the window can be seen as a $\sqrt{w n d} \times \sqrt{w n d}$ matrix. Then, row ${ }^{1}$ is taken from the indices in that matrix's cyclic diagonals, starting with the main diagonal and moving right until the diagonal from the upper right corner is reached. This is accomplished by using a step variable $s t p=\sqrt{w n d}+1$, computed in line 21 of Algorithm 5, using the auxiliary sqrt $=\sqrt{w n d}$ variable to facilitate this computation.

- Otherwise: the window is represented as a $2 \sqrt{w n d / 2} \times \sqrt{w n d / 2}$ matrix. The values of row $^{1}$ start with 0 and then corresponding to the matrix's cyclic anti-diagonals, starting with the main anti-diagonal and cyclically moving left one column at a time. In this case, the step variable is computed as $s t p=2 \sqrt{w n d / 2}-1$ in the same line 21 of Algorithm 5, once again using the auxiliary $s q r t=2 \sqrt{w n d / 2}$ variable. 


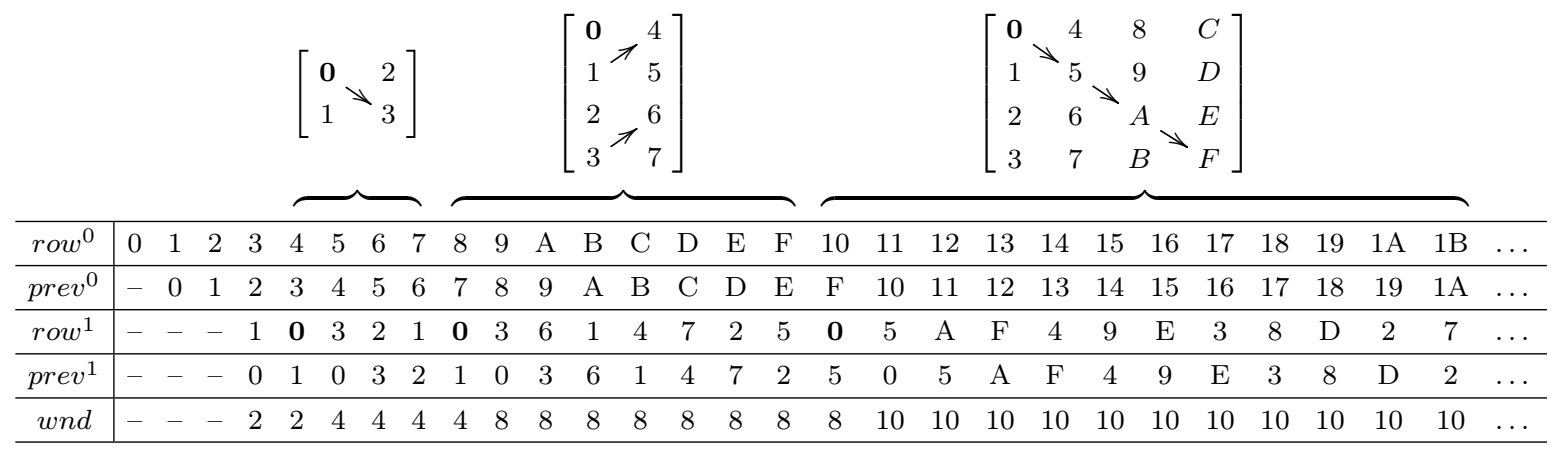

Table 1: Indices of the rows that feed the sponge when computing $M[$ row $]$ during Setup (hexadecimal notation).

Table 1 shows some examples of the values of row $^{1}$ in each iteration of the Filling Loop (lines 11-24), as well as the corresponding window size. We note that, since the window size is always a power of 2 , the modular operation in line 18 can be implemented with a simple bitwise AND with wnd - 1, potentially leading to better performance.

\subsubsection{The Wandering phase}

The most time-consuming of all phases, the Wandering Phase (lines 27 to 37), takes place after the Setup phase is finished, without resetting the sponge's internal state. Similarly to the Setup, the core of the Wandering phase consists in the reduced duplexing of rows that are added together (line 32) for computing a random-like output rand (line 32 ), which is then XORed with rows taken as input. One distinct aspect of the Wandering phase, however, refers to the way it handles the sponge's inputs and outputs, which is illustrated on the right side of Figure 3. Namely, besides taking four rows rather than three as input for the sponge, these rows are not all deterministically picked anymore, but all involve some kind of pseudorandom, password-dependent variable in their picking and visitation: 
- $\operatorname{row}^{d}(d=0,1)$ : indices computed in line 28 from the first and second words of the sponge's outer state, i.e., from $\operatorname{rand}[0]$ and $\operatorname{rand}[1]$ for $d=0$ and $d=1$, respectively. This particular computation ensures that each row $^{d}$ index corresponds to a pseudorandom value $\in[0, R-1]$ that is only learned after all columns of the previously visited row are duplexed. Given the wide range of possibilities, those rows are unlike to be in cache; however, since they are visited sequentially, their columns can be prefetched by the processor to speed-up their processing.

- $\operatorname{prev}^{d}(d=0,1)$ : set in line 36 to the indices of the most recently modified rows. Just like in the Setup phase, these rows are likely to still be in cache. Taking advantage of this fact, the visitation of its columns are not sequential but actually controlled by the pseudorandom, password-dependent variables $\left(\operatorname{col}^{0}, \operatorname{col}^{1}\right) \in[0, C-1]$. More precisely, each index $\operatorname{col}^{d}(d=0,1)$ is computed from the sponge's outer state; for example, for $\omega=W$, it is taken from $\operatorname{rand}[d+2]$ ) right before each duplexing operation (line 31). As a result, the corresponding column indices cannot be determined prior to each duplexing, forcing all the columns to remain in memory for the whole duplexing operation for better performance and thwarting the construction of simple pipelines for their visitation.

The treatment given to the sponge's outputs is then quite similar to that in the Setup phase: the outputs provided by the sponge are sequentially XORed with $M\left[\right.$ row $\left.^{0}\right]$ (line 33) and, after being rotated, with $M\left[\right.$ row $\left.^{1}\right]$ (line 34). However, in the Wandering phase the sponge's output is XORed with $M\left[r_{0} w^{0}\right]$ from the lowest to the highest index, just like $M\left[\right.$ row $\left.^{1}\right]$. This design decision was adopted because it allows faster processing, since the columns read are also those overwritten; at the same time, the subsequent reading of those columns in a pseudorandom order already thwarts the attack strategy discussed in Section 5.1.2.4, so there is no need 
to revert the reading/writing order in this part of the algorithm.

\subsubsection{The Wrap-up phase}

Finally, after $(R \cdot T)$ duplexing operations are performed during the Wandering phase, the algorithm enters the Wrap-up Phase. This phase consists of a full-round absorbing operation (line 39) of a single cell of the memory matrix, $M\left[r^{0} w^{0}\right][0]$. The goal of this final call to absorb is mainly to ensure that the squeezing of the key bitstring will only start after the application of one full-round $f$ to the sponge's state - notice that, as shown in Figure 1, the squeezing phase starts with $b$ bits being output rather than passing by $f$ and, since the full-round absorb in line 4, the state was only updated by several calls to the reduced-round $f$. This absorb operation is then followed by a full-round squeezing operation (line 40) for generating $k$ bits, once again without resetting sponge's internal state to zeros. As a result, this last stage employs only the regular operations of the underlying sponge, building on its security to ensure that the whole process is both non-invertible and the outputs are unpredictable. After all, violating such basic properties of Lyra2 is equivalent to violate the same basic properties of the underlying full-round sponge.

\subsection{Strictly sequential design}

Like with PBKDF2 and other existing PHS, Lyra2's design is strictly sequential, as the sponge's internal state is iteratively updated during its operation. Specifically, and without loss of generality, assume that the sponge's state before duplexing a given input $c_{i}$ is $s_{i}$; then, after $c_{i}$ is processed, the updated state becomes $s_{i+1}=f_{\rho}\left(s_{i} \oplus c_{i}\right)$ and the sponge outputs $\operatorname{rand}_{i}$, the first $b$ bits of $s_{i+1}$. Now, suppose the attacker wants to parallelize the duplexing of multiple columns in lines 13-17 (Setup phase) or in lines 29-35 (Wandering phase), obtaining 
$\left\{\operatorname{rand}_{0}, \operatorname{rand}_{1}, \operatorname{rand}_{2}\right\}$ faster than sequentially computing $\operatorname{rand}_{0}=f_{\rho}\left(s_{0} \oplus c_{0}\right)$, $\operatorname{rand}_{1}=f_{\rho}\left(s_{1} \oplus c_{1}\right)$, and then $\operatorname{rand}_{2}=f_{\rho}\left(s_{2} \oplus c_{2}\right)$.

If the sponge's transformation $f$ was affine, the above task would be quite easy. For example, if $f_{\rho}$ was the identity function, the attacker could use two processing cores to compute $\operatorname{rand}_{0}=s_{0} \oplus c_{0}, x=c_{1} \oplus c_{2}$ in parallel and then, in a second step, make $\operatorname{rand}_{1}=\operatorname{rand}_{0} \oplus c_{1}, \operatorname{rand}_{2}=\operatorname{rand}_{0} \oplus x$ also in parallel. With dedicated hardware and adequate wiring, this could be done even faster, in a single step. However, for a highly non-linear transformation $f_{\rho}$, it should be hard to decompose two iterative duplexing operations $f_{\rho}\left(f_{\rho}\left(s_{0} \oplus c_{0}\right) \oplus c_{1}\right)$ into an efficient parallelizable form, let alone several applications of $f_{\rho}$.

It is interesting to notice that, if $f_{\rho}$ has some obvious cyclic behavior, always resetting the sponge to a known state $s$ after $v$ cells are visited, then the attacker could easily parallelize the visitation of $c_{i}$ and $c_{i+v}$. Nonetheless, any reasonably secure $f_{\rho}$ is expected to prevent such cyclic behavior by design, since otherwise this property could be easily explored for finding internal collisions against the full $f$ itself.

In summary, even though an attacker may be able to parallelize internal parts of $f_{\rho}$, the stateful nature of Lyra2 creates several "serial bottlenecks" that prevent duplexing operations from being executed in parallel.

Assuming that the above-mentioned structural attacks are unfeasible, parallelization can still be achieved in a "brute-force" manner. Namely, the attacker could create two different sponge instances, $I_{0}$ and $I_{1}$, and try to initialize their internal states to $s_{0}$ and $s_{1}$, respectively. If $s_{0}$ is known, all the attacker needs to do is compute $s_{1}$ faster than actually duplexing $c_{0}$ with $I_{0}$. For example, the attacker could rely on a large table mapping states and input blocks to the resulting states, and then use the table entry $\left(s_{0}, c_{0}\right) \mapsto s_{1}$. For any reasonable cryptographic sponge, however, the state and block sizes are expected to be quite 
large (e.g., 512 or 1,024 bits), meaning that the amount of memory required for building a complete map makes this approach unpractical.

Alternatively, the attacker could simply initialize several $I_{1}$ instances with guessed values of $s_{1}$, and use them to duplex $c_{1}$ in parallel. Then, when $I_{0}$ finishes running and the correct value of $s_{1}$ is inevitably determined, the attacker could compare it to the guessed values, keeping only the result obtained with the correct instantiation. At first sight, it might seem that a reduced-round $f$ facilitates this task, since the consecutive states $s_{0}$ and $s_{1}$ may share some bits or relationships between bits, thus reducing the number of possibilities that need to be included among the guessed states. Even if that is the case, however, any transformation $f$ is expected to have a complex relation between the input and output of every single round and, to speed-up the duplexing operation, the attacker needs to explore such relationship faster than actually processing $\rho$ rounds of $f$. Otherwise, the process of determining the target guessing space will actually be slower than simply processing cells sequentially. Furthermore, to guess the state that will be reached after $v$ cells are visited, the attacker would have to explore relationships between roughly $v \cdot \rho$ rounds of $f$ faster than merely running $v \cdot \rho$ rounds of $f_{\rho}$. Hence, even in the (unlikely) case that guessing two consecutive states can be made faster than running $\rho$ of $f$, this strategy scales poorly since any existing relationship between bits should be diluted as $v \cdot \rho$ approaches $\rho_{\max }$.

An analogous reasoning applies to the Filling / Visitation Loop. The only difference is that, to parallelize the duplexing of inputs from its consecutive iterations, $c_{i}$ and $c_{i+1}$, the attacker needs to determine the sponge's internal state $s_{i+1}$ that will result from duplexing $c_{i}$ without actually performing the $C \cdot \rho$ rounds of $f$ involved in this operation. Therefore, even if highly parallelizable hardware is available to attackers, it is unlikely that they will be able to take full advantage of this potential for speeding up the operation of any given instance of Lyra2. 


\subsection{Configuring memory usage and processing time}

The total amount of memory occupied by Lyra2's memory matrix is $b \cdot R \cdot C$ bits, where $b$ corresponds to the underlying sponge function's bitrate. With this choice of $b$, there is no need to pad the incoming blocks as they are processed by the duplex construction, which leads to a simpler and potentially faster implementation. The $R$ and $C$ parameters, on the other hand, can be defined by the user, thus allowing the configuration of the amount of memory required during the algorithm's execution.

Ignoring ancillary operations, the processing cost of Lyra2 is basically determined by the number of calls to the sponge's underlying $f$ function. Its approximate total cost is, thus: $\lceil(|p w d|+\mid$ salt $|+|$ params $\mid) / b\rceil$ calls in Bootstrapping phase, plus $R \cdot C \cdot \rho / \rho_{\max }$ in the Setup phase, plus $T \cdot R \cdot C \cdot \rho / \rho_{\max }$ in the Wandering phase, plus $\lceil k / b\rceil$ in the Wrap-up phase, leading roughly to $(T+1) \cdot R \cdot C \cdot \rho / \rho_{\max }$ calls to $f$ for small lengths of $p w d$, salt and $k$. Therefore, while the amount of memory used by the algorithm imposes a lower bound on its total running time, the latter can be increased without affecting the former by choosing a suitable $T$ parameter. This allows users to explore the most abundant resource in a (legitimate) platform with unbalanced availability of memory and processing power. This design also allows Lyra2 to use more memory than scrypt for a similar processing time: while scrypt employs a full-round hash for processing each of its elements, Lyra2 employs a reduced-round, faster operation for the same task.

\subsection{On the underlying sponge}

Even though Lyra2 is compatible with any hash functions from the sponge family, the newly approved SHA-3, Keccak (BERTONI et al., 2011b), does not 
seem to be the best alternative for this purpose. This happens because Keccak excels in hardware rather than in software performance (GAJ et al., 2012). Hence, for the specific application of password hashing, it gives more advantage to attackers using custom hardware than to legitimate users running a software implementation.

Our recommendation, thus, is toward using a secure software-oriented algorithm as the sponge's $f$ transformation. One example is Blake2b (AUMASSON et al., 2013), a slightly tweaked version of Blake (AUMASSON et al., 2010b). Blake itself displays a security level similar to that of Keccak (CHANG et al., 2012), and its compression function has been shown to be a good permutation (AUMASSON et al., 2010a; MING; QIANG; ZENG, 2010) and to have a strong diffusion capability (AUMASSON et al., 2010b) even with a reduced number of rounds (JI; LIANGYU, 2009; SU et al., 2010), while Blake2b is believed to retain most of these security properties (GUO et al., 2014).

The main (albeit minor) issue with Blake2b's permutation is that, to avoid fixed points, its internal state must be initialized with a 512-bit initialization vector (IV) rather than with a string of zeros as prescribed by the sponge construction. The reason is that Blake2b does not use the constants originally employed in Blake2 inside its G function (AUMASSON et al., 2013), relying on the IV for avoiding possible fixed points. Indeed, if the internal state is filled with zeros as usually done in cryptographic sponges, any block filled with zeros absorbed by the sponge will not change this state value. Therefore, the same IV should also be used for initializing the sponge's state in Lyra2. In addition, to prevent the IV from being overwritten by user-defined data, the sponge's capacity $c$ employed when absorbing the user's input (line 4 of Algorithm 5) should have at least 512 bits, leaving up to 512 bits for the bitrate $b$. After this first absorb, though, the bitrate may be raised for increasing the overall throughput of Lyra2 if so desired. 


\subsubsection{A dedicated, multiplication-hardened sponge: BlaMka.}

Besides plain Blake2b, another potentially interesting alternative is to employ a permutation that involves integer multiplications among its operations, following the "multiplication-hardening" concept (COX, 2014; PESLYAK, 2015) briefly mentioned in Section 3.2.5 when discussing the yescrypt PHC candidate. More precisely, this approach is of interest whenever a legitimate user prefers to rely on a function that provides further protection against dedicated hardware platforms, while maintaining a high efficiency on platforms such as CPUs.

For this purpose the Blake2b structure may itself be adapted to integrate multiplications, which is done in the hereby proposed BlaMka algorithm. More precisely, Blake2b's $G$ function (see the left side of Figure 4) relies on sequential additions, rotations and XORs (ARX) for attaining bit diffusion and creating a mutual dependence between those bits (AUMASSON et al., 2010a; MING; QIANG; ZENG, 2010). If, however, the additions employed are replaced by another permutation that includes multiplications and still provides at least the same capacity of diffusion, its security should not be negatively affected.

$$
\begin{array}{rlrl}
a & \leftarrow a+b & a & \leftarrow a+b+2 \cdot \operatorname{lsw}(a) \cdot \operatorname{lsw}(b) \\
d & \leftarrow(d \oplus a) \ggg 32 & d & \leftarrow(d \oplus a) \ggg 32 \\
c & \leftarrow c+d & c & \leftarrow c+d+2 \cdot \operatorname{lsw}(c) \cdot \operatorname{lsw}(d) \\
b & \leftarrow(b \oplus c) \ggg 24 & b & \leftarrow(b \oplus c) \ggg 24 \\
a & \leftarrow a+b & a & \leftarrow a+b+2 \cdot \operatorname{lsw}(a) \cdot \operatorname{lsw}(b) \\
d & \leftarrow(d \oplus a) \ggg 16 & d & \leftarrow(d \oplus a) \ggg 16 \\
c & \leftarrow c+d & c & \leftarrow c+d+2 \cdot \operatorname{lsw}(c) \cdot \operatorname{lsw}(d) \\
b & \leftarrow(b \oplus c) \ggg 63 & b & \leftarrow(b \oplus c) \ggg 63 \\
\begin{array}{cl}
\text { (a) Blake2b's function, } \\
\text { based on the addition }
\end{array} & & \text { (b) BlaMka's } G_{\mathrm{tls}} \text { function, based on a } \\
& \text { operation. } & & \text { truncated latin square (tls). }
\end{array}
$$

Figure 4: BlaMka's multiplication-hardened (right) and Blake2b's original (left) permutations. 
One suggestion, originally made by Samuel Neves (one of the authors of Blake2) (NEVES, 2014), was to replace the additions of integers $x$ and $y$ by something like the latin square function (WALLIS; GEORGE, 2011) 1s $(x, y)=$ $x+y+2 \cdot x \cdot y$. This would lead to a structure quite similar to what is done in the NORX authenticated encryption scheme (AUMASSON; JOVANOVIC; NEVES, 2014), but in the additive field. To make it friendlier for implementation using the instruction set of modern processors, however, one can use a slightly modified construction that employs the least significant bits of $x$ and $y$, which can be seen as a truncated version of the latin square. Some alternatives (one of which is described in Appendix $\mathrm{C}$ of this document) have been evaluated, but the end result is $\operatorname{tls}(x, y)=x+y+2 \cdot \operatorname{lsw}(x) \cdot \operatorname{lsw}(y)$, as shown on the right side of Figure 4 for the $G_{\mathrm{t} l \mathrm{~s}}$ function. This tls operation can be efficiently implemented using fast SIMD instructions (e.g., _MM_MUL_EPU, _MM_SLLI_EPI, _MM_ADD_EPI),

and keeps an homogeneous distribution for the $\mathbb{F}_{2}^{2 n} \mapsto \mathbb{F}_{2}^{n}$ mapping (i.e., it still is a permutation).

The resulting structure, whose security and performance are further analyzed later in Sections 5.5 and 6.2, is indeed quite promising for usage in password hashing schemes Specifically, it provides better performance on hardware than on software platforms than Blake2 itself, justifying its early adoption as the default underlying sponge of Argon2's official implementation (PHC, 2015a) and also of Lyra2.

\subsection{Practical considerations}

Lyra2 displays a quite simple structure, building as much as possible on the intrinsic properties of sponge functions operating on a fully stateful mode. Indeed, the whole algorithm is basically composed of loop controlling and variable initialization statements, while the data processing itself is done by the underlying 
hash function $H$. Therefore, we expect the algorithm to be easily implementable in software, especially if a sponge function is already available.

The adoption of sponges as underlying primitive also gives Lyra2 great flexibility. For example, since the user's input (line 4 of Algorithm 3) is processed by an absorb operation, the length and contents of such input can be easily chosen by the user, as previously discussed. Likewise, the algorithm's output is computed using the sponge's squeezing operation, allowing any number of bits to be securely generated without the need of another primitive (e.g., PBKDF2, as done in scrypt).

Another feature of Lyra2 is that its memory matrix was designed to allow legitimate users to take advantage of memory hierarchy features, such as caching and prefetching. As observed in (PERCIVAL, 2009), such mechanisms usually make access to consecutive memory locations in real-world machines much faster than accesses to random positions, even for memory chips classified as "random access". As a result, a memory matrix having a small $R$ is likely to be visited faster than a matrix having a small $C$, even for identical values of $R \cdot C$. Therefore, by choosing adequate $R$ and $C$ values, Lyra2 can be optimized for running faster in the target (legitimate) platform while still imposing penalties to attackers under different memory-accessing conditions. For example, by matching $b \cdot C$ to approximately the size of the target platform's cache lines, memory latency can be significantly reduced, allowing $T$ to be raised without impacting the algorithm's performance in that specific platform.

Besides performance, making $C \geqslant \rho_{\max }$ is also recommended for security reasons: as discussed in Section 4.2, this parametrization ensures that the sponge's internal state is scrambled with (at least) the full strength of the underlying hash function after the execution of the Setup or Wandering phase's Columns Loops. The task of guessing the sponge's state after the conclusion of any iteration of 
a Columns Loop without actually executing it becomes, thus, much harder. After all, assuming the underlying sponge can be modeled as a random oracle, its internal state should be indistinguishable from a random bitstring.

One final practical concern taken into account in the design of Lyra2 refers to how long the original password provided by the user needs to remain in memory. Specifically, the memory position storing pwd can be overwritten right after the first absorb operation (line 4 of Algorithm 5). This avoids situations in which a careless implementation ends up leaving $p w d$ in the device's volatile memory or, worse, leading to its storage in non-volatile memory due to memory swaps performed during the algorithm's memory-expensive phases. Hence, it meets the general guideline of purging private information from memory as soon as it is not needed anymore, preventing that information's recovery in case of unauthorized access to the device (HALDERMAN et al., 2009; YUILL; DENNING; FEER, 2006). 


\section{SECURITY ANALYSIS}

Lyra2's design is such that (1) the derived key is both non-invertible and collision resistant, which is due to the initial and final full hashing operations, combined with reduced-round hashing operations in the middle of the algorithm; (2) attackers are unable to parallelize Algorithm 5 using multiple instances of the cryptographic sponge $H$, so they cannot significantly speed up the process of testing a password by means of multiple processing cores; (3) once initialized, the memory matrix is expected to remain available during most of the password hashing process, meaning that the optimal operation of Lyra2 requires enough (fast) memory to hold its contents.

For better performance, a legitimate user is likely to store the whole memory matrix in volatile memory, facilitating its access in each of the several iterations of the algorithm. An attacker running multiple instances of Lyra2, on the other hand, may decide not to do the same, but to keep a smaller part of the matrix in fast memory aiming to reduce the memory costs per password guess. Even though this alternative approach inevitably lowers the throughput of each individual instance of Lyra2, the goal with this strategy is to allow more guesses to be independently tested in parallel, thus potentially raising the overall throughput of the process.

There are basically two methods for accomplishing this. The first is what we call a Low-Memory attack, which consists of trading memory for processing time, 
i.e., discarding (parts of) the matrix and recomputing the discarded information from scratch, when (and only when) it becomes necessary. The second it to use low-cost (and, thus, slower) storage devices, such as magnetic hard disks, which we call a Slow-Memory attack.

In what follows, we discuss both attack venues and evaluate their relative costs, as well as the drawbacks of such alternative approaches. Our goal with this discussion is to demonstrate how Lyra2's design discourages attackers from making such memory-processing trade-offs while testing many passwords in parallel. Consequently, the algorithm limits the attackers' ability to take advantage of highly parallel platforms, such as GPUs and FPGAs, for password cracking.

In addition to the above attacks, the security analysis hereby presented also discusses the so-called Cache-Timing attacks (FORLER; LUCKS; WENZEL, 2013), which employ a spy process collocated to the PHS and, by observing the latter's execution, could be able to recover the user's password without the need of engaging in an exhaustive search. It also evaluates Lyra2 in terms of GarbageCollector attacks (FORLER et al., 2014), an attack that explores vulnerabilities of the memory management during the derivation process. Finally, we present a preliminary analysis of the BlaMka sponge-based hash function proposed in Section 4.4.1.

\subsection{Low-Memory attacks}

Before we discuss low-memory attacks against Lyra2, it is instructive to consider how such attacks can be perpetrated against scrypt's ROMix structure (see Algorithm 3). The reason is that its sequential memory hard design is mainly intended to provide protection against this particular attack venue. 
As a direct consequence of scrypt's memory hard design, we can formulate Theorem 1:

Theorem 1. Whilst the memory and processing costs of scrypt are both $O(R)$ for a system parameter $R$, one can achieve a memory cost of $O(1)$ (i.e., a memoryfree attack) by raising the processing cost to $O\left(R^{2}\right)$.

Proof. The attacker runs the loop for initializing the memory array $M$ (lines 9 to 11 of Algorithm 3), which we call ROMix $x_{i n i}$. Instead of storing the values of $M[i]$, however, the attacker keeps only the value of the internal variable $X$. Then, whenever an element $M[j]$ of $M$ should be read (line 14 of Algorithm 3), the attacker simply runs $R O M i x_{i n i}$ for $j$ iterations, determining the value of $M[j]$ and updating $X$. Ignoring ancillary operations, the average cost of such attack is $R+(R \cdot R) / 2$ iterative applications of BlockMix and the storage of a single $b$-long variable $(X)$, where $R$ is scrypt's cost parameter.

In comparison, an attacker trying to use a similar low-memory attack against Lyra2 would run into additional challenges. First, during the Setup phase, it is not enough to keep only one row in memory for computing the next one, as each row requires three previously computed rows for its computation.

For example, after using $M[0]-M[2]$, those three rows are once again employed in the computation of $M[3]$, meaning that they should not be discarded or they will have to be recomputed. Even worse: since $M[0]$ is modified when initializing $M[4]$, the value to be employed when computing rows that depend on it (e.g., $M[8]$ ) cannot be obtained directly from the password. Instead, recomputing the updated value of $M[0]$ requires (a) running the Setup phase until the point it was last modified (e.g., for the value required by $M[8]$, this corresponds to when $M[4]$ was initialized) or (b) using some rows still available in memory, XORing them together to obtain the values of $\operatorname{rand}[\mathrm{col}]$ that modified $M[0]$ since its 
initialization.

Whichever the case, this creates a complex net of dependencies that grow in size as the algorithm's execution advances and more rows are modified, leading to several recursive calls. This effect is even more expressive in the Wandering phase, due to an extra complicating factor: each duplexing operation involves a random-like (password-dependent) row index that cannot be determined before the end of the previous duplexing. Therefore, the choice of which rows to keep in memory and which rows to discard is merely speculative, and cannot be easily optimized for all password guesses.

Providing a tight bound for the complexity of such low-memory attacks against Lyra2 is, thus, an involved task, especially considering its nondeterministic nature. Nevertheless, aiming to give some insight on how an attacker could (but is unlikely to want to) explore such time-memory trade-offs, in what follows we consider some slightly simplified attack scenarios. We emphasize, however, that these scenarios are not meant to be exhaustive, since the goal of analyzing them is only to show the approximate (sometimes asymptotic) impact of possible memory usage reductions over the algorithm's processing cost.

Formally proving the resistance of Lyra2 against time-memory trade-offs e.g., using the theory of Pebble Games (COOK, 1973; DWORK; NAOR; WEE, 2005) as done in (FORLER; LUCKS; WENZEL, 2013; DZIEMBOWSKI; KAZANA; WICHS, 2011) — would be even better, but doing so, possibly building on the discussion hereby presented, remains as a matter for future work.

\subsubsection{Preliminaries}

For conciseness, along the discussion we denote by $C L$ the Columns Loop of the Setup phase (lines 13-17 of Algorithm 5) and of the Wandering phase (lines $29-35)$. In this manner, ignoring the cost of XORing, reads/writes and other 
ancillary operations, $C L$ corresponds approximately to $C \cdot \rho / \rho_{\max }$ executions of $f$, a cost that is denoted simply as $\sigma$.

We denote by $s_{i, j}^{0}$ the state of the sponge right before $M[i][j]$ is initialized in the Setup phase. For $i \geqslant 3$, this corresponds to the state in line 13 of Algorithm 5. For conciseness, though, we often omit the " $j$ " subscript, using $s_{i}^{0}$ as a shorthand for $s_{i, 0}^{0}$ whenever the focus of the discussion are entire rows rather than their cells. We also employ a similar notation for the Wandering phase, denoting by $s_{i}^{\tau}$ the state of the sponge during iteration $R \cdot(\tau-1)+i$ of the Visitation Loop (with $1 \leqslant \tau \leqslant T$ ), before the corresponding rows are effectively processed (i.e., the state in line 27 of Algorithm 5). Analogously, the $i$-th row $(0 \leqslant i<R)$ output by the sponge during the Setup phase is denoted $r_{i}^{0}$, while $r_{i}^{\tau}$ denotes the output given by the Visitation Loop's iteration $R \cdot(\tau-1)+i$. In this manner, the $\tau$ symbol is employed to indicate how many times the Wandering phase performs a number of duplexing operations equivalent to that in the Setup phase.

Aiming to keep track of modifications made on rows of the memory matrix, we recursively use the subscript notation $M\left[X_{Y-Z-\ldots}\right]$ to denote a row $X$ modified when it received the same values of rand as row $Y$, then again when the row receiving the sponge's output was $Z$, and so on. For example, $M\left[1_{3}\right]$ corresponds to row $M[1]$ after its cells are XORed with $\operatorname{rot}($ rand $)$ in the very first iteration of the Setup phase's Filling Loop. Finally, for conciseness, we write $V_{1}^{\tau}$ and $V_{2}^{\tau}$ to denote, respectively, the first and second half of: the Setup phase, for $\tau=0$; or the Visitation Loop during iteration $R \cdot(\tau-1)+i$ of the Wandering phase's Visitation Loop, for $\tau \geqslant 1$.

\subsubsection{The Setup phase}

We start our discussion analyzing only the Setup phase. Aiming to give a more concrete view of its execution, along the discussion we use as an example 


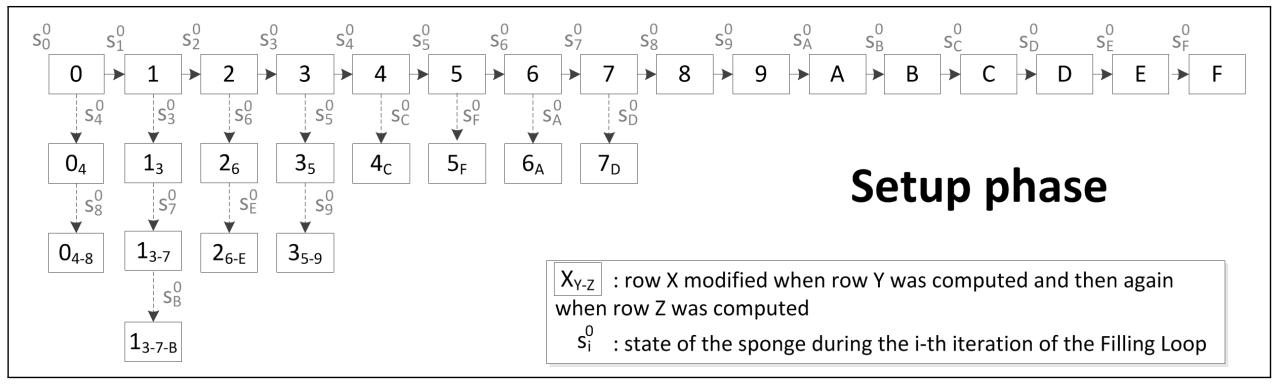

Figure 5: The Setup phase.

the scenario with 16 rows depicted in Figure 5, which shows the corresponding visitation order of such rows and also their modifications due to these visitations.

\subsubsection{Storing only what is needed: $1 / 2$ memory usage}

Suppose that the attacker does not want to store all rows of the memory matrix during the algorithm's execution. One interesting approach for doing so is to keep in buffer only what will be required in future iterations of the Filling Loop, discarding rows that will not be used anymore. Since the Setup phase is purely deterministic, doing so is quite easy and, as long as the proper rows are kept, it incurs no processing penalty. This approach is illustrated in Figure 6 .

As shown in this figure, this simple strategy allows the execution of the Setup phase with a memory usage of $R / 2+1$ rows, approximately half of the amount

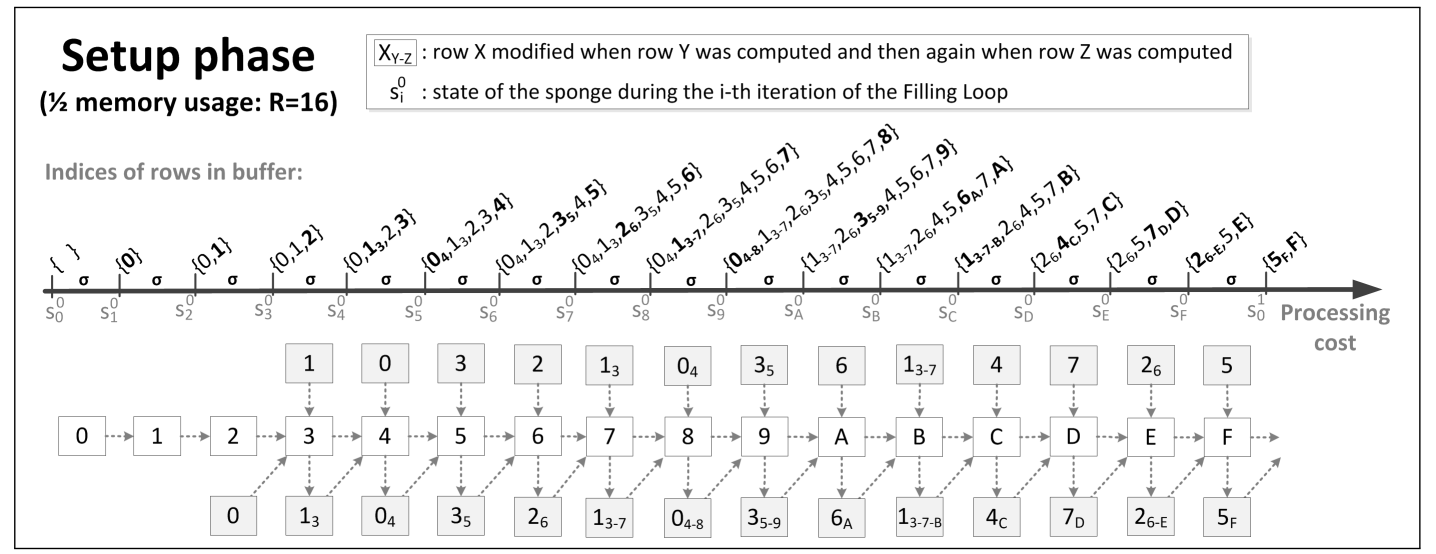

Figure 6: Attacking the Setup phase: storing 1/2 of all rows. The most recently modified rows in each iteration are marked in bold. 
usually required. This observation comes from the fact that each half of the Setup phase requires all rows from the previous half and two extra rows (those more recently initialized/updated) to proceed. More precisely, $R / 2+1$ corresponds to the peak memory utilization reached around the middle of the Setup phase, since (1) until then, part of the memory matrix has not been initialized yet and (2) rows initialized near the end of the Setup phase are only required for computing the next row and, thus, can be overwritten right after their cells are used. Even with this reduced memory usage, the processing cost of this phase remains at $R \cdot \sigma$, just as if all rows were kept in memory.

This attack can, thus, be summarized by the following lemma:

Lemma 1. Consider that Lyra2 operates with parameters $T, R$ and $C$. Whilst the regular algorithm's memory and processing costs of its Setup phase are, respectively, $R \cdot C \cdot b$ bits and $R \cdot \sigma$, it is possible to run this phase with a maximum memory cost of approximately $(R / 2) \cdot C \cdot b$ bits while keeping its total processing cost to $R \cdot \sigma$.

Proof. The costs involved in the regular operation of Lyra2 are discussed in Section 4.3, while the mentioned memory-processing trade-off can be achieved with the attack described in this section.

\subsubsection{Storing less than what is needed: $1 / 4$ memory usage}

If the attacker considers that storing half of the memory matrix is too much, he/she may decide to discard additional rows, recomputing them from scratch only when they are needed. In that case, a reasonable approach is to discard rows that (1) will take longer to be used, either directly or for the recomputation of other rows, or (2) that can be easily computed from rows already available, so the impact of discarding them is low. The reasoning behind this strategy is that it allows the Setup phase to proceed smoothly for as long as possible. Therefore, 


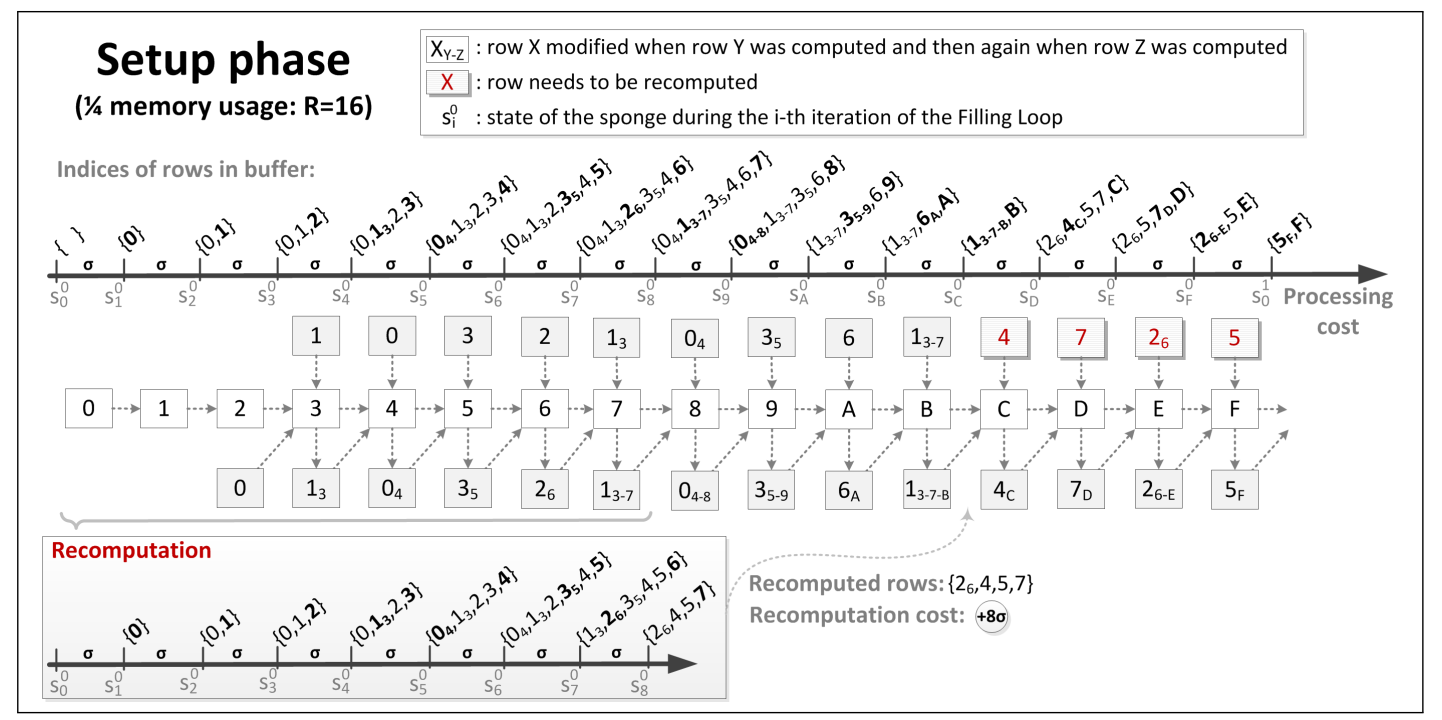

Figure 7: Attacking the Setup phase: storing 1/4 of all rows. The most recently modified rows in each iteration are marked in bold.

as rows that are not too useful for the time being (or even not required at all anymore) are discarded from the buffer, the space saved in this manner can be diverted to the recomputation process, accelerating it.

The suggested approach is illustrated in Figure 7. As shown in this figure, at any moment we keep in memory only $R / 4=4$ rows of the memory matrix besides the two most recently modified/updated, approximately half of what is used in the attack described in Section 5.1.2.1. This allows roughly $3 / 4$ of the Setup phase to run without any recomputation, but after that $M[4]$ is required to compute row $M[C]$. One simple way of doing so is to keep in memory the two most recently modified rows, $M\left[1_{3-7-B}\right]$ and $M[B]$, and then run the first half of the Setup phase once again with $R / 4+2$ rows. This strategy should allow the recomputation not only of $M[4]$, but of all the $R / 4$ rows previously discarded but still needed for the last 1/4 of the Setup phase (in our example, $\left\{M[4], M[7], M\left[2_{6}\right], M[5]\right\}$, as shown at the bottom of Figure 7). The resulting processing overhead would, thus, be approximately $(R / 2) \sigma$, leading to a total cost of $(3 R / 2) \sigma$ for the whole Setup.

Obviously, there may be other ways of recomputing the required rows. For 
example, there is no need to discard $M[7]$ after $M[8]$ is computed, since keeping it in the buffer after that point would still respect the $R / 4+2$ memory cost. Then, the recomputation procedure could stop after the recomputation of $M\left[2_{6}\right]$, reducing its cost in $\sigma$. Alternatively, $M[4]$ could have been kept in memory after the computation of $M[7]$, allowing the recomputations to be postponed by one iteration. However, then $M[7]$ could not be maintained as mentioned above and there would be not reduction in the attack's total cost. All in all, these and other tricks are not expected to reduce the total recomputation overhead significantly below $(R / 2) \sigma$. This happens because the last $1 / 4$ of the Setup phase is designed in such a manner that the $r_{0} w^{1}$ index covers the entire first half of the memory matrix, including values near 0 and $R / 2$. As a result, the recomputation of all values of $M\left[r_{0} w^{1}\right]$ input to the sponge near the end of the Setup phase is likely to require most (if not all) of its first half to be executed.

These observations can be summarized in the following conjecture.

Conjecture 1. Consider that Lyra2 operates with parameters $T, R$ and $C$. Whilst the regular memory and processing costs of its Setup phase's are, respectively, $\operatorname{MemSetup}(R)=R \cdot C \cdot b$ bits and $\operatorname{CostSetup}(R)=R \cdot \sigma$, its execution with a memory cost of approximately $\operatorname{MemSetup}(R) / 4$ should raise its processing cost to approximately 3 Cost $\operatorname{Setup}(R) / 2$.

\subsubsection{Storing less than what is needed: $1 / 8$ memory usage}

We can build on the previous analysis to estimate the performance penalty incurred when reducing the algorithm's memory usage by another half. Namely, imagine that Figure 7 represents the first half of the Setup phase (denoted $V_{1}^{0}$ ) for $R=32$, in an attack involving a memory usage of $R / 8=4$. In this case, recomputations are needed after approximately $3 / 8$ of the Setup phase is executed. However, these are not the only recomputations that will occur, as the 
entire second half of the memory matrix (i.e., $R / 2$ rows) still needs to be initialized during the second half of the Setup phase (denoted $V_{2}^{0}$ ). Therefore, the $R / 2$ rows initialized/modified during $V_{1}^{0}$ will be once again required. Now suppose that the $R / 8$ memory budget is employed in the recomputation of the required rows from scratch, running $V_{1}^{0}$ again whenever a group of previously discarded rows is needed. Since a total of $R / 2$ rows need recomputation, the goal is to recover each of the $(R / 2) /(R / 8)=4$ groups of $R / 8$ rows in the sequence they are required during $V_{2}^{0}$, similarly to what was done a single time when the memory committed to the attack was $R / 4$ rows (section 5.1.2.2). In our example, the four groups of rows required are (see Table 1): $g_{1}=\left\{M\left[0_{4-8}\right], M[9], M\left[2_{6-E}\right], M[B]\right\}$, $g_{2}=\left\{M\left[4_{C}\right], M[D], M\left[6_{A}\right], M[F]\right\}, g_{3}=\left\{M[8], M\left[1_{3-7-B}\right], M[A], M\left[3_{5-9}\right]\right\}$, and $g_{4}=\left\{M[C], M\left[5_{F}\right], M[E], M\left[7_{D}\right]\right\}$, in this sequence.

To analyze the cost of this strategy, assume initially that the memory budget of $R / 8$ is enough to recover each of these groups by means of a single (partial or full) execution of $V_{1}^{0}$. First, notice that the computation of each group from scratch involves a cost of at least $(R / 4) \sigma$, since the rows required by $V_{2}^{0}$ have all been initialized or modified after the execution of $50 \%$ of $V_{1}^{0}$. Therefore, the lowest cost for recovering any group is $(3 R / 8) \sigma$, which happens when that group involves only rows initialized/modified before $M[R / 4+R / 8]$ (this is the case of $g_{3}$ in our example). A full execution of $V_{1}^{0}$, on the other hand, can be obtained from Conjecture 1: the buffer size is $\operatorname{MemSetup}(R / 2) / 4=R / 8$ rows, which means that the processing cost is now $3 \operatorname{CostSetup}(R / 2) / 2=(3 R / 4) \sigma$ (in our example, full executions are required for $g_{2}$ and $g_{4}$, due to rows $M[F]$ and $\left.M\left[5_{F}\right]\right)$. From these observations, we can estimate the four re-executions of $V_{1}^{0}$ to cost between $4(3 R / 8) \sigma$ and $4(3 R / 4) \sigma$, leading to an arithmetic mean of $(9 R / 4) \sigma$. Considering that a full execution of $V_{1}^{0}$ occurs once before $V_{2}^{0}$ is reached, and that $V_{2}^{0}$ itself involves a cost of $(R / 2) \sigma$ even without taking the above overhead into 
account, the base cost of the Setup phase is $(3 R / 4+R / 2) \sigma$. With the overhead of $(9 R / 4) \sigma$ incurred by the re-executions of $V_{1}^{0}$, the cost of the whole Setup phase then becomes $(7 R / 2) \sigma$.

We emphasize, however, that this should be seen a coarse estimate, since it considers four (roughly complementary) factors described in what follows.

1. The one-to-one proportion between a full and a partial execution of $V_{1}^{0}$ when initializing rows of $V_{2}^{0}$ is not tight. Hence, estimating costs with the arithmetic mean as done above may not be strictly correct. For example, going back to our scenario with $R=32$ and a $R / 8$ memory usage, the only group whose rows are all initialized/modified before $M[R / 2-R / 8]=M[C]$ is $g_{3}$. Therefore, this is the only group that can be computed by running the part of $V_{1}^{0}$ that does not require internal recomputations. Consequently, the average processing cost of recomputing those groups during $V_{2}^{0}$ should be higher.

2. As discussed in section 5.1.2.2, the attacker does not necessarily need to always compute everything from scratch. After all, the committed memory budget can be used to bufferize a few rows from $V_{1}^{0}$, avoiding the need of recomputing them. Going back to our example with $R=32$ and $R / 8$ rows, if $M\left[2_{6-E}\right]$ remains available in memory when $V_{2}^{0}$ starts, $g_{1}$ can be recovered by running $V_{1}^{0}$ once, until $M[B]$ is computed, which involves no internal recomputations. This might reduce the average processing cost of recomputations, possibly compensating the extra cost incurred by factor 1 .

3. The assumption that each of the four executions of $V_{1}^{0}$ can recover an entire group with the costs hereby estimated is not always realistic. The reason is that the costs of $V_{1}^{0}$ as described in section 5.1.2.2 are attained when what is kept in memory is only the set of rows strictly required during $V_{1}^{0}$. 


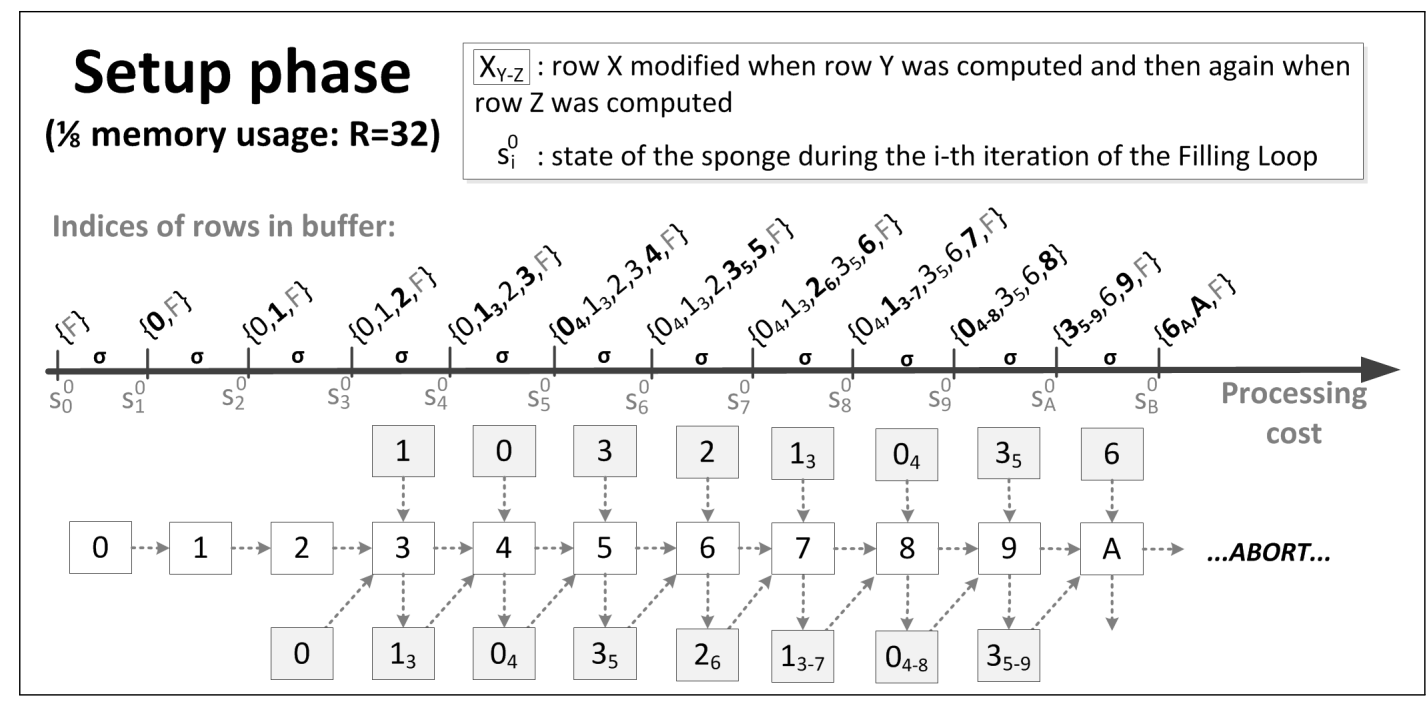

Figure 8: Attacking the Setup phase: recomputing $M\left[6_{A}\right]$ while storing $1 / 8$ of all rows and keeping $M[F]$ in memory. The most recently modified rows in each iteration are marked in bold.

In comparison, in this attack scenario we need to run $V_{1}^{0}$ while keeping rows that were originally discarded, but now need to remain in the buffer because they are used in $V_{2}^{0}$. In our example, this happens with $M\left[6_{A}\right]$, the third row from $g_{2}$ : to run $V_{1}^{0}$ with a cost of $(3 R / 4) \sigma, M\left[6_{A}\right]$ should be discarded soon after being modified (namely, after the computation of $M[B])$, thus making room for rows $\left\{M[4], M[7], M\left[2_{6}\right], M[5]\right\}$. Otherwise, $M\left[4_{C}\right]$ and $M[D]$ cannot be computed while respecting the $R / 8=4$ memory limitation. Notice that discarding $M\left[6_{A}\right]$ would not be necessary if it could be consumed in $V_{2}^{0}$ before $M\left[4_{C}\right]$ and $M[D]$, but this is not the case in this attack scenario. Therefore, to respect the $R / 8=4$ memory limitation while computing $g_{2}$, in principle the attacker would have to run $V_{1}^{0}$ twice: the first to obtain $M\left[4_{C}\right]$ and $M[D]$, which are promptly used in $V_{2}^{0}$, as well as $M[F]$, which remains in memory; and the second for computing $M\left[6_{A}\right]$ while maintaining $M[F]$ in memory so it can be consumed in $V_{2}^{0}$ right after $M\left[6_{A}\right]$. This strategy, illustrated in Figure 8, introduces an extra overhead of $11 \sigma$ to the attack in our example scenario. 
4. Finally, there is no need of computing an entire group of rows from $V_{1}^{0}$ before using those rows in $V_{2}^{0}$. For example, suppose that $M\left[0_{4-8}\right]$ and $M[9]$ are consumed by $V_{2}^{0}$ as soon as they are computed in the first re-execution of $V_{2}^{0}$. These rows can then be discarded and the attacker can use the extra space to build $g_{1}^{\prime}=\left\{M\left[2_{6-E}\right], M[B], M\left[4_{C}\right], M[D]\right\}$ with a single run of $V_{1}^{0}$. This approach should reduce the number of re-executions of $V_{1}^{0}$ and possibly alleviate the overhead from factor 3 .

\subsubsection{Storing less than what is needed: generalization}

We can generalize the discussion from section 5.1.2.3 to estimate the processing costs resulting from recursively reducing the Setup phase's memory usage by half. This can be done by imagining that any scenario with a $R / 2^{n+2}(n \geqslant 0)$ memory usage corresponds to $V_{1}^{0}$ during an attack involving half that memory.

Then, representing by $\operatorname{CostSetup}_{n}(m)$ the number of times $C L$ is executed in each window containing $m$ rows (seen as $V_{1}^{0}$ by the subsequent window) and following the same assumptions and simplifications from Section 5.1.2.3, we can write the following recursive equation:

$$
\begin{aligned}
& \operatorname{CostSetup~}_{0}(m)=3 m / 2 \quad \triangleright 1 / 4 \text { memory usage scenario }(n=0) \\
& \operatorname{CostSetup~}_{n}(m)=\stackrel{V_{1}^{0}}{\text { CostSetup }_{n-1}(m / 2)}+\stackrel{V_{2}^{0}}{r^{2}}+ \\
& \text { Re-executions of } V_{1}^{0} \\
& \underbrace{\left(3 \cdot \operatorname{CostSetup~}_{n-1}(m / 2) / 4\right) \cdot\left(2^{n+1}\right)}_{\begin{array}{c}
\text { approximate cost of } \\
\text { each execution }
\end{array}}
\end{aligned}
$$


For example, for $n=2$ (and, thus, a memory usage of $R / 16$ ), we have:

$$
\begin{aligned}
\operatorname{CostSetup~}_{2}(R)= & \operatorname{CostSetup~}_{1}(R / 2)+R / 2+\left(3 \cdot \operatorname{CostSetup}_{1}(R / 2) / 4\right) \cdot\left(2^{2+1}\right) \\
= & 7 \operatorname{CostSetup}_{1}(R / 2)+R / 2 \\
= & 7\left(\operatorname{CostSetup}_{0}(R / 4)+R / 4+\right. \\
& \left.\left(3 \cdot \operatorname{CostSetup}_{0}(R / 4) / 4\right) \cdot\left(2^{1+1}\right)\right)+R / 2 \\
= & 7(3 R / 8+R / 4+(3 \cdot(3 R / 8) / 4) \cdot 4)+R / 2 \\
= & 51 R / 4
\end{aligned}
$$

In Equation 5.1, we assume that the cost of each re-execution of $V_{1}^{0}$ can be approximated to $3 / 4$ of its total cost. We argue that this is a reasonable approximation because, as discussed in section 5.1.2.3, between $50 \%$ and $100 \%$ of $V_{1}^{0}$ needs to be executed when recovering each of the $(R / 2) /\left(R / 2^{n+2}\right)=2^{n+1}$ groups of $R / 2^{n+2}$ rows required by $V_{2}^{0}$.

The fact that Equation 5.1 assumes that only $2^{n+1}$ re-executions of $V_{1}^{0}$ are required, on the other hand, it is likely to become an oversimplification as $R$ and $n$ grow. The reason is that factor 4 discussed in section 5.1.2.3 is unlikely to compensate factor 3 in these cases. After all, as the memory available drops, it should become harder for the attacker to spare some space for rows that are not immediately needed.

The theoretical upper limit for the number of times $V_{1}^{0}$ would have to be executed during $V_{2}^{0}$ when the memory usage is $m$ would then be $m / 4$ : this corresponds to a hypothetical scenario in which, unless promptly consumed, no row required by $V_{2}^{0}$ remains in the buffer during $V_{1}^{0}$; then, since $V_{2}^{0}$ revisits rows from $V_{1}^{0}$ in an alternating pattern, approximately a pair of rows can be recovered with each execution of $V_{1}^{0}$, as the next row required is likely to have already been computed and discarded in that same execution. 
The recursive equation for estimating this upper limit would then be (in number of executions of $C L)$ :

$$
\begin{aligned}
& \operatorname{CostSetup~}_{0}(m)=3 m / 2 \quad \triangleright 1 / 4 \text { memory usage scenario }(n=0) \\
& \operatorname{CostSetup~}_{n}(m)=\stackrel{V_{1}^{0}}{\operatorname{CostSetup}_{n-1}(m / 2)}+\stackrel{V_{2}^{0}}{r / 2}+ \\
& \frac{\text { Re-executions of } V_{1}^{0}}{\underbrace{\left(3 \cdot \text { CostSetup }_{n-1}(m / 2) / 4\right) \cdot(m / 4)}_{\begin{array}{c}
\text { approximate cost of } \\
\text { each execution }
\end{array}}}
\end{aligned}
$$

The upper limit for a memory usage of $R / 16$ could then be computed as:

$$
\begin{aligned}
& \operatorname{CostSetup~}_{2}(R)=\operatorname{CostSetup~}_{1}(R / 2)+R / 2+\left(3 \cdot \operatorname{CostSetup}_{1}(R / 2) / 4\right) \cdot(R / 4) \\
& =(1+3 R / 16) \text { CostSetup }_{1}(R / 2)+R / 2 \\
& =(1+3 R / 16)\left(\text { CostSetup }_{0}(R / 4)+R / 4+\right. \\
& \left.\left(3 \cdot \operatorname{CostSetup}_{0}(R / 4) / 4\right) \cdot(R / 8)\right)+R / 2 \\
& =(1+3 R / 16)(3 R / 8+R / 4+(3 \cdot(3 R / 8) / 4) \cdot(R / 8))+R / 2 \\
& =18(R / 16)+39(R / 16)^{2}+(3 R / 16)^{3}
\end{aligned}
$$

Even though this upper limit is mostly theoretical, we do expect the $R^{n+1}$ component resulting from Equation 5.2 to become expressive and dominate the running time of Lyra2's Setup phase as $n$ grows and the memory usage drops much below $R / 2^{8}$ (i.e., for $n \gg 1$ ). In summary, these observations can be formalized in the following Conjecture:

Conjecture 2. Consider that Lyra2 operates with parameters $T, R$ and $C$. Whilst the regular memory and processing costs of its Setup phase's are, respectively, MemSetup $=R \cdot C \cdot b$ bits and CostSetup $=R \cdot \sigma$, running it with a memory cost of approximately MemSetup $/ 2^{n+2}$ leads to an average processing cost CostSetup $(R)$ that is given by recursive Equations 5.1 (for a lower bound) and 5.2 (for an upper bound). 


\subsubsection{Storing only intermediate sponge states}

Besides the strategies mentioned in the previous sections, and possibly complementing them, one can try to explore the fact that the sponge states are usually smaller than a row's cells for saving memory: while rows have $b \cdot C$ bits, a state is up to $C$ times smaller, taking $w=b+c$ bits. More precisely, by storing all sponge states, one can recompute any cell of a given row whenever it is required, rather than computing the entire row at once. For example, the initialization of each cell of $M[2]$ requires only one cell from $M[1]$. Similarly, initializing a cell of $M[4]$ takes one cell from $M[0]$, as well as one from $M[1]$ and up to two cells from $M[3]$ (one because $M[3]$ is itself fed to the sponge and another required to the computation of $\left.M\left[1_{3}\right]\right)$.

An attack that computes only one cell at a time would be easy to build if the cells sequentially output by the sponge during the initialization of $M[i]$ could be sequentially employed as input in the initialization of $M[j>i]$. Indeed, in that hypothetical case, one could build a circuitry like the one illustrated in Figure 9 to compute cells as they are required. For example, one could compute $M[2][0]$ in this scenario with (1) states $s_{0,0}^{0}, s_{1,0}^{0}$ and $s_{2,0}^{0}$, and (2) two $b$-long buffers, one for $M[0][0]$ so it can be used for computing $M[1][0]$, and the other for storing $M[1][0]$ itself, used as input for the sponge in state $s_{2,0}^{0}$. After that, the same buffers could be reused for storing $M[0][1]$ and $M[1][1]$ when computing $M[2][1]$, using the same sponge instances that are now in states $s_{0,1}^{0}, s_{1,1}^{0}$ and $s_{2,1}^{0}$. This

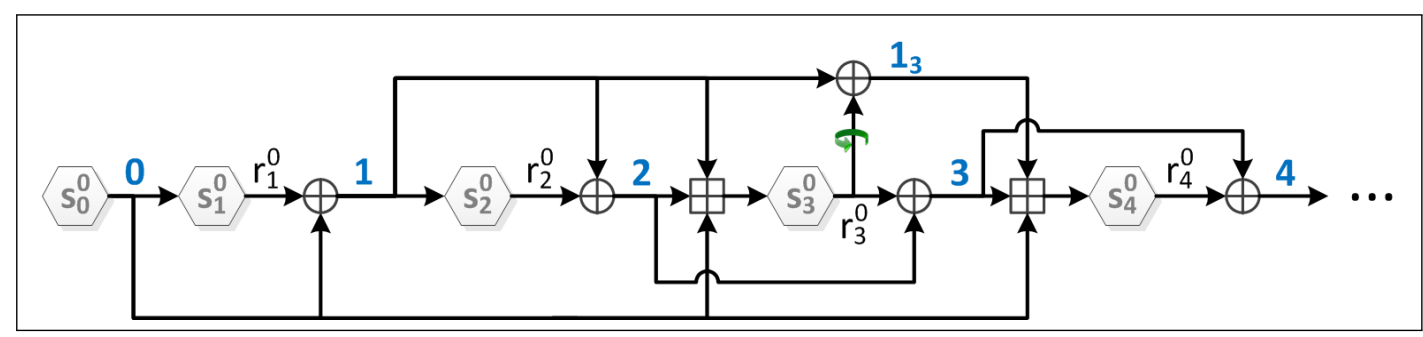

Figure 9: Attacking the Setup phase: storing only sponge states. 
process could then be iteratively repeated until the computation of $M[2][C-1]$. At that point, we would have the value of $s_{3,0}^{0}$ and could apply an analogous strategy for computing $M[3]$. The total processing cost of computing $M[2]$ would then be $3 \sigma$, since it would involve one complete execution of $C L$ for each of the sponge instances initially in states $s_{0,0}^{0}, s_{1,0}^{0}$ and $s_{2,0}^{0}$. As another example, the computation of $M[4][\mathrm{col}]$ could be performed in a similar manner, with states $s_{0,0}^{0}-s_{4,0}^{0}$ and buffers for $M[0][\mathrm{col}], M[1][\mathrm{col}]$ and $M[3][\mathrm{col}]$ (used as inputs for the sponge in state $s_{4,0}^{0}$ ), as well as for $M[2][\mathrm{col}]$ (required in the computation of $M[3][\mathrm{col}])$; the total processing cost would then be $5 \sigma$.

Generalizing this strategy, any $M[$ row $]$ could be processed using only row buffers and row +1 sponge instances in different states, leading to a cost of $r o w \cdot \sigma$ for its computation. Therefore, for the whole Setup phase, the total processing cost would be around $\left(R^{2} / 2\right) \sigma$ using approximately $2 / C$ of the memory required in a regular execution of Lyra2.

Even though this attack venue may appear promising at first sight for a large $C / R$ ratio, it cannot be performed as easily as described in the above theoretical scenario. This happens because Lyra2 reverses the order in which a row's cells are written and read, as illustrated in Figure 10. Therefore, the order in which the cells from any $M[i]$ are picked to be used as input during the initialization of $M[j>i]$ is the opposite of the order in which they are output by the sponge. Considering this constraint, suppose we want to sequentially recompute $M[1][0]$ through $M[1][C-1]$ as required (in that order) for the initialization of $M[2][C-1]$ through $M[2][0]$ during the first iteration of the Filling Loop. From the start, we have a problem: since $M[1][0]=M[0][C-1] \oplus H_{\rho} \cdot d u p l e x(M[0][C-1], b)$, its recomputation requires $M[0][C-1]$ and $s_{1, C-1}^{0}$. Consequently, computing $M[2][C-$ 1] as in our hypothetical scenario would involve roughly $\sigma$ to compute $M[0][0]$ from $s_{0,0}^{0}$. A similar issue would occur right after that, when initializing $M[2][C-2]$ 


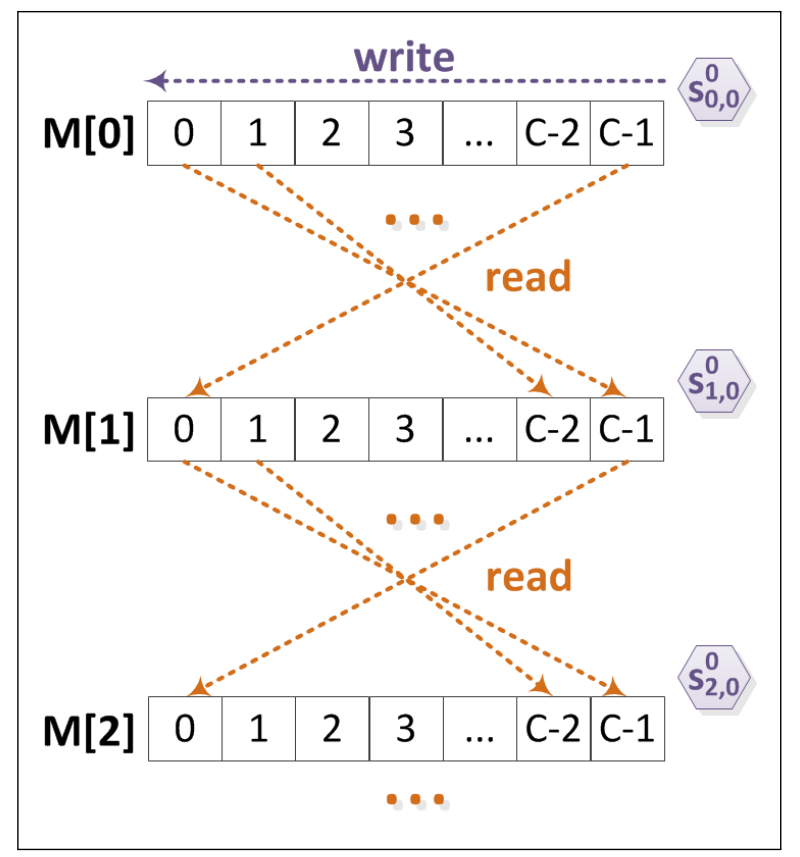

Figure 10: Reading and writing cells in the Setup phase.

from $M[1][1]$ : unless inverting the sponge's (reduced-round) internal permutation is itself easy, $M[0][1]$ cannot be easily obtained from $M[0][0]$, and neither the sponge state $s_{1, C-2}^{0}$ (required for recomputing $\left.M[1][1]\right)$ from $s_{1, C-1}^{0}$. On the other hand, recomputing $M[0][1]$ and $s_{1, C-2}^{0}$ from the values of $s_{0,1}^{0}$ and $s_{1,1}^{0}$ resulting from the previous step would involve a processing cost of approximately $(C-$ $2) \sigma / C$. If we repeat this strategy for all cells of $M[2]$, the total processing cost of initializing this row should be in the order of $C$ times higher the " $\sigma$ " obtained in our hypothetical scenario. Since the conditions for this $C$ multiplication factor appear in the computation of any other row, the processing time of this attack venue against Lyra2 is expected to become $C\left(R^{2} / 2\right) \sigma$ rather than simply $\left(R^{2} / 2\right) \sigma$, counterbalancing the memory reduction lower than $1 / C$ potentially obtained.

Obviously, one could store additional sponge states aiming at a lower processing time. For example, by storing the sponge state $s_{i, C / 2}^{0}$ in addition to $s_{i, 0}^{0}$, the attack's processing costs may be reducible by half. However, the memory cuts obtained with this approach diminish as the number of intermediate sponge states stored grow, eventually defeating the whole purpose of the attack. All 
things considered, even if feasible, this attack venue does not seem much more advantageous than the approaches discussed in the previous sections.

\subsubsection{Adding the Wandering phase: consumer-producer strategy}

During each iteration of the Wandering phase, the rows modified in the previous iteration are input to the sponge together with two other (pseudorandomly picked) rows. The latter two rows are then XORed with the sponge's output and the result is fed to the sponge in the subsequent iteration. To analyze the effects of this phase, it is useful to consider an "average", slightly simplified scenario like the one depicted in Figure 11, in which all rows are modified only once during every $R / 2$ iterations of the Visitation Loop, i.e., during $V_{1}^{1}$ the sets formed by the values assumed by row ${ }^{0}$ and by row ${ }^{1}$ are disjoint. We then apply the same principle to $V_{2}^{1}$, modifying each row only once more in a different (arbitrary) pseudorandom order. We argue that this is a reasonable simplification, given the fact that the indices of the picked rows form a uniform distribution.

In addition, we argue that this is actually beneficial for the attacker, since any row required during $V_{1}^{1}$ can be obtained simply by running the Setup phase once again, instead of involving recomputations of the Wandering phase itself. We also note that, in the particular case of Figure 11, we make the visitation order in $V_{1}^{1}$ be the exact opposite of the initialization/update of rows during $V_{2}^{0}$,

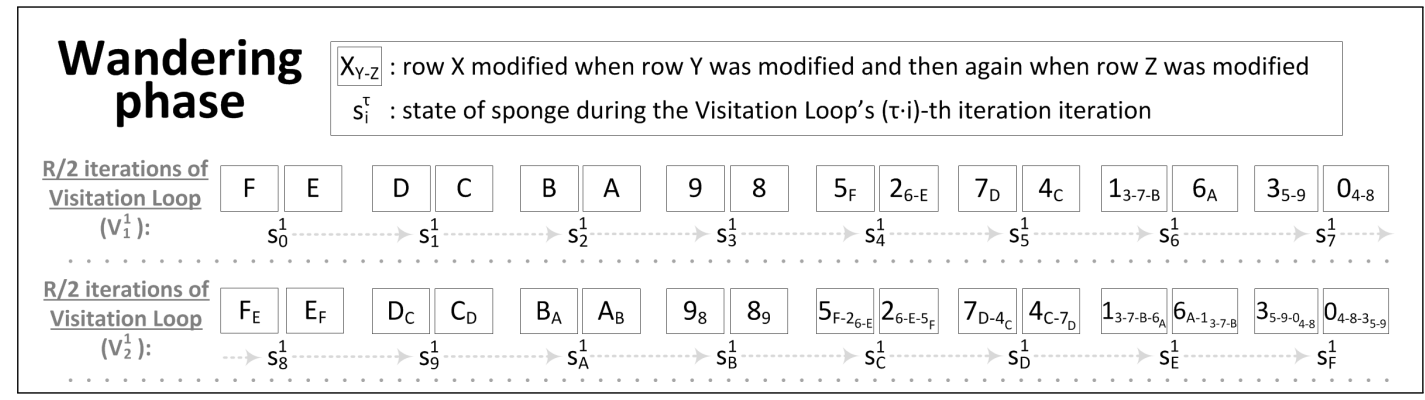

Figure 11: An example of the Wandering phase's execution. 
while in $V_{2}^{1}$ the order is the same as in $V_{1}^{1}$, for the sake of illustrating worst and best case scenarios (respectively).

In this scenario, the $R / 2$ iterations of $V_{1}^{1}$ cover the entire memory matrix. The relationship between $V_{1}^{1}$ and $V_{2}^{0}$ is, thus, very similar to that between $V_{2}^{0}$ and $V_{1}^{0}$ : if any row initialized/modified during $V_{2}^{0}$ is not available when it is required by $V_{1}^{1}$, then it is probable that the Setup phase will have to be (partially) run once again, until the point the attacker is able to recover that row. However, unlike the Setup phase, the probabilistic nature of the Wandering phase prevents the attacker from predicting which rows from $V_{1}^{1}$ can be safely discarded, which is deemed to raise the average number of re-executions of $V_{1}^{1}$. Consequently, we can adapt the arguments employed in Section 5.1.2 to estimate the cost of lowmemory attacks when the execution includes the Wandering phase, which is done in what follows for different values of $T$.

\subsubsection{The first $R / 2$ iterations of the Wandering phase with $1 / 2$ memory usage.}

We start our analysis with an attack involving only $R / 2$ rows and $T=1$. Even though this memory usage would allow the attacker to run the whole Setup phase with no penalty (see Section 5.1.2.1), the Wandering phase's Visitation Loop is not so lenient: in each iteration of $V_{1}^{1}$, there is only a $25 \%$ chance that row $^{0}$ and row $^{1}$ are both available in memory. Hence, $75 \%$ of the time the attacker will have to recompute at least one of the missing rows.

To minimize the cost of $V_{1}^{1}$ in this context, one possible strategy is to always keep in memory rows $M[i \geqslant 3 R / 4]$, using the remaining $R / 4$ memory budget as a spare for recomputations. The reasoning behind this approach is that: (1) $3 / 4$ of the Setup phase can be run with $R / 4$ without internal recomputations (see section 5.1.2.2); (2) since rows $M[i \geqslant 3 R / 4]$ are already available, this execution gives 
the updated value of any row $\in[R / 2, R[$ and of half of the rows $\in[0, R / 2[$; and (3) by XORing pairs of rows $M[i \geqslant 3 R / 4]$ accordingly, the attacker can recover any $r_{i \geqslant 3 R / 4}^{0}$ output by the sponge and, then, use it to compute the updated value of any row $\in[0, R / 2[$ from the values obtained from the first half of the Setup. In the scenario depicted by Figure 11 , for example, $M\left[5_{F}\right]$ can be recovered by computing $M[5]$ and then making $M\left[5_{F}\right][\mathrm{col}]=M[5][\mathrm{col}] \oplus \operatorname{rot}\left(r_{F}^{0}[\mathrm{col}]\right)$, where $r_{F}^{0}[\operatorname{col}]=M[F][C-1-\operatorname{col}] \oplus M[E][\operatorname{col}]$.

With this approach, recomputing rows when necessary can take from $(R / 4) \sigma$ to $(3 R / 4) \sigma$ if the Setup phase is executed just like shown in Section 5.1.2.1. It is not always necessary pay this cost for every iteration of $V_{1}^{1}$, however, if the needed row $(\mathrm{s})$ can be recovered from those already in memory. For example, if during $V_{1}^{1}$ the rows are visited in the exact same order of their initialization/update in $V_{2}^{0}$, then each row recovered can be used by $V_{1}^{1}$ before being discarded. In principle, a very lucky attacker could then be able to run the entire $V_{1}^{1}$ by executing $3 / 4$ of the Setup only once. Assuming for simplicity that the $(R / 2) \sigma$ average models a more usual scenario, the cost of each of the $R / 2$ iterations of $V_{1}^{1}$ can be estimated as: 1 in $1 / 4$ of these iterations, when row ${ }^{0}$ and row $^{1}$ are both in memory; and roughly $(R / 2) \sigma$ in $3 / 4$ of its iterations, when one or a pair of rows need to be recovered. The total cost of $V_{1}^{1}$ becomes, thus, $((1 / 4) \cdot(R / 2)+(3 / 4) \cdot(R / 2) \cdot(R / 2)) \sigma \approx$ $\left(3 R^{2} / 16\right) \sigma$.

After that, when $V_{2}^{1}$ is reached, the situation is different from what happens in $V_{1}^{1}$ : since the rows required for any iteration of $V_{2}^{1}$ have been modified during the execution of $V_{1}^{1}$, it does not suffice to (partially) run the Setup phase once again to get their values. For example, in the scenario depicted in Figure 11, the rows required for iteration $i=8$ of the Visitation Loop besides $M\left[\operatorname{prev}^{0}\right]=M[A]$ and $M\left[\right.$ prev $\left.^{1}\right]=9$ are $M\left[8_{1_{3-7-B}}\right]$ and $M\left[B_{6_{A}}\right]$, both computed during $V_{1}^{1}$. Therefore, if these rows have not been kept in memory, $V_{1}^{1}$ will have to be (partially) run once 
again, which implies new runs of the Setup itself. The cost of these re-executions are likely to be lower than originally, though, because now the attacker can take advantage of the knowledge about which rows from $V_{2}^{0}$ are needed to compute each row from $V_{1}^{1}$. On the other hand, keeping $M[i \geqslant 3 R / 4]$ is unlikely to be much advantageous now, because that would reduce the attacker's ability to bufferize rows from $V_{1}^{1}$.

In this context, one possible approach is to keep in memory the sponge's state at the beginning of $V_{1}^{1}$ (i.e., $s_{0}^{1}$ ), as well as the corresponding value of $p r e v^{0} \boxplus p r e v^{1}$ used as part of the sponge's input at this point (in our example, $M[F] \boxplus M\left[5_{F}\right]$ ). This allows the Setup and $V_{1}^{1}$ to run as different processes following a producerconsumer paradigm: the latter can proceed as long as the required inputs (rows) are provided by the former, the available memory budget being used to build their buffers. Using this strategy, the Setup needs to be run from 1 to 2 times during $V_{1}^{1}$. The first case refers to when each pair of rows provided by an iteration of $V_{2}^{0}$ can be consumed by $V_{1}^{1}$ right away, so they can be removed from the Setup's buffer similarly to what is done in Section 5.1.2.1. This happens if rows are revisited in $V_{1}^{1}$ in the same order lastly initialized/updated during $V_{2}^{0}$. The second extreme occurs when $V_{1}^{1}$ takes too long to start consuming rows from $V_{2}^{0}$, hence, some rows produced by the latter end up being discarded due to the lack of space in the Setup's buffer. This happens, for example, if $V_{1}^{1}$ revisits rows indexed by row $w^{0}$ during $V_{2}^{0}$ before those indexed by $r o w^{1}$, in the reverse order of their initialization/update, as is the case in Figure 11. Then, ignoring the fact that the Setup only starts providing useful rows for $V_{1}^{1}$ after half of its execution, on average we would have to run the Setup 1.5 times, these re-executions leading to an overhead of roughly $(3 R / 2) \sigma$.

From these observations, we can estimate that recomputing any row from $V_{2}^{1}$ would require running $50 \%$ of $V_{1}^{1}$ on average. The cost of doing so would 
be $(R / 4+3 R / 4) \sigma$, the first parcel of the sum corresponding to the cost of $V_{1}^{1}$ 's internal iterations and the second to the overhead incurred by the underlying Setup re-executions. As a side effect, this would also leave in $V_{1}^{1}$ 's buffer $R / 2$ rows, which may reveal useful during the subsequent iteration of $V_{2}^{1}$. The average cost of the $R / 2$ iterations of $V_{2}^{1}$ would then be: $\sigma$ whenever both $M\left[\right.$ row $\left.^{0}\right]$ and $M\left[\right.$ row $\left.^{1}\right]$ are available, which happens in $1 / 4$ of these iterations; roughly $R \sigma$ whenever $M\left[r_{0 w}^{0}\right]$ and/or $M\left[r_{0 w}{ }^{1}\right]$ need to be recomputed, therefore, for $1 / 4$ of these iterations. This leads to a total cost of $\left(R / 8+3 R^{2} / 8\right) \sigma$ for $V_{2}^{1}$. Adding up the cost of Setup, $V_{1}^{1}$ and $V_{2}^{1}$, the computation cost of Lyra2 when the memory usage is halved and $T=1$ can then be estimated as $R \sigma+\left(3 R^{2} / 16\right) \sigma+\left(R / 8+3 R^{2} / 8\right) \sigma \approx$ $(3 R / 4)^{2} \sigma$ for this strategy.

\subsubsection{The whole Wandering phase with $1 / 2$ memory usage}

Generalizing the discussion for all iterations of the Wandering phase, the execution of $V_{1}^{\tau}$ (resp. $V_{2}^{\tau}$ ) could use $V_{2}^{\tau-1}$ (resp. $V_{1}^{\tau}$ ) similarly to what is done in Section 5.1.3.1. Therefore, as Lyra2's execution progresses, it creates a dependence graph in the form of an inverted tree as depicted in Figure 12, level $\ell=0$ corresponding to the Setup phase and each $R / 2$ iterations of the Visitation Loop raising the tree's depth by one. Hence, the full execution of any level $\ell>0$ requires roughly all rows modified in the previous level $(\ell-1)$. With $R / 2$ rows in memory, the original computation of any level $\ell$ can then be described by the following recursive equation (in number of executions of $C L$ ):

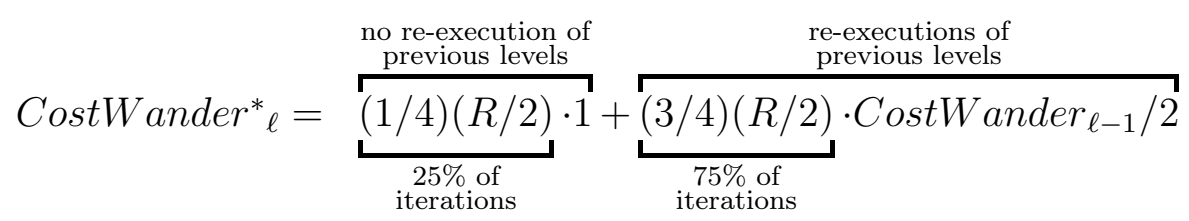




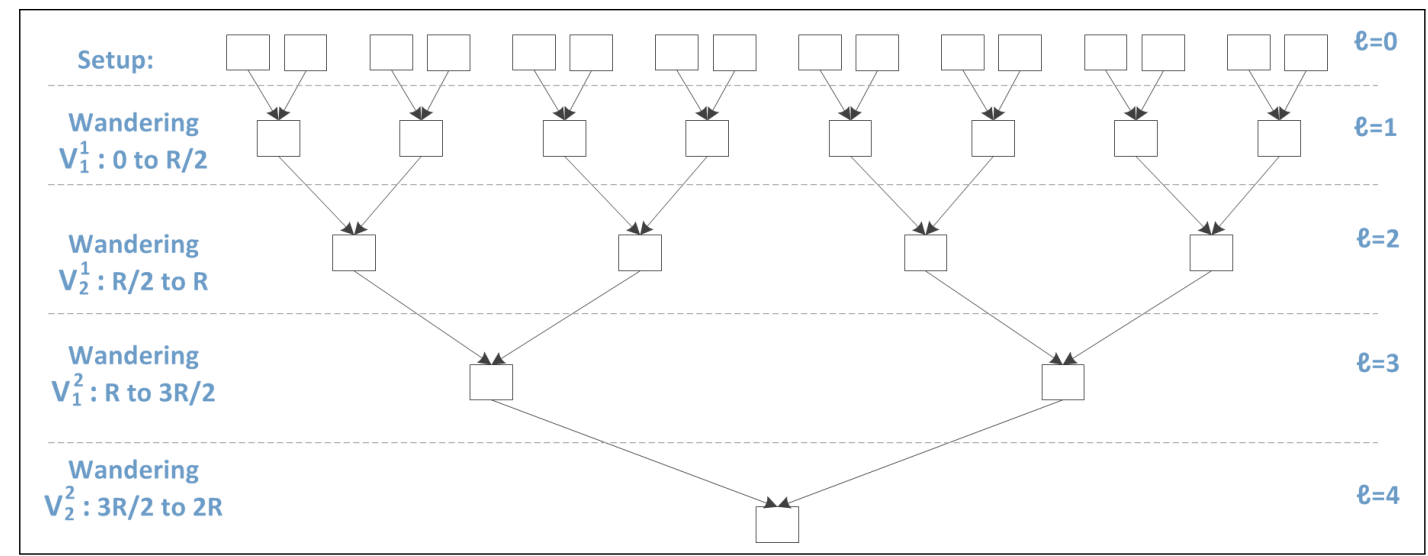

Figure 12: Tree representing the dependence among rows in Lyra2.

The value of CostWander C-1 $_{\text {in }}$ Equation 5.3 is lower than that of $\operatorname{CostWander}_{\ell-1}$, however, since the former is purely deterministic. To estimate such cost, we can use the same strategy adopted in Section 5.1.3.1: keeping the sponge's state at the beginning of each level $\ell$ and the corresponding value of $\operatorname{prev}^{0} \boxplus$ prev $^{1}$, and then running level $\ell-11.5$ times on average to recover each row that needs to be consumed. For any level $\ell$, the resulting cost can be described by the following recursive equation:

$$
\begin{aligned}
& \text { CostWander }_{0}=R \quad \triangleright \text { The Setup phase } \\
& \text { CostWander }_{\ell}=\underbrace{(3)}_{\begin{array}{c}
\text { internal } \\
\text { computations }
\end{array} / \underbrace{R / 2}_{\begin{array}{c}
\text { re-executions of } \\
\text { previous level }(\ell-1)
\end{array}}+(3 / 2) \cdot \operatorname{CostWander}{ }_{\ell-1}}=R \cdot\left(2(3 / 2)^{\ell}-1\right)
\end{aligned}
$$

Combining Equations 5.3 and 5.4 with Lemma 1, we get that the cost (in number of executions of $C L$ ) of running Lyra2 with half of the prescribed memory usage for a given $T$ would be roughly:

$$
\begin{aligned}
\operatorname{CostLyra2}_{(1 / 2)}(R, T) & =R+\text { CostWander }^{*}{ }_{1}+\cdots+\text { CostWander }^{*}{ }_{2 T} \\
& =(T+4) \cdot(R / 4)+\left(3 R^{2} / 4\right) \cdot\left((3 / 2)^{2 T}-(T+2) / 2\right) \\
& =O\left((3 / 2)^{2 T} R^{2}\right)
\end{aligned}
$$




\subsubsection{The whole Wandering phase with less than $1 / 2$ memory usage}

A memory usage of $1 / 2^{n+2}(n \geqslant 0)$ is expected to have three effects on the execution of the Wandering phase. First, the probability that row $w^{0}$ and $r o w^{1}$ will both be available in memory at any iteration of the Visitation Loop drops to $1 / 2^{n+2}$, meaning that Equation 5.3 needs to be updated accordingly. Second, the cost of running the Setup phase is deemed to become higher, its lower and upper bounds being estimated by Equations 5.1 and 5.2, respectively. Third, level $\ell-1$ may have to be re-executed $2^{n+2}$ times to allow the recovery of all rows required by level $\ell$, which has repercussions on Equation 5.4: on average, CostWander ${ }_{\ell}$ will involve $\left(1+2^{n+2}\right) / 2 \approx 2^{n+1}$ calls to CostWander ${ }_{\ell-1}$.

Combining these observations, we arrive at

$$
\begin{aligned}
& \operatorname{CostWander}_{\ell, n}^{*}=\frac{\begin{array}{c}
\text { no re-execution of } \\
\text { previous levels }
\end{array}}{\frac{(R / 2) \cdot\left(1 / 2^{n+2}\right)}{1 / 2^{n+2} \text { of iterations }} \cdot 1}+ \\
& \text { re-executions of } \\
& \text { previous levels } \\
& \frac{(R / 2) \cdot\left(1-1 / 2^{n+2}\right) \cdot\left(\text { CostW }_{\text {all other iterations }}\right.}{\left(R \text { and }_{\ell-n}\right) / 2}
\end{aligned}
$$

as an estimate for the original (probabilistic) executions of level $\ell$, and at

$$
\begin{aligned}
& \operatorname{CostWander}_{0, n}=\operatorname{CostSetup}_{n}(R) \quad \triangleright \text { The Setup phase }
\end{aligned}
$$

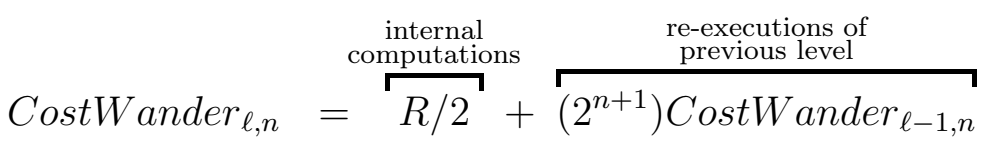

$$
\begin{aligned}
& =(R / 2) \cdot\left(1-\left(2^{n+1}\right)^{\ell}\right) /\left(1-2^{n+1}\right)+\left(2^{n+1}\right)^{\ell} \cdot \operatorname{CostSetup}_{n}(R)
\end{aligned}
$$

for the deterministic re-executions of level $\ell$.

Equations 5.6 and 5.7 can then be combined to provide the following estimate 
to the total cost of an attack against Lyra2 involving $R / 2^{n+2}$ rows instead of $R$ :

$$
\begin{aligned}
\operatorname{CostLyra2}_{\left(1 / 2^{n+2}\right)}(R, T)= & \text { CostSetup }_{n}(R)+\operatorname{CostWander}_{1, n}^{*}+\cdots+ \\
& \left.\operatorname{CostWander}_{2 T, n}\right) \sigma \\
\approx & O\left(\left(R^{2}\right)\left(2^{2 n T}\right)+R \cdot \operatorname{CostSetup~}_{n}(R) \cdot 2^{2 n T}\right)
\end{aligned}
$$

Since, as suggested in Section 5.1.2.4, the upper bound CostSetup $_{n}=$ $O\left(R^{n+1}\right)$ given by Equation 5.2 is likely to become a better estimate for CostSetup $_{n}$ as $n$ grows, we conjecture that the processing cost of Lyra2 using the strategy hereby discussed is actually $O\left(2^{2 n T} R^{n+2}\right)$ for $n \gg 1$.

\subsubsection{Adding the Wandering phase: sentinel-based stra- tegy}

The analysis of the consumer-producer strategy described in Section 5.1.3 shows that updating many rows in the hope they will be useful in an iteration of the Wandering phase's Rows Loop does reduce the attack cost by too much, since these rows are only useful $25 \%$ of the time; in addition, it has the disadvantage of discarding the rows initialized/updated during VLoop10, which are certainly required $75 \%$ of the time. From these observations, we can consider an alternative strategy that employs the following trick ${ }^{1}$ : if we keep in memory all the rows produced during $V_{1}^{0}$ and a few rows initialized during $V_{2}^{0}$ together with the corresponding sponge states, we can skip part of the latter's iterations when initializing/updating the rows required by $V_{1}^{1}$. In our example scenario, we would keep in memory rows $M\left[0_{4}\right]-M[7]$ as output by $V_{1}^{0}$. Then, by keeping rows $M[C]$ and $M\left[4_{C}\right]$ in memory together with state $s_{D}^{0}, M[D]$ and $M\left[7_{D}\right]$ can be recomputed directly from $M[7]$ with a cost of $\sigma$, while $M[F]$ and $M\left[5_{F}\right]$ can be recovered with a cost of $3 \sigma$. In both cases, $M[C]$ and $M\left[4_{C}\right]$ act as "sentinels"

\footnotetext{
${ }^{1}$ This is analogous to the attack presented in (KHOVRATOVICH; BIRYUKOV; GROBSCHÄDL, 2014) for the version of Lyra2 originally submitted to the Password Hashing Competition as "V1"
} 
that allow us to skip the computation of $M[8]-M[C]$.

More generally, suppose we keep rows $M[0 \leqslant i<R / 2]$, obtained by running $V_{1}^{0}$, as well as $\epsilon>0$ sentinels equally distributed in the range $[R / 2, R[$. Then, the cost of recovering any row output by $V_{2}^{0}$ would range from 0 (for the sentinels themselves) to $(R / 2 \epsilon) \sigma$ (for rows the farthest away from the sentinels), or $(R / 4 \epsilon) \sigma$ on average. The resulting memory cost of such a strategy is approximately $R / 2$ (for the rows from $V_{1}^{0}$ ), plus $2 \epsilon$ (for the fixed sentinels), plus 2 (for storing the value of $p r e v^{0}$ and $\operatorname{prev}^{1}$ while computing a given row inside the area covered by a fixed sentinel). When compared with the consumer-produces approach, one drawback is that only the $2 \epsilon$ rows acting as sentinels can be promptly consumed by $V_{1}^{1}$, since rows provided by $V_{1}^{0}$ are overwritten during the execution of $V_{2}^{0}$. Nonetheless, the average cost of $V_{1}^{1}$ ends up being approximately $(R / 2) \cdot(R / 4 \epsilon) \sigma$ for a small $\epsilon$, which is lower than in the previous approach for $\epsilon \geqslant 2$. With $\epsilon=R / 32$ sentinels (i.e., $R / 16$ rows), for example, the processing cost of $V_{1}^{1}$ would be $4 R$ for a memory usage less than $10 \%$ above $R / 2$.

We can then employ a similar trick for the execution of $V_{2}^{1}$, by placing sentinels along the execution of $V_{1}^{1}$ to reduce the cost of the latter's recomputations. For instance, $M\left[9_{8}\right]$ and $M\left[8_{9}\right]$ could be used as sentinels to accelerate the recovery of rows visited in the second half of $V_{1}^{1}$ in our example scenario (see Figure 11). However, in this case the sentinels are likely to be less effective. The reason is that the steps taken from each sentinel placed in $V_{1}^{1}$ should cover different portions of $V_{2}^{0}$, obliging some iterations of $V_{2}^{0}$ to be executed. For example, using the same $\epsilon=R / 32$ sentinels as before to keep the memory usage near $R / 2$, we could distribute half of them along $V_{2}^{0}$ and the other half along $V_{1}^{1}$, so each would be covered by $\epsilon^{\prime}=\epsilon / 2$ sentinels. As a result, any row output by $V_{1}^{1}$ or $V_{2}^{0}$ could be recovered with $R / 4\left(\epsilon^{\prime}\right)=16$ executions of $C L$ on average. Unfortunately for the attacker, though, any iteration of $V_{2}^{1}$ takes two rows from $V_{1}^{1}$, which means that 
$2 \cdot 16=32$ iterations of $V_{1}^{1}$ are likely to be executed and, hence, that roughly $2 \cdot 32=64$ rows from $V_{2}^{0}$ should be required. If all of those 64 rows fall into areas covered by different sentinels placed at $V_{2}^{0}$, the average cost when computing any row from $V_{2}^{1}$ would be approximately $64 \cdot 16=1024$ executions of $C L$. In this case, the cost of the $R / 2$ iterations of $V_{2}^{1}$ would become roughly $(1024 R / 2) \sigma$ on average. This is lower than the $\approx\left(R^{2} / 2\right) \sigma$ obtained with the consumer-producer strategy for $R>1024$, but still orders of magnitude more expensive than a regular execution with a memory usage of $R$.

Obviously, two or more of the 64 rows required from $V_{2}^{0}$ may fall in the area covered by a same sentinel, which allows for a lower number of executions if the attacker computes those rows in a single sweep and keep them in memory until they are required. Even though this approach is likely to raise the attack's memory usage, it would lead to a lower processing cost, since any part of $V_{2}^{0}$ covered by a same sentinel would be run only once during any iteration of $V_{2}^{1}$. However, if the number of sentinels in $V_{2}^{0}$ is large in comparison with the number of rows required by each of $V_{2}^{1}$ 's iteration (i.e., for $\epsilon / 2 \gg 64$, which implies $R \gg 8192$ ), we can ignore such "sentinel collisions" and the average cost described above should hold. This should also be the cost obtained if the attacker prefers not to raise the attack's memory usage when collisions occur, but instead recomputes rows that can be obtained from a given sentinel by running the same part of $V_{2}^{0}$ more than once.

For the sake of completeness, it is interesting to analyze such memoryprocessing tradeoffs for dealing with collisions when the cost of this sentinel-based strategy starts to get higher than the one obtained with the consumer-producer strategy. Specifically, for $R=1024$ this strategy is deemed to create many sentinel collisions, with each of the $\epsilon^{\prime}=16$ sentinels placed along $V_{2}^{0}$ being employed for recomputing roughly $64 / 16=4$ out of the 64 rows from $V_{2}^{0}$ required by each 
iteration of $V_{2}^{1}$. In this scenario, the 4 rows under a same sentinel's responsibility can be recovered in a single sweep and then stored until needed. Assuming that those 4 rows are equally distributed over the corresponding sentinel's coverage area, the average cost of the executions related to that sentinel would then be $(7 / 8)(R / 2) /(\epsilon / 2)=28 \sigma$. This leads to $16 \cdot 28 \sigma=448 \sigma$ for all 16 partial runs of $V_{2}^{0}$, and consequently to $(448 R / 2) \sigma$ for the whole $V_{2}^{1}$. In terms of memory usage, the worst case scenario from the attacker's perspective refers to when the rows computed last from each sentinel are the first ones required during $V_{2}^{1}$, meaning that recovering 1 row that is immediately useful leaves in memory 3 that are not. This situation would lead to a storage of $3(\epsilon / 2)=3 R / 64$ rows, which corresponds to $75 \%$ of the $R / 16$ rows already employed by the attack besides the $R / 2$ base value.

As a last remark, notice that the 64 rows from $V_{2}^{0}$ can all be recovered in parallel, using 64 different processing cores, the same applying to the 2 rows from $V_{1}^{1}$, with 2 extra cores. The average cost of $V_{2}^{1}$ as perceived by the attacker would then be roughly $(16+16)(R / 2) \sigma$, which corresponds to a parallel execution of $V_{2}^{0}$ followed by a parallel execution of $V_{1}^{1}$. In this case, however, the memory usage would also be slightly higher: since each of the 66 threads would have to be associated to its own $p r e v^{0}$ and $p r e v^{1}$, the attack would require an additional memory usage of 132 rows.

\subsubsection{On the (low) scalability of the sentinel-based strategy}

Even though the sentinel strategy shows promise in some scenarios, it has low scalability for values of $T$ higher than 1 . The reason is that, as $T$ grows, the computation of any given row depends on rows recomputed from an exponentially large number of sentinels. This is more easily observed if we analyze the dependence graph depicted in Figure 13 for $T=2$, which shows the number 


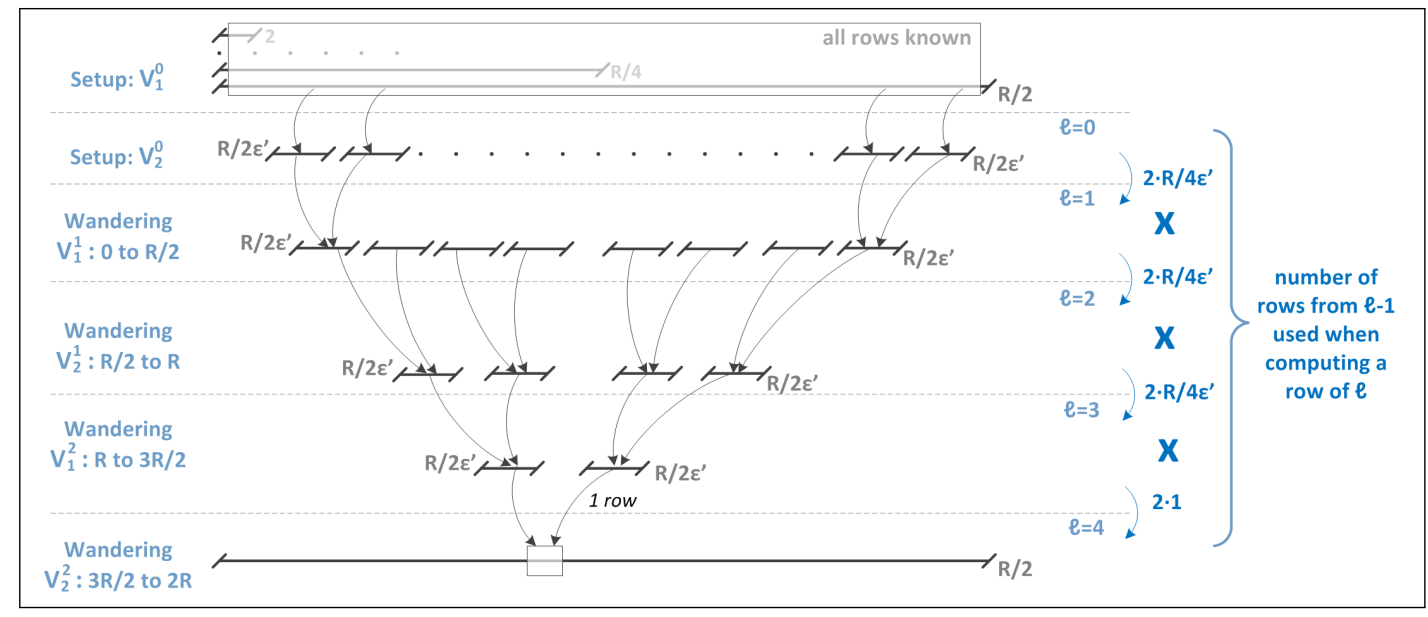

Figure 13: Tree representing the dependence among rows in Lyra2 with $T=2$ : using $\epsilon^{\prime}$ sentinels per level.

of rows from level $\ell-1$ that are needed in the sentinel-based computation of level $\ell$. In this scenario, if we assume that the $\epsilon$ sentinels are distributed along $V_{2}^{0}, V_{1}^{1}, V_{2}^{1}$ and $V_{1}^{2}$ (levels $\ell=0$ to 3 , respectively), each level will get $\epsilon^{\prime}=\epsilon / 4$ sentinels, being divided in $R / 2 \epsilon^{\prime}$ areas. As a result, even though computing a row from level $\ell=4$ takes only 2 rows from level $\ell=3$, computing a row from level $\ell<4$ involves roughly $R / 4 \epsilon^{\prime}$ iterations of that level, those iterations requiring $2\left(R / 4 \epsilon^{\prime}\right)$ rows from level $\ell-1$. Therefore, any iteration of $V_{2}^{2}$ is expected to involve the computation of $2^{4}\left(R / 4 \epsilon^{\prime}\right)^{3}$ rows from $V_{2}^{0}$, which translates to $2^{19}$ rows for $\epsilon=R / 32$. If each of these rows is computed individually, with the usual cost of $\left(R / 4 \epsilon^{\prime}\right) \sigma$ per row, the recomputations related to sentinels from $V_{2}^{0}$ alone would take $2^{19}\left(R / 4 \epsilon^{\prime}\right) \sigma=2^{24} \cdot \sigma$, leading to a cost higher than $\left(2^{24} \cdot R / 2\right) \sigma$ for the whole $V_{2}^{2}$.

More generally, for arbitrary values of $T$ and $\epsilon=R / \alpha$ (and, hence, $\epsilon^{\prime}=\epsilon / 2 T$ ), the recomputations in $V_{2}^{0}$ for each iteration of $V_{2}^{T}$ would take $2^{2 T} \cdot\left(R / 4 \epsilon^{\prime}\right)^{2 T} \sigma$, so the cost of $V_{2}^{T}$ itself would become $(\alpha \cdot T)^{2 T}(R / 2) \sigma$. Depending on the parameters employed, this cost may be higher than the $O\left((3 / 2)^{2 T} R^{2}\right)$ obtained with the consumer-producer strategy, making the latter a preferred attack venue. This is the case, for example, when we have $\alpha=32$, as in all the previous examples, 
$R \leqslant 2^{20}$, as in all the benchmarks presented in Section 6 , and $T \geqslant 2$.

Once again, attackers may counterbalance this processing cost with the temporary storage of rows that can be recomputed from a same sentinel, or of a same row that is required multiple times during the attack. However, the attackers' ability of doing so while keeping the memory usage around $R / 2$ is limited by the fact that this sentinel-based strategy commits a huge part of the attack's memory budget to the storage of all rows from $V_{1}^{0}$. Diverting part of this budget to the temporary storage of rows, on the other hand, is similar to what is done in the consumer-producer strategy itself, so that the latter can be seen as an extreme case of this approach.

On the other extreme, the memory budget could be diverted to raise the number of sentinels and, thus, reduce $\alpha$. As a drawback, the attack would have to deal with a dependence graph displaying extra layers, since then $V_{1}^{0}$ would not be fully covered. This would lead to a higher cost for the computation of each row from $V_{2}^{0}$, counterbalancing to some extent the gains obtained with the extra sentinels. For example, suppose the attacker (1) stores only $R / 4$ out of the $R / 2$ rows from $V_{1}^{0}$, using the remainder budget of $R / 4$ rows to make $\epsilon=R / 8$ sentinels, and then (2) places $\epsilon^{*}=R / 32$ sentinels (i.e., $R / 16$ rows) along the part of $V_{1}^{0}$ that is not covered anymore, thus keeping the total memory usage at $R / 2+R / 16$ rows as in the previous examples. In this scenario, the number of rows from $V_{2}^{0}$ involved in each iteration of $V_{2}^{2}$ should drop to $2^{4}\left(R / 4 \epsilon^{\prime}\right)^{3}=2^{13}$ if we assume once again that the sentinels are equally distributed through all levels (i.e., for $\epsilon^{\prime}=\epsilon / 4$ ). However, recovering a row from $V_{2}^{0}$ should not take only $R / 4 \epsilon^{\prime}=2^{3}$ executions of $C L$ anymore, but roughly $\left(R / 4 \epsilon^{\prime}\right) \cdot\left(R / 4 \epsilon^{*}\right)=2^{5}$ due to the recomputations of rows from $V_{1}^{0}$. The processing cost for the whole $V_{2}^{2}$ would then be $\left(2^{18} \cdot R / 2\right) \sigma$, which still is not lower than what is obtained with the consumer-producer strategy for $R \leqslant 2^{17}$. 
The low scalability of the sentinel-based strategy also impairs attacks with a memory usage lower than $R / 2$, since then the number of sentinels and coverage of rows from $V_{1}^{0}$ would both drop. The same scalability issues apply to attempts of recovering all rows from $V_{2}^{0}$ in parallel using different processing cores, as suggested at the end of Section 5.1.4, given that the number of cores grows exponentially with $T$.

\subsection{Slow-Memory attacks}

When compared to low-memory attacks, providing protection against slowmemory attacks is a more involved task. This happens because the attacker acts approximately as a legitimate user during the algorithm's operation, keeping in memory all information required. The main difference resides on the bandwidth and latency provided by the memory device employed, which ultimately impacts the time required for testing each password guess.

Lyra2, similarly to scrypt, explores the properties of low-cost memory devices by visiting memory positions following a pseudorandom pattern during the Wandering phase. In particular, this strategy increases the latency of intrinsically sequential memory devices, such as hard disks, especially if the attack involves multiple instances simultaneously accessing different memory sections. Furthermore, as discussed in Section 4.5, this pseudorandom pattern combined with a small $C$ parameter may also diminish speedups obtained from mechanisms such as caching and prefetching, even when the attacker employs (low-cost) randomaccess memory chips. Even though this latency may be (partially) hidden in a parallel attack by prefetching the rows needed by one thread while another thread is running, at least the attacker would have to pay the cost of frequently changing the context of each thread. We notice that this approach is particularly harmful against older model GPUs, whose internal structure were usually optimized 
toward deterministic memory accesses to small portions of memory (NVIDIA, 2014, Sec. 5.3.2).

When compared with scrypt, a slight improvement introduced by Lyra2 against such attacks is that the memory positions are not only repeatedly read, but also written. As a result, Lyra2 requires data to be repeatedly moved up and down the memory hierarchy. The overall impact of this feature on the performance of a slow-memory attack depends, however, on the exact system architecture. For example, it is likely to increase traffic on a shared memory bus, while caching mechanisms may require a more complex circuitry/scheduling to cope with the continuous flow of information from/to a slower memory level. This high bandwidth usage is also likely to hinder the construction of high-performance dedicated hardware for testing multiple passwords in parallel.

Another feature of Lyra2 is the fact that, during the Wandering phase, the columns of the most recently updated rows $\left(M\left[\right.\right.$ prev $\left.^{0}\right]$ and $M\left[\right.$ prev $\left.\left.^{0}\right]\right)$ are read in a pseudorandom manner. Since these rows are expected to be in cache during a regular execution of Lyra2, a legitimate user that configures $C$ adequately should be able to read these rows approximately as fast as if they were read sequentially. An attacker using a platform with a lower cache size, however, should experience a lower performance due to cache misses. In addition, this pseudorandom pattern hinders the creation of simple pipelines in hardware for visiting those rows: even if the attacker keeps all columns in fast memory to avoid latency issues, some selection function will be necessary to choose among those columns on the fly.

Finally, in Lyra2's design the sponge's output is always XORed with the value of existing rows, preventing the memory positions corresponding to those rows from becoming quickly replaceable. This property is, thus, likely to hinder the attacker's capability of reusing those memory regions in a parallel thread.

Obviously, all features displayed by Lyra2 for providing protection against 
slow-memory attacks may also impact the algorithm's performance for legitimate

user. After all, they also interfere with the legitimate platform's capability of taking advantage of its own caching and pre-fetching features. Therefore, it is of utmost importance that the algorithm's configuration is optimized to the platform's characteristics, considering aspects such as the amount of RAM available, cache line size, etc. This should allow Lyra2's execution to run more smoothly in the legitimate user's machine while imposing more serious penalties to attackers employing platforms with distinct characteristics.

\subsection{Cache-timing attacks}

A cache-timing attack is a type of side-channel attack in which the attacker is able to observe a machine's timing behavior by monitoring its access to cache memory (e.g., the occurrence of cache-misses) (FORLER; LUCKS; WENZEL, 2013; BERNSTEIN, 2005). This class of attacks has been shown to be effective, for example, against certain implementations of the Advanced Encryption Standard (AES) (NIST, 2001a) and RSA (RIVEST; SHAMIR; ADLEMAN, 1978), allowing the recovery of the secret key employed by the algorithms (BERNSTEIN, 2005; PERCIVAL, 2005).

In the context of password hashing, cache-timing attacks may be a threat against memory-hard solutions that involve operations for which the memory visitation order depends on the password. The reason is that, at least in theory, a spy process that observes the cache behavior of the correct password may be able to filter passwords that do not match that pattern after only a few iterations, rather than after the whole algorithm is run (FORLER; LUCKS; WENZEL, 2013). Nevertheless, cache-timing attacks are unlikely to be a matter of great concern in scenarios where the PHS runs in a single-user scenario, such as in local authentication or in remote authentications performed in a dedicated server: after 
all, if attackers are able to insert such spy process into these environments, it is quite possible they will insert a much more powerful spyware (e.g., a keylogger or a memory scanner) to get the password more directly.

On the other hand, cache-timing attacks may be an interesting approach in scenarios where the physical hardware running the PHS is shared by processes of different users, such as virtual servers hosted in a public cloud (RISTENPART et al., 2009). This happens because such environments potentially create the required conditions for making cache-timing measurements (RISTENPART et al., 2009), but are expected to prevent the installation of a malware powerful enough to circumvent the hypervisor's isolation capability for accessing data from different virtual machines.

In this context, the approach adopted in Lyra2 is to provide resistance against cache-timing attacks only during the Setup phase, in which the indices of the rows read and written are not password-dependent, while the Wandering and Wrapup phases are susceptible to such attacks. As a result, even though Lyra2 is not completely immune to cache-timing attacks, the algorithm ensures that attackers will have to run the whole Setup phase and at least a portion of the Wandering phase before they can use cache-timing information for filtering guesses. Therefore, such attacks will still involve a memory usage of at least $R / 2$ rows or some of the time-memory trade-offs discussed along Section 5.1.

The reasoning behind this design decision of providing partial resistance to cache-timing attacks is threefold. First, as discussed in Section 5.2, making password-dependent memory visitations is one of the main defenses of Lyra2 against slow-memory attacks, since it hinders caching and pre-fetching mechanisms that could accelerate this threat. Therefore, resistance against low-memory attacks and protection against cache-timing attacks are somewhat conflicting requirements. Since low- and slow-memory attacks are applicable to a wide range 
of scenarios, from local to remote authentication, it seems more important to protect against them than completely preventing cache-timing attacks.

Second, for practical reasons (namely, scalability) it may be interesting to offload the password hashing process to users, distributing the underlying costs among client devices rather than concentrating them on the server, even in the case of remote authentication. This is the main idea behind the server-relief protocol described in (FORLER; LUCKS; WENZEL, 2013), according to which the server sends only the salt to the client (preferably using a secure channel), who responds with $x=\operatorname{PHS}(p w d$, salt $)$; then, the server only computes locally $y=H(x)$ and compares it to the value stored in its own database. The result of this approach is that the server-side computations during authentication are reduced to executing one hash, while the memory- and processing-intensive operations involved in the password hashing process are performed by the client, in an environment in which cache-timing is probably a less critical concern.

Third, as discussed in (MOWERY; KEELVEEDHI; SHACHAM, 2012), recent advances in software and hardware technology may (partially) hinder the feasibility of cache-timing and related attacks due to the amount of "noise" conveyed by their underlying complexity. This technological constraint is also reinforced by the fact that security-aware cloud providers are expected to provide countermeasures against such attacks for protecting their users, such as (see (RISTENPART et al., 2009) for a more detailed discussion): ensuring that processes run by different users do not influence each other's cache usage (or, at least, that this influence is not completely predictable); or making it more difficult for an attacker to place a spy process in the same physical machine as security-sensitive processes, especially processes related to user authentication. Therefore, even if these countermeasures are not enough to completely prevent such attacks from happening, the added complexity brought by them may be enough to force the 
attacker to run a large portion of the Wandering phase, paying the corresponding costs, before a password guess can be reliably discarded.

\subsection{Garbage-Collector attacks}

A garbage-collector attack is another type of side-channel attack, in which the attacker has access to the internal memory of the target's machine after a legitimate password hashing process is finished (FORLER et al., 2014). In this context, the goal of the attacker is to find a valid password candidate based on the collected data, instead of trying all the possibilities (brute-force attack), exploiting the machine's memory management mechanisms as applied to the PHS's internal state and/or password-dependent values written in memory (FORLER et al., 2014).

Specifically in the context of password hashing, garbage-collector attacks are a threat especially against memory-hard solutions that do not overwrite its internal memory after filling it with password-dependent data. In this case, the memory parts that may have been written on disk (e.g., due to memory swapping) or are still in RAM leave a useful trace that can be used in the discovery of the original password: attackers can execute the password hashing over candidate passwords until they obtain the same memory section resulting from the correct password; if the contents of those regions match, then the password is probably correct and the whole hashing process should proceed until the end; otherwise, the test can be aborted earlier.

Albeit interesting and potentially powerful, the usefulness of such attacks against memory-hard solutions that overwrite its internal memory is quite limited. After all, when the memory visitation and overwriting is password-dependent, as in the case of Lyra2, the attacker cannot determine with a reasonable degree 
of certainty how many times a given memory region has been overwritten until a given point of the processing. Hence, even if some specific memory region is available due to swapping, it is not easy to determine when the corresponding value was obtained unless the entirety of the algorithm is run, which prevents the early abortion of the test. Therefore, since Lyra2 overwrites the sponge state multiple times and rewrites the memory matrix's rows and columns again and again, garbage-collector attacks do not apply to the algorithm, a claim that is corroborated by third-party analysis (FORLER et al., 2014).

\subsection{Security analysis of BlaMka.}

Whereas the previous analysis does not depend on the underlying sponge, it is also important to evaluate the security of BlaMka as opposed to Blake2b, especially considering that the latter has been intensively analyzed during the SHA-3 competition. Fortunately, in terms of security, a preliminary analysis of the $\operatorname{tls}(x, y)=x+y+2 \cdot \operatorname{lsw}(x) \cdot \operatorname{lsw}(y)$ operation adopted in BlaMka's $G_{\mathrm{tl} s}$ permutation indicates that its diffusion capability is at least as high as that provided by the simple word-wise addition employed by Blake2b's original $G$ function. This observation comes from the assessment of XOR-differentials over tls, defined in (AUMASSON; JOVANOVIC; NEVES, 2015) as:

Definition 1. Let $f: \mathbb{F}_{2}^{2 n} \mapsto \mathbb{F}_{2}^{n}$ be a vector Boolean function and let $\alpha, \beta$ and $\gamma$ be $n$-bit sized XOR-differences. We call $(\alpha, \beta) \mapsto \gamma$ a XOR-differential of $f$ if there exist $n$-bit strings $x$ and $y$ that satisfy $f^{\prime}(x \oplus \alpha, y \oplus \beta)=f^{\prime}(x, y) \oplus \gamma$. Otherwise, if no such $n$-bit strings $x$ and $y$ exist, we call $(\alpha, \beta) \mapsto \gamma$ an impossible $X O R$-differential of $f$.

Specifically, conducting an exhaustive search for $n=8$, we found 4 XORdifferentials that hold for all 65536 pairs $(x, y)$, both for tls and for the addition 
operation: $(0 x 00,0 x 00) \mapsto 0 x 00,(0 x 80,0 x 80) \mapsto 0 x 00,(0 x 00,0 x 80) \mapsto 0 x 80$, and $(0 x 80,0 x 00) \mapsto 0 x 80$ (in hexadecimal notation). Hence, the most common XOR-differentials have the same probability for both tls and regular additions.

However, when we analyze the XOR-differentials with second highest probability, we observe that the addition operation displays 168 XOR-differentials that hold for $50 \%$ of all $(x, y)$ pairs, while the tls operation hereby described has only 48 of such XOR-differentials. XOR-differentials with lower, but still high probabilities are also less frequent for tls than for an addition - e.g., 288 instead of 3024 differentials that hold for $25 \%$ of all $(x, y)$ pairs, - although the former displays differentials with probabilities that do not appear in the latter - e.g., 12 differentials that hold for 19200 out of the $65536(x, y)$ pairs, the third highest differential probability for tls.

Therefore, although tests with a larger number of bits would be required to confirm this analysis, by adopting the multiplication-hardened tls operation as part of its underlying $G_{\mathrm{tls}}$ permutation, BlaMka is expected to be at least as secure as Blake2b when it comes to its diffusion capability. Interestingly, given the similarity between $G_{\mathrm{t} 1 \mathrm{~s}}$ and NORX's own structure, it is quite possible that analyses of this latter scheme can also apply to the construction hereby described.

\subsection{Summary}

This chapter provided a security analysis of Lyra2, showing how it's design thwarts different attack strategies. Table 2 summarizes the discussion, providing a comparison with other relevant Password Hashing Schemes available in the literature and discussed in Section 3. We note that, among the results appearing in this table, only the data related to Lyra2 were actually analyzed and presented in this work, while the remainder of the data shown were collec- 


\begin{tabular}{|c|c|c|c|c|c|}
\hline Algorithm & $\begin{array}{c}\text { Time-Memory trade-offs } \\
\text { TMTO }\end{array}$ & $\begin{array}{c}\text { Slow } \\
\text { Memory }\end{array}$ & $\begin{array}{c}\text { Side } \\
\text { Channel }\end{array}$ & $\begin{array}{l}\text { Hardware } \\
\text { and GPUs }\end{array}$ & $\begin{array}{l}\text { Garbage } \\
\text { Collector }\end{array}$ \\
\hline Argon2i & $\begin{array}{l}\text { for } R^{\prime}=R / 6 \\
\approx 2^{15.5} \cdot T \cdot R\end{array}$ & - & $\checkmark$ & $\checkmark$ & $\checkmark$ \\
\hline Argon2d & $\begin{array}{l}\text { for } R^{\prime}=R / 6 \\
\approx 2^{19.6} \cdot T \cdot R\end{array}$ & $\checkmark$ & $x$ & $\checkmark$ & $\checkmark$ \\
\hline battcrypt & - & $\checkmark$ & $x$ & $\checkmark$ & $\checkmark$ \\
\hline Catena & $\begin{array}{c}O(1) \\
\Theta\left(R^{T+1}\right)\end{array}$ & - & $\checkmark$ & $\checkmark$ & $\checkmark$ \\
\hline Lyra & $\begin{array}{c}O(1) \\
O\left(R^{T+1}\right)\end{array}$ & $\checkmark$ & $x$ & $\checkmark$ & $\checkmark$ \\
\hline Lyra2 [ours] & $\begin{array}{c}\text { for } R^{\prime}=R / 2^{n+2}, \text { where } n \geq 0 \\
O\left(2^{2 n T} R^{2+n}\right), \text { for } n \gg 1\end{array}$ & $\checkmark$ & $!$ & $\checkmark$ & $\checkmark$ \\
\hline POMELO & - & $\checkmark$ & $!$ & $\checkmark$ & $\checkmark$ \\
\hline yescrypt & $\begin{array}{c}O(1) \\
O\left(R^{T+1}\right) \\
\end{array}$ & $\checkmark$ & $x$ & $\checkmark$ & $\boldsymbol{J}^{2}$ \\
\hline scrypt & $\begin{array}{c}O(1) \\
O\left(R^{2}\right) \\
\end{array}$ & $\checkmark$ & $x$ & $!$ & $x$ \\
\hline
\end{tabular}

$\boldsymbol{J}$ - Has protection; $\boldsymbol{X}$ - Has no protection; ! - Partial protection; - - Nothing declared.

Table 2: Security overview of the PHSs considered the state of the art.

ted from the reference guides, manuals and articles describing and/or analyzing these solutions, also including third-party analysis. Hence, we refer the interested reader to the original sources for details on each of these algorithms: Argon2 (BIRYUKOV; DINU; KHOVRATOVICH, 2016), battcrypt (THOMAS, 2014), Catena(FORLER; LUCKS; WENZEL, 2013), Lyra (ALMEIDA et al., 2014), Pomelo (WU, 2015), yescrypt (PESLYAK, 2015), scrypt (PERCIVAL, 2009), and garbage collector attacks (FORLER et al., 2014).

\footnotetext{
${ }^{2}$ Provides protection when in its "read-write" mode (YESCRYPT_RW) (FORLER et al., 2014).
} 


\section{PERFORMANCE FOR DIFFERENT SETTINGS}

In our assessment of Lyra2's performance, we used an SSE-enabled implementation of Blake2b's compression function (AUMASSON et al., 2013) as the underlying sponge's $f$ function of Algorithm 5. According to our tests, using SSE (Streaming SIMD Extensions, where SIMD stands for Single Instruction, Multiple Data) instructions allow performance gains of $20 \%$ to $30 \%$ in comparison with non-SSE settings, we therefore only consider such optimized implementations in this document.

One important note about this implementation is that, as discussed in Section 4.4, the least significant 512 bits of the sponge's state are set to zeros, while the remainder 512 bits are set to Blake2b's Initialization Vector. Also, to prevent the IV from being overwritten by user-defined data, the sponge's capacity $c$ employed when absorbing the user's input (line 4 of Algorithm 5) is kept at 512 bits, but reduced to 256 bits in the remainder of the algorithm to allow a higher bitrate (namely, of 768 bits) during most of its execution. The implementations employed, as well as test vectors, are available at $<$ www.lyra2.net $>$.

\subsection{Benchmarks for Lyra2}

The results obtained with a SSE-optimized single-core implementation of Lyra2 are illustrated in Figure 14. The results depicted correspond to the average 
execution time of Lyra2 configured with $C=256, \rho=1, b=768$ bits (i.e., the inner state has 256 bits), and different $T$ and $R$ settings, giving an overall idea of possible combinations of parameters and the corresponding usage of resources.

As shown in this figure, Lyra2 is able to execute in: less than $1 \mathrm{~s}$ while using up to $400 \mathrm{MB}$ (with $R=2^{14}$ and $T=5$ ) or up to $1 \mathrm{~GB}$ of memory (with $R \approx 4.2 \cdot 10^{4}$ and $T=1$ ); or in less than $5 \mathrm{~s}$ with $1.6 \mathrm{~GB}$ (with $R=2^{16}$ and $T=6)$. All tests were performed on an Intel Xeon E5-2430 (2.20 GHz with 12 Cores, 64 bits) equipped with 48 GB of DRAM, running Ubuntu 14.04 LTS 64 bits. The source code was compiled using gcc 4.9.2.

The same Figure 14 also compares Lyra2 with the scrypt "SSE-enabled" implementation publicly available at $<$ www.tarsnap.com/scrypt.html $>$, using the parameters suggested by scrypt's author in (PERCIVAL, 2009), namely, $b=8192$ and $p=1$. The results obtained show that, to achieve a memory usage and processing time similar to that of scrypt, Lyra2 could be configured with $T \approx 6$.

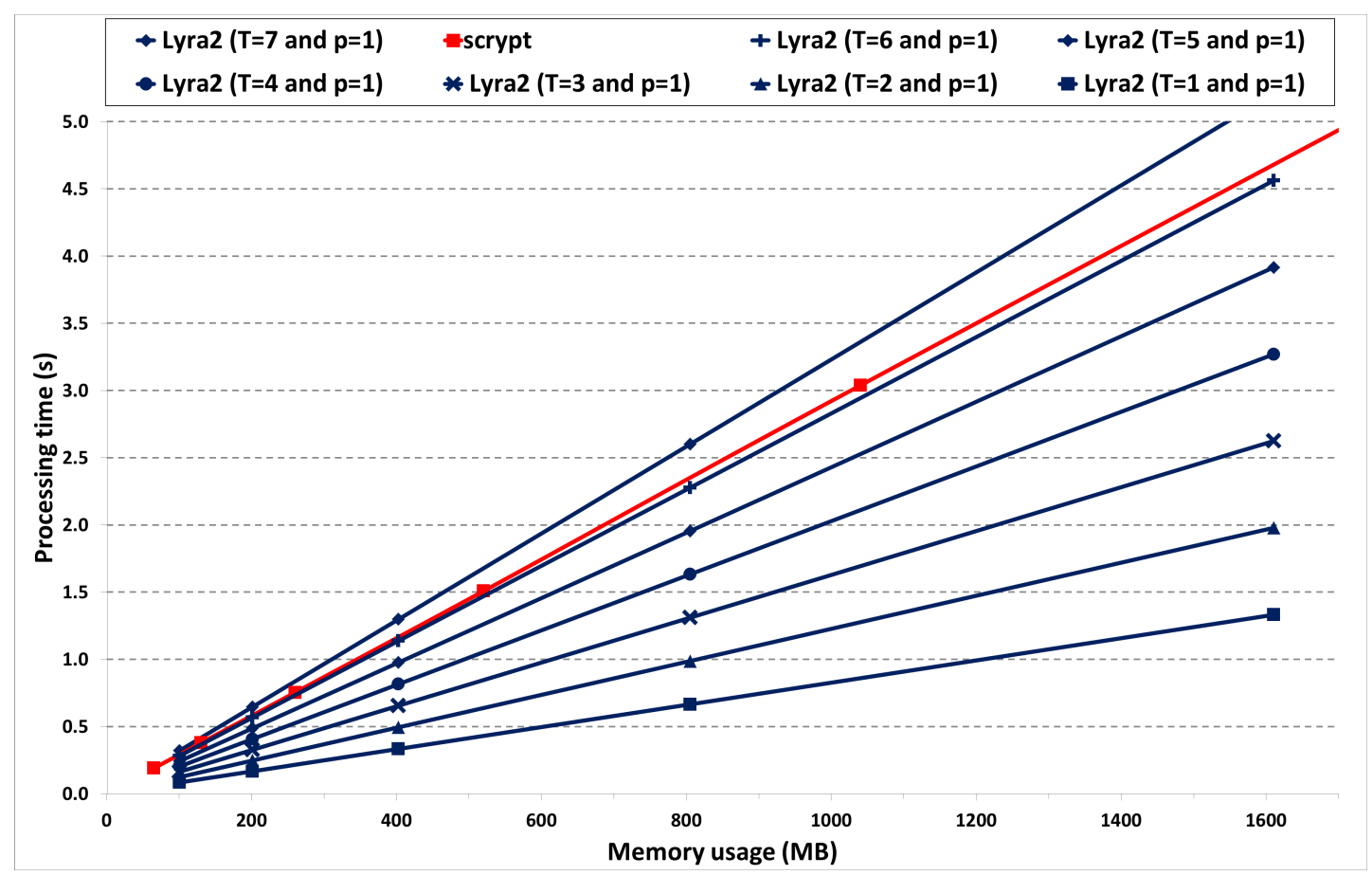

Figure 14: Performance of SSE-enabled Lyra2, for $C=256, \rho=1, p=1$, and different $T$ and $R$ settings, compared with SSE-enabled scrypt. 
We also performed tests aiming to compare the performance of Lyra2 and the other 5 memory-hard PHC finalists: Argon2, battcrypt, Catena, POMELO, and yescrypt. Parameterizing each algorithm to ensure a fair comparison between them is not an obvious task, however, because the amount of resources taken by each PHS in a legitimate platform is a user-defined parameter chosen to influence the cost of brute-force guesses. Hence, ideally one would have to find the parameters for each algorithm that normalizes the costs for attackers, for example in terms of energy and chip area in hardware, the cost of memory-processing tradeoffs in software, or the throughput in highly parallel platforms such as GPUs.

In the absence of a complete set of optimized implementations for gathering such data, a reasonable approach is to consider the minimum parameters suggested by the authors of each scheme: even though this analysis does not ensure that the attack costs are similar to all schemes, it at least shows what the designers recommend as the bare minimum cost for legitimate users. The results, which basically confirm existing analysis performed in (BROZ, 2014), are depicted in Figure 15, which shows that Lyra2 is a very competitive solution in terms of

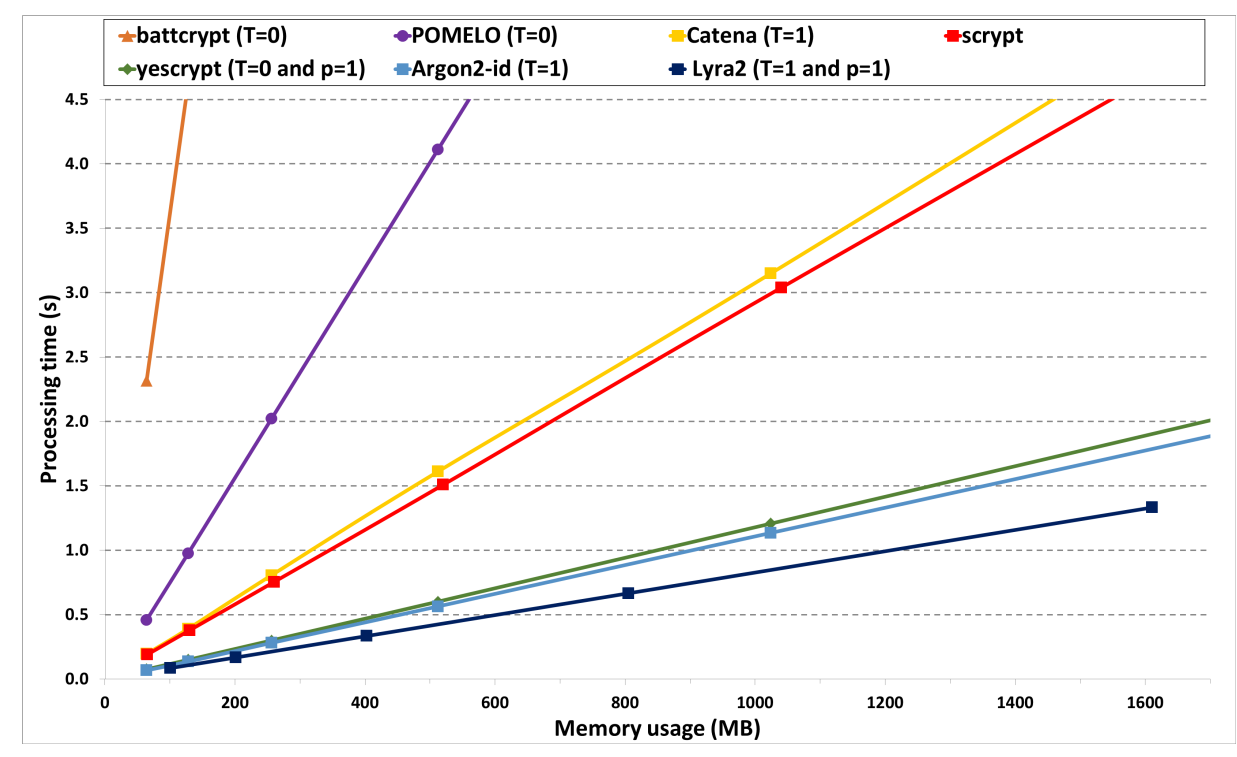

Figure 15: Performance of SSE-enabled Lyra2, for $C=256, \rho=1, p=1$, and different $T$ and $R$ settings, compared with SSE-enabled scrypt and memory-hard PHC finalists with minimum parameters. 
performance.

Another normalization can be made if we consider that, in a nutshell, a memory-hard PHS consists of an iterative program that initializes and revisits several memory positions. Therefore, one can assess each algorithm's performance when they are all parameterized to make the same number of calls to the underlying non-invertible (possible cryptographic) function. The goal with this normalization is to evaluate how efficiently the underlying primitive is employed by the scheme, giving an overall idea of its throughput. It also provides some insight on how much that primitive should be optimized to obtain similar processing times for a given memory usage, or even if it is worth replacing that primitive by a faster algorithm (assuming that the scheme is flexible enough to allow users to do so).

The benchmark results are shown in Figure 16, in which lines marked with the same symbol (e.g., $\mathbf{\square}$ or $\bullet$ ) denote algorithms configured with a similar number

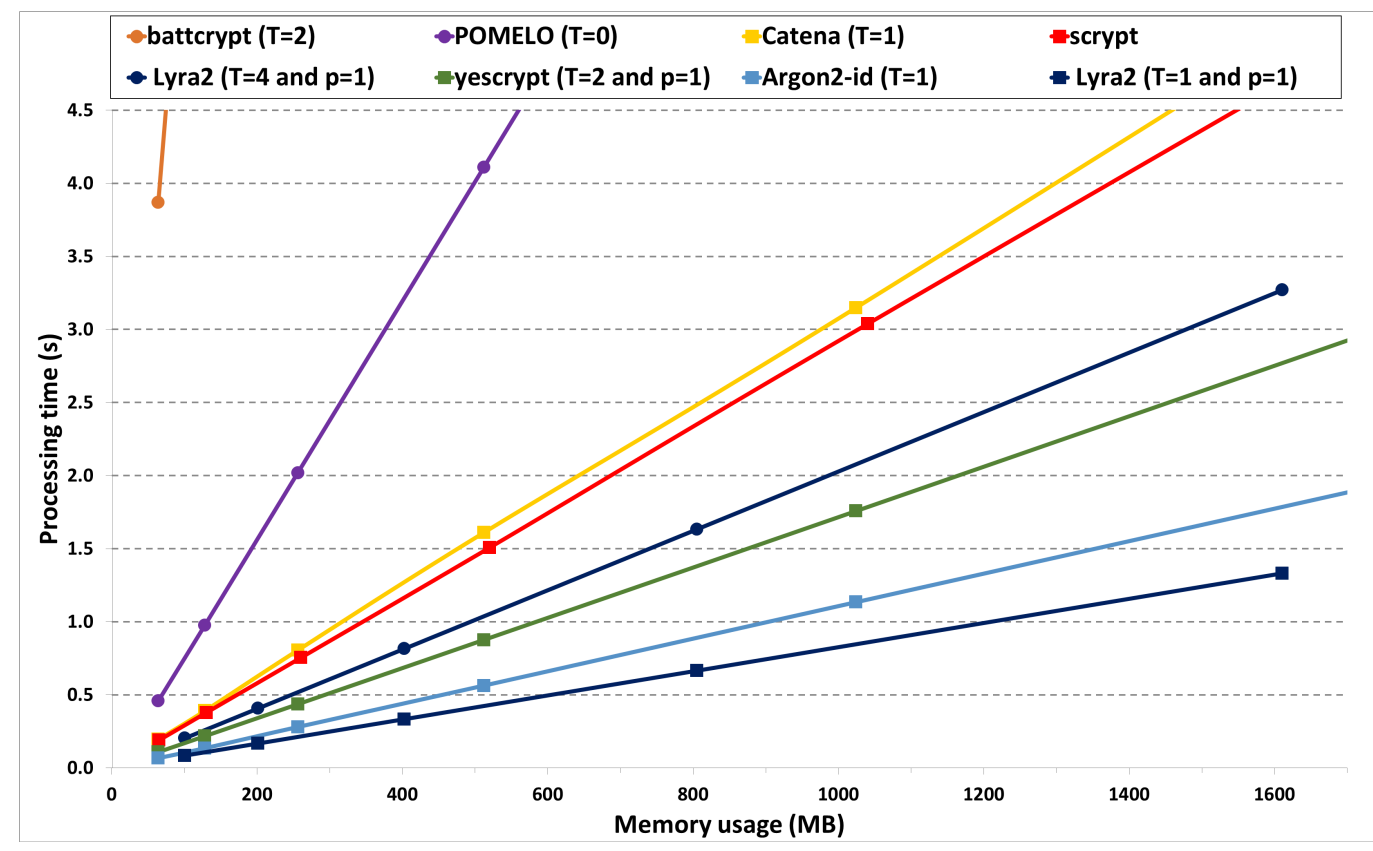

Figure 16: Performance of SSE-enabled Lyra2, for $C=256, \rho=1, p=1$ and different $T$ and $R$ settings, compared with SSE-enabled scrypt and memoryhard PHC finalists with a similar number of calls to the underlying function (comparable configurations are marked with the same symbol, $\mathbf{\square}$ or $\bullet$ ). 


\begin{tabular}{c|c|c}
\hline Algorithm & Calls to underlying primitive & SIMD instructions \\
\hline Argon2 & $2 \cdot T \cdot M$ & Yes \\
battcrypt & $\left\{2^{\lfloor T / 2\rfloor} \cdot[(T \bmod 2)+2]+1\right\} \cdot M$ & No \\
Catena $^{1}$ & $(T+1) \cdot M$ & Yes \\
Lyra2 & $(T+1) \cdot M$ & Yes \\
POMELO & $\left(3+2^{2 T}\right) \cdot M$ & No \\
yescrypt & $T \cdot M$ & Yes \\
\hline
\end{tabular}

Table 3: PHC finalists: calls to underlying primitive in terms of their time and memory parameters, $T$ and $M$, and their implementations.

of calls to the underlying function. The exact choice of parameters in this figure comes from Table 3, which shows how each memory-hard PHC finalist handles the time- and memory-cost parameters (respectively, $T$ and $M$ ), based on the analysis of the documentation provided by their authors (PHC, 2013; PESLYAK, 2015; PHC wiki, 2014). The source codes were all compiled with the -03 option whenever the authors did not specify the use of another compilation flag. Once again, Lyra2 displays a superior performance, which is a direct result of adopting an efficient and reduced-round cryptographic sponge as underlying primitive.

One remark concerning these results is that, as also shown in Table 3, the implementations of battcrypt and POMELO employed in the benchmarks do not employ SIMD instructions, which means that the comparison is not completely fair. Nevertheless, even if such advanced instructions are able to reduce their processing times by half, their relative positions in the figure would not change.

In addition of these results, a third-party implementation (GONÇALVES, 2014) has shown that it is possible to reduce Lyra2's processing time even more in modern processors equipped with AVX2 (Advanced Vector Extensions 2) instructions. With that study, the authors produced a piece of code that runs $30 \%$ faster than our SSE-optimized implementation (GONÇALVES; ARANHA, 2005).

\footnotetext{
${ }^{1}$ The exact number of calls to the underlying cryptographic primitive in Catena is given by equation $\left(g-g_{0}+1\right) \cdot(T+1) \cdot M$, where $g$ and $g_{0}$ are, respectively, the current and minimum garlic. However, since normally $g=g_{0}$, here we use the simplified equation $(T+1) \cdot M$.
} 


\subsection{Benchmarks for BlaMka.}

In terms of performance, our analysis indicates that the throughput of BlaMka when compared with Blake2b drops less in CPUs than in dedicated hardware, meaning that it is more advantageous for regular users than it is for attackers. This analysis is based on benchmarks of Blake2b's $G$ and BlaMka's $G_{\mathrm{t} 1 \mathrm{~s}}$ functions performed in CPUs, comparing the results with hardware implementations made by Jonatas F. Rossetti under the supervision of Prof. Wilson V. Ruggiero.

For all implementations, we measured the average throughput of one round of $G$ and $G_{\text {tls }}$ (i.e., for 256 bits of input/output), which does not correspond to the entire Blake2b or BlaMka algorithms but should be enough for a comparative analysis between these permutation functions. In addition, for simplicity, along the discussion we ignore ancillary (e.g, memory-related) operations that should appear in any algorithm using either Blake2b or BlaMka as underlying hash function. As later discussed in Section 6.3, this simplification leads to numbers implying an impact much more significant than those actually observed in algorithms involving a large amount of memory-related operations, such as Lyra2 itself. Nevertheless, the resulting analysis is useful to evaluate how the adoption of BlaMka or Blake2b as underlying hash function impacts the "processing" portion of an algorithm's total execution time.

Table 4 shows the benchmark results obtained with software implementations of $G$ and $G_{\mathrm{tls}}$. All tests were performed on an Intel Xeon E5-2430 $(2.20 \mathrm{GHz}$ with 12 Cores, 64 bits) equipped with 48 GB of DRAM and running Ubuntu 14.04 LTS 64 bits. The source code was compiled using gcc 4.9.3 with -03 optimization, and SIMD (Stands for Single Instruction, Multiple Data) instructions were not used, in order to implement these functions in the most generic way possible. 


\begin{tabular}{c|c|c|c|c|c|c|c|c}
\hline Microarchitecture & $\begin{array}{c}\text { CPU } \\
\text { Family }\end{array}$ & Model & $G$ & Implem. & $\begin{array}{c}\text { T. Power } \\
(\mathrm{W})\end{array}$ & $\begin{array}{c}\text { Max. Frequency } \\
(\mathrm{MHz})\end{array}$ & $\begin{array}{c}\text { Throughput } \\
(\text { Gbps })\end{array}$ & $\begin{array}{c}\text { Throughput } \\
\text { Ratio }\end{array}$ \\
\hline Sandy Bridge & $\begin{array}{c}\text { Intel } \\
\text { Xeon }\end{array}$ & E5-2430 & $\begin{array}{c}\text { Blake2b } \\
\text { BlaMka }\end{array}$ & $\begin{array}{c}\text { Generic } \\
\text { Generic }\end{array}$ & 95 & $2200^{2}$ & 51.578 & 1 \\
\hline
\end{tabular}

Table 4: Data related of the tests performed in CPU, executing just one round of $G$ function (i.e., 256 bits of output).

As shown in Table 4's rightmost column, BlaMka's $G_{\mathrm{tls}}$ function has approximately $42 \%$ of the throughput capacity of the original Blake2b's $G$ permutation function in CPUs. Hence, ignoring ancillary operations, any algorithm using BlaMka as underlying hash function should observe a similar impact on its throughput, whether it is a legitimate or an attacker's implementation.

The hardware descriptions for $G$ and $G_{\mathrm{tls}}$, in turn, were made directly from definition of the algorithms described in Figure 4, using basic combinational and sequential components, such as registers, multiplexers, adders, multipliers, and 64-bit XORs. The rotation and shift left operations present on BlaMka were implemented as simple changes in wire connections, as it is known in advance how many bits should be rotated and left shifted. Two versions were developed for the purposed of benchmarking. In the first, simply denoted Generic", no special adder or multiplier were used, leaving for the implementation tools the task of choosing some special algorithm. In the second, denoted "Opt" to indicate further optimizations, the hardware descriptions were made using specific combinational multipliers — namely, Dadda Multipliers (DADDA, 1965; DADDA, 1976) — and Carry Save Adders, using registers along the critical path to reduce latency.

Both Generic and Opt descriptions were implemented in FPGA using Xilinx ISE Design Suite (XILINX, 2013) and Xilinx Vivado Design Suite (XILINX, 2016) for multiple FPGA families of different fabrication technologies (Tables 5 and 6). For the implementations on ASICs, the Synopsys Design Compiler tool

\footnotetext{
${ }^{2}$ During the tests the TurboBoost was turned off.
} 
(SYNOPSYS, 2010) was used in a 90nm cell library (Table 6). These tools were used with default implementation parameters, aiming to achieve the most generic results possible.

Tables 5 and 6 show the results obtained for FPGA and ASICs implementations, respectively. In those tables, one can see that the throughput ratio of BlaMka instead of Blake2b is in most cases worse than those one obtained with CPUs. Namely, in the FPGA-based implementations, BlaMka's throughput from $29.5 \%$ to $58.9 \%$ of Blake2b's throughput instead of $42 \%$ observed in software, but in 9 out of 12 them BlaMka's multiplication-hardened permutation was higher on hardware than on software. The ASICs' implementations, on the other hand, could cope considerably better with the $G_{\mathrm{t} 1 \mathrm{~s}}$ structure, so BlaMka's throughput became $45.3 \%$ to $89.5 \%$ of Blake2b's in this platform.

Nevertheless, in practice, we need to take into account of the area employed by the implementations, since, from an attackers' perspective, doubling the area of one implementation only makes sense if the resulting throughput more than doubles; otherwise, it would be more beneficial to have two instances of the algorithm running in parallel testing different passwords, as this would result in twice as much area with twice the throughput. The rightmost column of Tables 5 and 6, the "Relative Throughput", take this observation into account, as they indicate how much throughput is lost with the adoption of BlaMka instead of Blake2b assuming the same amount of silicon area is available for implementing both algorithms. Specifically, BlaMka's relative throughput ranges from $4 \%$ to $38.3 \%$ of the throughput observed with Blake2b, remaining, thus, below the $42 \%$ obtained in software. Therefore, according to this analysis, the adoption of BlaMka as underlying sponge by legitimate users with software-based implementations of the algorithm does improve defense against attackers with access to dedicated hardware. 
FPGA (ISE Design Suite)

\begin{tabular}{|c|c|c|c|c|c|c|c|c|c|c|}
\hline \multirow{2}{*}{ Technology } & \multirow{2}{*}{$\begin{array}{l}\text { FPGA } \\
\text { Family }\end{array}$} & \multirow{2}{*}{ G } & \multirow{2}{*}{ Implem. } & \multicolumn{2}{|c|}{ Area } & \multirow{2}{*}{$\begin{array}{c}\text { T. Power } \\
(\mathrm{mW})\end{array}$} & \multirow{2}{*}{$\begin{array}{l}\text { Max. Frequency } \\
(\mathrm{MHz})\end{array}$} & \multicolumn{2}{|c|}{ Throughput } & \multirow{2}{*}{$\begin{array}{c}\text { Relative } \\
\text { Throughput }\end{array}$} \\
\hline & & & & Slices & Ratio & & & Gbps & Ratio & \\
\hline \multirow{3}{*}{$65 \mathrm{~nm}$} & \multirow{3}{*}{ Virtex 5} & Blake2b & Generic & 237 & 1 & 1529 & 257.003 & 4.112 & 1 & 1 \\
\hline & & BlaMka & Generic & 383 & 1.616 & 1280 & 75.689 & 1.211 & 0.295 & 0.182 \\
\hline & & BlaMka & Opt & 2030 & 8.565 & 1511 & 88.511 & 1.416 & 0.344 & 0.040 \\
\hline \multirow{3}{*}{$28 \mathrm{~nm}$} & \multirow{3}{*}{ Kintex 7} & Blake2b & Generic & 359 & 1 & 372 & 364.299 & 5.829 & 1 & 1 \\
\hline & & BlaMka & Generic & 433 & 1.206 & 232 & 109.397 & 1.750 & 0.300 & 0.249 \\
\hline & & BlaMka & Opt & 2306 & 6.423 & 463 & 142.450 & 2.279 & 0.391 & 0.061 \\
\hline \multirow{3}{*}{$45 \mathrm{~nm}$} & \multirow{3}{*}{ Spartan 6} & Blake2b & Generic & 239 & 1 & 45 & 141.663 & 2.267 & 1 & 1 \\
\hline & & BlaMka & Generic & 206 & 0.862 & 94 & 46.757 & 0.748 & 0.330 & 0.383 \\
\hline & & BlaMka & Opt & 1592 & 6.661 & 327 & 104.373 & 1.336 & 0.589 & 0.088 \\
\hline
\end{tabular}

FPGA (Vivado Design Suite)

\begin{tabular}{|c|c|c|c|c|c|c|c|c|c|c|}
\hline \multirow{2}{*}{ Technology } & \multirow{2}{*}{$\begin{array}{l}\text { FPGA } \\
\text { Family }\end{array}$} & \multirow{2}{*}{$G$} & \multirow{2}{*}{ Implem. } & \multicolumn{2}{|c|}{ Area } & \multirow{2}{*}{$\begin{array}{c}\text { T. Power } \\
(\mathrm{mW})\end{array}$} & \multirow{2}{*}{$\begin{array}{c}\text { Max. Frequency } \\
(\mathrm{MHz})\end{array}$} & \multicolumn{2}{|c|}{ Throughput } & \multirow{2}{*}{$\begin{array}{c}\text { Relative } \\
\text { Throughput }\end{array}$} \\
\hline & & & & Slices & Ratio & & & Gbps & Ratio & \\
\hline \multirow{3}{*}{$16 \mathrm{~nm}$} & Kintex & Blake2b & Generic & 144 & 1 & 690 & 500.250 & 8.004 & 1 & 1 \\
\hline & Ultra & BlaMka & Generic & 210 & 1.458 & 692 & 167.842 & 2.685 & 0.335 & 0.230 \\
\hline & SCALE+ & BlaMka & Opt & 1216 & 8.444 & 766 & 194.818 & 3.117 & 0.389 & 0.046 \\
\hline \multirow{3}{*}{$20 \mathrm{~nm}$} & Kintex & Blake2b & Generic & 148 & 1 & 958 & 280.741 & 4.492 & 1 & 1 \\
\hline & Ultra & BlaMka & Generic & 218 & 1.473 & 956 & 99.433 & 1.591 & 0.354 & 0.240 \\
\hline & SCALE & BlaMka & Opt & 1184 & 8.000 & 1024 & 135.538 & 2.169 & 0.483 & 0.060 \\
\hline \multirow{3}{*}{$28 \mathrm{~nm}$} & \multirow{3}{*}{ Virtex 7} & Blake2b & Generic & 139 & 1 & 273 & 219.443 & 3.511 & 1 & 1 \\
\hline & & BlaMka & Generic & 188 & 1.353 & 276 & 100.291 & 1.605 & 0.457 & 0.338 \\
\hline & & BlaMka & Opt & 994 & 7.151 & 317 & 84.545 & 1.353 & 0.385 & 0.054 \\
\hline
\end{tabular}

Table 5: Data related of the initial tests performed in FPGA, executing just one round of $G$ function (i.e., 256 bits of output).

FPGA (ISE Design Suite)

\begin{tabular}{|c|c|c|c|c|c|c|c|c|c|c|}
\hline Technology & $\begin{array}{l}\text { FPGA } \\
\text { Family }\end{array}$ & $G$ & Implem. & \multicolumn{2}{|c|}{ Area } & $\begin{array}{c}\text { T. Power } \\
(\mathrm{mW})\end{array}$ & $\begin{array}{c}\text { Max. Frequency } \\
(\mathrm{MHz})\end{array}$ & \multicolumn{2}{|c|}{ Throughput } & $\begin{array}{c}\text { Relative } \\
\text { Throughput }\end{array}$ \\
\hline \multirow{2}{*}{$90 \mathrm{~nm}$} & \multirow{2}{*}{ Spartan $3 \mathrm{E}$} & Blake2b & Generic & 508 & 1 & 162 & 98.097 & 1.570 & 1 & 1 \\
\hline & & BlaMka & Opt & 3567 & 7.022 & 160 & 57.894 & 0.741 & 0.472 & 0.067 \\
\hline
\end{tabular}

ASIC (Synopsys Design Compiler)

\begin{tabular}{|c|c|c|c|c|c|c|c|c|c|c|}
\hline \multirow{2}{*}{ Technology } & \multirow{2}{*}{ Family } & \multirow{2}{*}{$G$} & \multirow{2}{*}{ Implem. } & \multicolumn{2}{|c|}{ Area } & \multirow{2}{*}{$\begin{array}{l}\text { T. Power } \\
(\mu \mathrm{W})\end{array}$} & \multirow{2}{*}{$\begin{array}{c}\text { Max. Frequency } \\
(\mathrm{MHz})\end{array}$} & \multicolumn{2}{|c|}{ Throughput } & \multirow{2}{*}{$\begin{array}{c}\text { Relative } \\
\text { Throughput }\end{array}$} \\
\hline & & & & $\mu \mathrm{m}^{2}$ & Ratio & & & Gbps & Ratio & \\
\hline \multirow{2}{*}{$90 \mathrm{~nm}$} & SAED & Blake2b & Generic & 40191 & 1 & 222 & 239.808 & 3.837 & 1 & 1 \\
\hline & $90 \mathrm{~nm}$ & BlaMka & Opt & 142911 & 3.556 & 637 & 245.700 & 3.145 & 0.820 & 0.231 \\
\hline
\end{tabular}

Table 6: Data related of the initial tests performed in dedicated hardware (that present advantage against $\mathrm{CPU}$ ), executing just one round of $G$ function (i.e., 256 bits of output). 


\subsection{Benchmarks for Lyra2 with BlaMka}

Since BlaMka includes a larger number of operations than Blake2b, it is natural that the performance of Lyra2 when it employs BlaMka instead of Blake2b as underlying permutation will be lower than reported in Section 6.1; nevertheless, the impact is unlikely to be as high as observed in Section 6.2 , since memoryrelated operations play an important role in the algorithm's total execution time. To assess the impacts of BlaMka over Lyra2's efficiency, we conducted some benchmarks as described as follows.

Figure 17 shows the results for Lyra2 configured with $p=1$, comparing it with the other memory-hard PHC finalists. As observed in this figure, Lyra2's performance remains quite competitive: for a given memory usage, Lyra2 is slower than yescrypt and Argon2 configured with minimal settings, but remains faster than yescrypt when both are configured to make the same number of calls to the underlying function (i.e., for yescrypt with $T=2$ and Lyra2 with $T=1$ ).

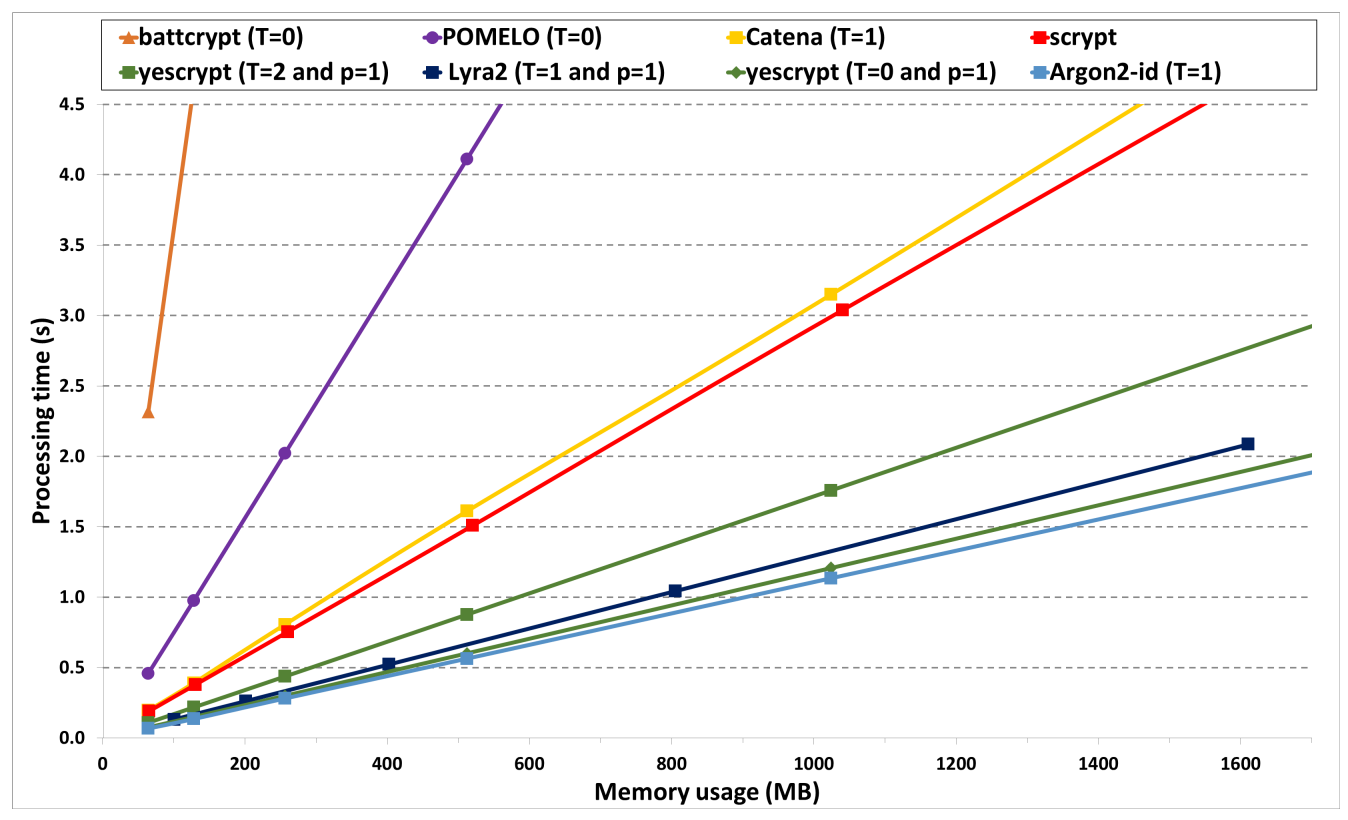

Figure 17: Performance of SSE-enabled Lyra2 with BlaMka $G$ function, for $C=$ 256, $\rho=1, p=1$, and different $T$ and $R$ settings, compared with SSE-enabled scrypt and memory-hard PHC finalists (configurations with a similar number of calls to the underlying function are marked with the same symbol, $\mathbf{\square})$. 


\subsection{Expected attack costs}

Considering that the cost of DDR3 SO-DIMM memory chips is currently around U\$3.1/GB (TRENDFORCE, 2016), Table 7 shows the cost added by Lyra2 with $T=1$ and $T=5$ when an attacker tries to crack a password in 1 year using the above reference hardware, for different password strengths — we refer the reader to (NIST, 2011, Appendix A) for a discussion on how to compute the approximate entropy of passwords.

These costs are obtained considering the total number of instances that need to run in parallel to test the whole password space in 365 days and supposing that testing a password takes the same amount of time as in our testbed. Notice that, in a real scenario, attackers would also have to consider costs related to wiring and energy consumption of memory chips, besides the cost of the processing cores themselves.

We notice that if the attacker uses a faster platform (e.g., an FPGA or a more powerful computer), these costs should drop proportionally, since a smaller number of instances (and, thus, memory chips) would be required for this task. Similarly, if the attacker employs memory devices faster than regular DRAM (e.g., SRAM or registers), the processing time is also likely to drop, reducing the number of instances required to run in parallel.

Nonetheless, in this case the resulting memory-related costs may actually be significantly bigger due to the higher cost per GB of such memory devices. Anyhow, the numbers provided in Table 7 are not intended as absolute values, but rather a reference on how much extra protection one could expect from using Lyra2, since this additional memory-related cost is the main advantage of any PHS that explores memory usage when compared with those that do not. 


\begin{tabular}{c|c|c|c|c|c|c|c|c}
\hline \multirow{2}{*}{$\begin{array}{c}\text { Password } \\
\text { entropy (bits) }\end{array}$} & \multicolumn{4}{|c|}{ Memory usage (MB) for $T=1$} & \multicolumn{4}{c}{ Memory usage (MB) for $T=5$} \\
\cline { 2 - 9 } & 200 & 400 & 800 & 1,600 & 200 & 400 & 800 & 1,600 \\
\hline 35 & 114.5 & 457.0 & $1.8 \mathrm{k}$ & $7.3 \mathrm{k}$ & 333.5 & $1.3 \mathrm{k}$ & $5.6 \mathrm{k}$ & $21.5 \mathrm{k}$ \\
40 & $3.7 \mathrm{k}$ & $14.6 \mathrm{k}$ & $58.4 \mathrm{k}$ & $233.6 \mathrm{k}$ & $10.7 \mathrm{k}$ & $42.8 \mathrm{k}$ & $171.5 \mathrm{k}$ & $687.1 \mathrm{M}$ \\
45 & $117.3 \mathrm{k}$ & $468.0 \mathrm{k}$ & $1.9 \mathrm{M}$ & $7.5 \mathrm{M}$ & $341.5 \mathrm{k}$ & $1.4 \mathrm{M}$ & $5.5 \mathrm{M}$ & $22.0 \mathrm{M}$ \\
50 & $3.8 \mathrm{M}$ & $15.0 \mathrm{M}$ & $59.8 \mathrm{M}$ & $239.2 \mathrm{M}$ & $10.9 \mathrm{M}$ & $43.8 \mathrm{M}$ & $175.6 \mathrm{M}$ & $703.6 \mathrm{M}$ \\
55 & $120.1 \mathrm{M}$ & $479.2 \mathrm{M}$ & $1.9 \mathrm{~B}$ & $7.7 \mathrm{~B}$ & $349.7 \mathrm{M}$ & $1.4 \mathrm{~B}$ & $5.6 \mathrm{~B}$ & $22.5 \mathrm{~B}$ \\
\hline
\end{tabular}

Where: $\mathrm{k}($ kilo $)=\times 1,000 ; \quad \mathrm{M}($ Million $)=\times 1,000,000 ; \quad \mathrm{B}($ Billion $)=\times 1,000,000,000$.

Table 7: Memory-related cost (in U\$) added by the SSE-enable version of Lyra2 with $T=1$ and $T=5$, for attackers trying to break passwords in a 1-year period using an Intel Xeon E5-2430 or equivalent processor.

Finally, when compared with existing solutions that do explore memory usage, Lyra2 is advantageous due to the elevated processing costs of attack venues involving time-memory trade-offs, effectively discouraging such approaches.

Indeed, from Equation 5.8 and for $T=5$, the processing cost of an attack against Lyra2 using half of the memory defined by the legitimate user would be $O\left((3 / 2)^{2 T} R^{2}\right)$, which translates to $(3 / 2)^{2 \cdot 5} \cdot\left(2^{14}\right)^{2} \approx 2^{34} \sigma$ if the algorithm operates regularly with $400 \mathrm{MB}$, or $(3 / 2)^{2 \cdot 5} \cdot\left(2^{16}\right)^{2} \approx 2^{38} \sigma$ for a memory usage of 1.6 GB. For the same memory usage settings, the total cost of a memory-free attack against scrypt would be approximately $\left(2^{15}\right)^{2} / 2=2^{29}$ and $\left(2^{17}\right)^{2} / 2=2^{33}$ calls to BlockMix, whose processing time is approximately $2 \sigma$ for the parameters employed in our experiments. As expected, such elevated processing costs resulting from this small memory usage reduction are prone to discourage attack venues that try to avoid the memory costs of Lyra2 by means of extra processing.

\subsection{Summary}

This chapter discussed the performance of Lyra2, showing that it is quite competitive with other PHS solutions considered among the state-of-the-art, even surpassing some of them, allowing legitimate users to use a large amount of memory while keeping the algorithm's execution time within reasonable levels. 
This positive result is, at least in part, due to the adoption of a reducedround cryptographic sponge as underlying function, which allows more memory positions to be covered in a same amount of time than what would be possible with the application of a full-round sponge. The use of a higher bitrate during the most of the algorithm's execution (768 bits in our tests) also contributes to this higher speed without decreasing its security against possible cryptanalytic attacks to a low level, as even so the sponge's capacity can be kept quite high (256 bits in our tests).

The choice of software-oriented cryptographic sponges is similarly important, as it not only leads to high performance, but also gives more advantage to legitimate users than to attackers.

Finally, Lyra2's memory matrix was designed to allows legitimate users to take advantage of memory hierarchy features, such as caching and prefetching, which optimizes the portion of the execution time related to memory operations even with common hardware available in software-oriented platforms. These properties can also be combined with optimization strategies from modern processors, such as vectorized instructions (e.g., SSE and AVX). The end result is a fast and flexible design, which leads to a considerable cost of password-cracking hardware, comparable (and not uncommonly superior) to the best results available in the current state-of-the-art. 


\section{FINAL CONSIDERATIONS}

In this document, we presented Lyra2, a password hashing scheme (PHS) that allows legitimate users to fine tune memory and processing costs according to the desired level of security and resources available in the target platform. For achieving this goal, Lyra2 builds on the properties of sponge functions operating in a stateful mode, creating a strictly sequential process. Indeed, the whole memory matrix of the algorithm can be seen as a huge state, which changes together with the sponge's internal state.

The ability to control Lyra2's memory usage allows legitimate users to thwart attacks using parallel platforms. This can be accomplished by raising the total memory required by the several cores beyond the amount available in the attacker's device. In summary, the combination of a strictly sequential design, the high costs of exploring time-memory trade-offs, and the ability to raise the memory usage beyond what is attainable with similar-purpose solutions (e.g., scrypt) for a similar security level and processing time make Lyra2 an appealing PHS solution.

\subsection{Publications and other results}

The following adoptions, publications, presentations, submissions and envisioned papers are a direct or indirect result of the research effort carried out during the elaboration of this thesis: 
- PHC special recognition: Lyra2 received a special recognition for its elegant sponge-based design, and alternative approach to side-channel resistance combined with resistance to slow-memory attacks (PHC, 2015b).

- BlaMka's adoption: the winner of the PHC, Argon2, adopts BlaMka by default as its permutation function (BIRYUKOV; DINU; KHOVRATOVICH, 2016).

- Lyra2's adoptions: the Vertcoin electronic currency announced that it is migrating from scrypt to Lyra2 due to the excellent performance against custom mining hardware, as well as to the latter's ability to fine tune memory usage and processing time Lyra2 (A432511, 2014; DAY, 2014). Furthermore, a few months ago one of the major bitcoin mining software on GPU, the Sgminer, added support to Lyra2 in its distribution package (CRYPTO MINING, 2015).

- Journal Articles: in (ALMEIDA et al., 2014), we present the Lyra algorithm, describing the preliminary ideas that gave rise to Lyra2; in (ANDRADE et al., 2016), we describe Lyra2 and provide a brief security and performance analysis of the algorithm.

- Extended abstract: in (ANDRADE; SIMPLICIO JR, 2014b), we presented the initial ideas and results of Lyra2, and took the opportunity of the event to discuss these results with other graduate students from the graduate program of Electrical Engineering (Computer Engineering area) at Escola Politecnica; in (ANDRADE; SIMPLICIO JR, 2014a), we gave an oral presentation at LatinCrypt'14 with some preliminary results; and in (ANDRADE; SIMPLICIO JR, 2016) we present the current status of our project.

- Award: recently, we received a best PhD project award due to Lyra2 design 
and analysis (ANDRADE; SIMPLICIO JR, 2016; ICISSP, 2016).

\subsection{Future works}

As future work, we plan to provide a more detailed performance and security analysis of Lyra2 with different underlying sponges and in different platforms. Among those sponges, in especial we intend to evaluate multiplication-hardened solutions, including BlaMka, since this kind of solutions would increase the protections against attacks performed with dedicated hardware.

Another interesting subject of research involves adapting Lyra2's design to allow parallel tasks during the password hashing process. This approach would allow legitimate users to take advantage of multi-core architectures (either in CPU or in GPU) for accelerating the password hashing process in their own platforms and, hence, potentially raise the memory usage for some target processing time.

Finally, we can also evaluate the consequences to adopt a password independent approach on the Lyra2 structure, aiming make it totally resistant against cache-timing attacks, evaluating the impacts of such approach when experimentally performing a slow-memory attack. 


\section{REFERENCES}

A432511. PoW Algorithm Upgrade: Lyra2 - Vertcoin. 2014. < https: //vertcoin.org/pow-algorithm-upgrade-lyra2/>. Accessed: 2015-05-06.

ALMEIDA, L. C.; ANDRADE, E. R.; BARRETO, P. S. L. M.; SIMPLICIO JR, M. A. Lyra: Password-Based Key Derivation with Tunable Memory and Processing Costs. Journal of Cryptographic Engineering, Springer Berlin Heidelberg, v. 4, n. 2, p. 75-89, 2014. ISSN 2190-8508. See also $<$ http://eprint.iacr.org/2014/030>.

ANDRADE, E. R.; SIMPLICIO JR, M. A. Lyra2: a password hashing schemes with tunable memory and processing costs. 2014. Third International Conference on Cryptology and Information Security in Latin America, LATINCRYPT'14. Florianópolis, Brazil.<http://latincrypt2014.labsec.ufsc.br/>. Accessed: 2015-05-06.

Lyra2: Um Esquema de Hash de Senhas com custos de memória e processamento ajustáveis. São Paulo, SP, Brazil: III WPG-EC. < http: //www2.pcs.usp.br/wpgec/2014/index.htm>. Accessed: 2016-04-20., 2014. 53-55 p.

. Lyra2: Efficient Password Hashing with high security against TimeMemory Trade-Offs. In: INSTITUTE FOR SYSTEMS AND TECHNOLOGIES OF INFORMATION. Doctoral Consortium - Proceedings of 2nd International Conference on Information Systems Security and Privacy, ICISSP 2016. Rome, Italy: INSTICC. <http://www.icissp.org/?y=2016>. Accessed: 2016-04-20., 2016.

ANDRADE, E. R.; SIMPLICIO JR, M. A.; BARRETO, P. S. L. M.; SANTOS, P. C. F. d. Lyra2: efficient password hashing with high security against time-memory trade-offs. IEEE Transactions on Computers, PP, n. 99, 2016. ISSN 0018-9340. See also <http://eprint.iacr.org/2015/136>.

ANDREEVA, E.; MENNINK, B.; PRENEEL, B. The Parazoa Family: Generalizing the Sponge Hash Functions. 2011. Cryptology ePrint Archive, Report 2011/028. <http://eprint.iacr.org/2011/028> . Accessed: 2014-07-18.

AOSP. Android Security Overview. 2012. Android Open Source Project $<$ http://source.android.com/tech/security/index.html>. Accessed: 2015-02-13.

Apple. iOS Security. U.S., 2012. < https://www.apple.com/br/ipad/business/docs/ iOS_Security_Oct12.pdf $>$. Accessed: 2015-06-08.

AUMASSON, J.-P.; FISCHER, S.; KHAZAEI, S.; MEIER, W.; RECHBERGER, C. New features of latin dances: Analysis of Salsa, ChaCha, and Rumba. In: Fast Software Encryption. Berlin, Heidelberg: Springer-Verlag, 2008. v. 5084, p. 470-488. ISBN 978-3-540-71038-7. 
AUMASSON, J.-P.; GUO, J.; KNELLWOLF, S.; MATUSIEWICZ, K.; MEIER, W. Differential and Invertibility Properties of BLAKE. In: HONG, S.; IWATA, T. (Ed.). Fast Software Encryption. Berlin, Germany: Springer Berlin Heidelberg, 2010, (Lecture Notes in Computer Science, v. 6147). p. 318-332. ISBN 978-3-642-13857-7. See also < http://eprint.iacr.org/2010/043>.

AUMASSON, J.-P.; HENZEN, L.; MEIER, W.; PHAN, R. SHA-3 proposal $B L A K E$ (version 1.3). 2010. <https://131002.net/blake/blake.pdf $>$.

AUMASSON, J.-P.; JOVANOVIC, P.; NEVES, S. NORX: Parallel and Scalable AEAD. In: KUTYLOWSKI, M.; VAIDYA, J. (Ed.). Computer Security ESORICS 2014. Berlin, Germany: Springer International Publishing, 2014, (LNCS, v. 8713). p. 19-36. ISBN 978-3-319-11211-4. See also <https://norx.io/>.

. Analysis of NORX: Investigating Differential and Rotational Properties.

In: ARANHA, D. F.; MENEZES, A. (Ed.). Progress in Cryptology LATINCRYPT 2014. Berlin, Germany: Springer International Publishing, 2015, (LNCS, v. 8895). p. 306-324. ISBN 978-3-319-16294-2. See also $<$ https://eprint.iacr.org/2014/317>.

AUMASSON, J.-P.; NEVES, S.; WILCOX-O'HEARN, Z.; WINNERLEIN, C. BLAKE2: simpler, smaller, fast as MD5. 2013. < https://blake2.net/>. Accessed: 2014-11-21.

BELLARE, M.; RISTENPART, T.; TESSARO, S. Multi-instance Security and Its Application to Password-Based Cryptography. In: SAFAVI-NAINI, R.; CANETTI, R. (Ed.). Advances in Cryptology - CRYPTO 2012. Berlin, Germany: Springer Berlin Heidelberg, 2012, (LNCS, v. 7417). p. 312-329. ISBN 978-3-642-32008-8.

BERNSTEIN, D. J. Cache-timing attacks on AES. Chicago, 2005. $<$ http://cr.yp.to/antiforgery/cachetiming-20050414.pdf $>$. Accessed: 2014-07-01.

. The Salsa20 family of stream ciphers. In: ROBSHAW, M.; BILLET, O. (Ed.). New Stream Cipher Designs. Berlin, Heidelberg: Springer-Verlag, 2008. p. 84-97. ISBN 978-3-540-68350-6.

BERTOnI, G.; DAEMEN, J.; PEETERS, M.; ASSCHE, G. V. Sponge functions. 2007. (ECRYPT Hash Function Workshop 2007). < http:

//sponge.noekeon.org/SpongeFunctions.pdf $>$. Accessed: 2015-06-09.

. Cryptographic sponge functions - version 0.1. 2011. < http://sponge. noekeon.org/CSF-0.1.pdf>. Accessed: 2015-06-09.

. The Keccak SHA-3 submission. 2011. Submission to NIST (Round 3). $<$ http://keccak.noekeon.org/Keccak-submission-3.pdf > . Accessed: 2015-06-09.

BIRYUKOV, A.; DINU, D.; KHOVRATOVICH, D. Argon2: the memoryhard function for password hashing and other applications. v1.3 of argon2. Luxembourg, 2016. < https://github.com/P-H-C/phc-winner-argon2/blob/master/ argon2-specs.pdf $>$. Accessed: 2016-04-20. 
BOnNeAU, J.; HeRley, C.; OORSCHOT, P. C. V.; STAJANO, F. The Quest to Replace Passwords: A Framework for Comparative Evaluation of Web Authentication Schemes. In: Security and Privacy (SP), 2012 IEEE Symposium on. San Francisco, CA: IEEEXplore, 2012. p. 553-567. ISSN 1081-6011.

BROZ, M. Another PHC candidates "mechanical" tests - Public archives of PHC list. 2014. < http://article.gmane.org/gmane.comp.security.phc/2237>. Accessed: 2014-11-27.

CAPCOM. Blanka - Capcom Database. 2015. < http://capcom.wikia.com/wiki/ Blanka >. Accessed: 2015-12-18.

CHAKRABARTI, S.; SINGBAL, M. Password-based authentication: Preventing dictionary attacks. Computer, v. 40, n. 6, p. 68-74, june 2007. ISSN 0018-9162.

CHANG, S.; PERLNER, R.; BURR, W. E.; TURAN, M. S.; KELSEY, J. M.; PAUL, S.; BASSHAM, L. E. Third-Round Report of the SHA-3 Cryptographic Hash Algorithm Competition. Washington, DC, USA: US Department of Commerce, National Institute of Standards and Technology, 2012. <http://nvlpubs.nist.gov/nistpubs/ir/2012/NIST.IR.7896.pdf>. Accessed: 2015-03-06.

CHUNG, E. S.; MILDER, P. A.; HOE, J. C.; MAI, K. Single-Chip Heterogeneous Computing: Does the Future Include Custom Logic, FPGAs, and GPGPUs? In: Proc. of the 43rd Annual IEEE/ACM International Symposium on Microarchitecture. Washington, DC, USA: IEEE Computer Society, 2010. (MICRO'43), p. 225-236. ISBN 978-0-7695-4299-7.

CONKLIN, A.; DIETRICH, G.; WALZ, D. Password-based authentication: A system perspective. In: Proc. of the 37th Annual Hawaii International Conference on System Sciences (HICSS'04). Washington, DC, USA: IEEE Computer Society, 2004. (HICSS'04, v. 7), p. 170-179. ISBN 0-7695-2056-1. $<$ http://dl.acm.org/citation.cfm?id $=962755.963150>$.

COOK, S. A. An Observation on Time-storage Trade off. In: Proc. of the 5th Annual ACM Symposium on Theory of Computing (STOC'r3). New York, NY, USA: ACM, 1973. p. 29-33.

COX, B. TwoCats (and SkinnyCat): A Compute Time and Sequential Memory Hard Password Hashing Scheme. v0. Chapel Hill, NC, 2014. $<$ https://password-hashing.net/submissions/specs/TwoCats-v0.pdf $>$. Accessed: 2014-12-11.

CREW, B. New carnivorous harp sponge discovered in deep sea. Nature, 2012. Available online: <http://www.nature.com/news/ new-carnivorous-harp-sponge-discovered-in-deep-sea-1.11789>. Accessed: 2013-12-21.

CRYPTO MINING. Updated Windows Binary of sgminer 5.1.1 With Fixed Lyra2Re Support - Crypto Mining Blog. 2015. <http://cryptomining-blog. 
com/4535-updated-windows-binary-of-sgminer-5-1-1-with-fixed-lyra2re-support/> . Accessed: 2015-05-06.

DADDA, L. Some schemes for parallel multipliers. Alta Frequenza, v. 34, p. 349-356, 1965.

. On parallel digital multipliers. Alta Frequenza, v. 45, p. 574-580, 1976.

DAEMEN, J.; RIJMEN, V. A new MAC construction ALRED and a specific instance ALPHA-mac. In: GILBERT, H.; HANDSCHUH, H. (Ed.). Fast Software Encryption - FSE'05. Berlin, Germany: Springer Berlin Heidelberg, 2005, (LNCS, v. 3557). p. 1-17. ISBN 978-3-540-26541-2.

. Refinements of the ALRED construction and MAC security claims. Information Security, IET, v. 4, n. 3, p. 149-157, 2010. ISSN 1751-8709.

DANDASS, Y. S. Using FPGAs to Parallelize Dictionary Attacks for Password Cracking. In: Proc. of the 41st Annual Hawaii International Conference on System Sciences (HICSS 2008). Waikoloa, HI: IEEEXplore, 2008. p. 485-485. ISSN 1530-1605.

DAY, T. Vertcoin (VTC) plans algorithm change to Lyra2 - Coinbrief. 2014. $<$ http://coinbrief.net/vertcoin-algorithm-change-lyra2/>. Accessed: 2015-05-06.

DÜRMUTH, M.; GÜNEYSU, T.; KASPER, M. Evaluation of Standardized Password-Based Key Derivation against Parallel Processing Platforms. In: Computer Security - ESORICS 2012. Berlin, Germany: Springer Berlin Heidelberg, 2012, (LNCS, v. 7459). p. 716-733. ISBN 978-3-642-33166-4.

DWORK, C.; NAOR, M.; WEE, H. Pebbling and Proofs of Work. In: Advances in Cryptology - CRYPTO 2005. Berlin, Germany: Springer Berlin Heidelberg, 2005. (LNCS, v. 3621), p. 37-54. ISBN 978-3-540-28114-6.

DZIEMBOWSKI, S.; KAZANA, T.; WICHS, D. Key-Evolution Schemes Resilient to Space-Bounded Leakage. In: Advances in Cryptology - CRYPTO 2011. Berlin, Germany: Springer Berlin Heidelberg, 2011. (LNCS, v. 6841), p. 335-353. ISBN 978-3-642-22791-2.

FLORENCIO, D.; HERLEY, C. A Large-scale Study of Web Password Habits. In: Proceedings of the 16th International Conference on World Wide Web. New York, NY, USA: ACM, 2007. p. 657-666. ISBN 978-1-59593-654-7.

FORLER, C.; LIST, E.; LUCKS, S.; WENZEL, J. Overview of the Candidates for the Password Hashing Competition - And Their Resistance Against Garbage-Collector Attacks. 2014. Cryptology ePrint Archive, Report 2014/881. $<$ http://eprint.iacr.org/2014/881>. Accessed: 2016-04-23.

FORLER, C.; LUCKS, S.; WENZEL, J. Catena: A Memory-Consuming Password Scrambler. 2013. Cryptology ePrint Archive, Report 2013/525. $<$ http://eprint.iacr.org/2013/525>. Accessed: 2014-03-03. 
. The Catena Password Scrambler: Submission to the Password Hashing Competition (PHC). v1. Weimar, Germany, 2014. < https://password-hashing.net/ submissions/specs/Catena-v1.pdf $>$. Accessed: 2014-10-09.

FOWERS, J.; BROWN, G.; COOKE, P.; STITT, G. A performance and energy comparison of FPGAs, GPUs, and multicores for sliding-window applications. In: Proc. of the ACM/SIGDA Internbational Symposium on Field Programmable Gate Arrays (FPGA'12). New York, NY, USA: ACM, 2012. p. 47-56. ISBN 978-1-4503-1155-7.

GAJ, K.; HOMSIRIKAMOL, E.; ROGAWSKI, M.; SHAHID, R.; SHARIF, M. U. Comprehensive Evaluation of High-Speed and Medium-Speed Implementations of Five SHA-3 Finalists Using Xilinx and Altera FPGAs. 2012. Cryptology ePrint Archive, Report 2012/368. < http://eprint.iacr.org/2012/368> . Accessed: 2014-03-07.

GONÇALVES, G. P. Github of Lyra2 implementation targeting modern CPU architectures and compilers. 2014. Github (C). < https://github.com/eggpi/lyra2 >. Accessed: 2016-04-21.

GONÇALVES, G. P.; ARANHA, D. F. Implementação eficiente em software da função lyra2 em arquiteturas modernas. In: Anais do XV Simpósio Brasileiro em Segurança da Informação e de Sistemas Computacionais. Florianópolis, SC, Brazil: Sociedade Brasileira de Computação (SBC), $<$ http://sbseg2015.univali.br/anais/AnaisSBSeg2015Completo.pdf $>$. Accessed: 2015-04-22, 2005. (SBSEG 2015), p. 388-397. ISSN 2176-0063.

GUO, J.; KARPMAN, P.; NIKOLIĆ, I.; WANG, L.; WU, S. Analysis of BLAKE2. In: Topics in Cryptology (CT-RSA 2014). Berlin, Germany: Springer International Publishing, 2014. (LNCS, v. 8366), p. 402-423. ISBN 978-3-319-04851-2. See also <https://eprint.iacr.org/2013/467>.

HALDERMAN, J.; SCHOEN, S.; HENINGER, N.; CLARKSON, W.; PAUL, W.; CALANDRINO, J.; FELDMAN, A.; APPELBAUM, J.; FELTEN, E. Lest we remember: cold-boot attacks on encryption keys. Commun. ACM, ACM, New York, NY, USA, v. 52, n. 5, p. 91-98, maio 2009. ISSN 0001-0782.

HATZIVASILIS, G.; PAPAEFSTATHIOU, I.; MANIFAVAS, C. Password Hashing Competition - Survey and Benchmark. 2015. Cryptology ePrint Archive, Report 2015/265. <http://eprint.iacr.org/2015/265>. Accessed: 2015-05-18.

HERLEY, C.; OORSCHOT, P. V.; PATRICK, A. Passwords: If We're So Smart, Why Are We Still Using Them? In: Financial Cryptography and Data Security. Berlin, Germany: Springer Berlin Heidelberg, 2009. (LNCS, v. 5628), p. 230-237. ISBN 978-3-642-03548-7.

ICISSP. Previous Awards. 2016. International Conference on Information Systems Security and Privacy - website. <http://www.icissp.org/PreviousAwards.aspx $>$. 2016-04-20. 
JI, L.; LIANGYU, X. Attacks on round-reduced BLAKE. 2009. Cryptology ePrint Archive, Report 2009/238. < http://eprint.iacr.org/2009/238> . Accessed: 2014-06-22.

KAKAROUNTAS, A. P.; MICHAIL, H.; MILIDONIS, A.; GOUTIS, C. E.; THEODORIDIS, G. High-Speed FPGA Implementation of Secure Hash Algorithm for IPSec and VPN Applications. The Journal of Supercomputing, v. 37, n. 2, p. 179-195, 2006. ISSN 0920-8542.

KALISKI, B. PKCS\#5: Password-Based Cryptography Specification version 2.0 (RFC 2898). RSA Laboratories. Cambridge, MA, USA, 2000. $<$ http://tools.ietf.org/html/rfc2898>. Accessed: 2014-03-12.

KELSEY, J.; SCHNEIER, B.; HALL, C.; WAGNER, D. Secure Applications of Low-Entropy Keys. In: Proc. of the 1st International Workshop on Information Security. London, UK, UK: Springer-Verlag, 1998. (ISW '97), p. 121-134. ISBN 3-540-64382-6.

KHOVRATOVICH, D.; BIRYUKOV, A.; GROBSCHÄDL, J. Tradeoff cryptanalysis of password hashing schemes. 2014. PasswordsCon'14. See also $<$ https://www.cryptolux.org/images/4/4f/PHC-overview.pdf $>$.

KHRONOS GROUP. The OpenCL Specification - Version 1.2. Beaverton, OR, USA, 2012. <www.khronos.org/registry/cl/specs/opencl-1.2.pdf>. Accessed: 2014-11-11.

LENGAUER, T.; TARJAN, R. E. Asymptotically Tight Bounds on Time-space Trade-offs in a Pebble Game. J. ACM, ACM, New York, NY, USA, v. 29, n. 4, p. 1087-1130, oct 1982. ISSN 0004-5411.

MARECHAL, M. Advances in password cracking. Journal in Computer Virology, Springer-Verlag, v. 4, n. 1, p. 73-81, 2008. ISSN 1772-9890.

MENEZES, A. J.; OORSCHOT, P. C. V.; VANSTONE, S. A.; RIVEST, R. L. Handbook of Applied Cryptography. 1. ed. Boca Raton, FL, USA: CRC Press, 1996. ISSN 0-8493-8523-7.

MING, M.; QIANG, H.; ZENG, S. Security Analysis of BLAKE-32 Based on Differential Properties. In: IEEE. 2010 International Conference on Computational and Information Sciences (ICCIS). Chengdu, 2010. p. 783-786.

MOWERY, K.; KEELVEEDHI, S.; SHACHAM, H. Are AES x86 Cache Timing Attacks Still Feasible? In: Proc.s of the 2012 ACM Workshop on Cloud Computing Security Workshop (CCSW'12). New York, NY, USA: ACM, 2012. p. 19-24. ISBN 978-1-4503-1665-1.

NEVES, S. Re: A review per day - Lyra2 - Public archives of PHC list. 2014. $<$ http://article.gmane.org/gmane.comp.security.phc/2045>. Accessed: 2014-12-20.

. Re: Compute time hardness (pwxform,blake,blamka). 2015. < http:

//article.gmane.org/gmane.comp.security.phc/2696>. Accessed: 2016-04-22. 
NIST. Federal Information Processing Standard (FIPS 197) - Advanced Encryption Standard (AES). National Institute of Standards and Technology, U.S. Department of Commerce. Gaithersburg, MD, USA, 2001. < http: //csrc.nist.gov/publications/fips/fips197/fips-197.pdf > . Accessed: 2016-04-15.

. Special Publication SP 800-38A - Recommendations for Block Cipher Modes of Operation, Methods and Techniques. National Institute of Standards and Technology, U.S. Department of Commerce. Gaithersburg, MD, USA, 2001. $<$ http://csrc.nist.gov/publications/nistpubs/800-38a/sp800-38a.pdf $>$. Accessed: 2016-04-15.

- Federal Information Processing Standard (FIPS PUB 180-2)

- Secure Hash Standard (SHS). National Institute of Standards and Technology, U.S. Department of Commerce. Gaithersburg, MD, USA, 2002. <http://csrc.nist.gov/publications/fips/fips180-2/fips180-2.pdf > Accessed: 2015-06-25.

. Federal Information Processing Standard (FIPS PUB 198) - The Keyed-Hash Message Authentication Code. National Institute of Standards and Technology, U.S. Department of Commerce. Gaithersburg, MD, USA, 2002. <http://csrc.nist.gov/publications/fips/fips198/fips-198a.pdf $>$. Accessed: 2014-04-28.

. Special Publication 800-18 - Recommendation for Key Derivation Using Pseudorandom Functions. National Institute of Standards and Technology, U.S. Department of Commerce. Gaithersburg, MD, USA, 2009. $<$ http://csrc.nist.gov/publications/nistpubs/800-108/sp800-108.pdf $>$. Accessed: 2013-11-02.

Special Publication 800-63-1 - Electronic Authentication Guideline.

National Institute of Standards and Technology, U.S. Department of Commerce. Gaithersburg, MD, USA, 2011. < http://csrc.nist.gov/publications/nistpubs/ 800-63-1/SP-800-63-1.pdf $>$. Accessed: 2014-03-30.

NVIDIA. Tesla Kepler Family Product Overview. 2012. < http://www.nvidia.com/ content/tesla/pdf/Tesla-KSeries-Overview-LR.pdf > . Accessed: 2013-10-03.

. CUDA C Programming Guide (v6.5). 2014. < http://docs.nvidia.com/ cuda/cuda-c-programming-guide/>. Accessed: 2013-10-03.

PERCIVAL, C. Cache missing for fun and profit. In: Proc. of BSDCan 2005. Ottawa, Canada: University of Ottawa, 2005.

. Stronger key derivation via sequential memory-hard functions. In: BSDCan 2009 - The Technical BSD Conference. Ottawa, Canada: University of Ottawa, 2009. See also: <http://www.bsdcan.org/2009/schedule/attachments/87_ scrypt.pdf $>$. Accessed: 2013-12-09.

PESLYAK, A. yescrypt - a Password Hashing Competition submission. v1. Moscow, Russia, 2015. < https://password-hashing.net/submissions/specs/ yescrypt-v0.pdf $>$. Accessed: 2015-05-22. 
PHC. Password Hashing Competition. 2013. < https://password-hashing.net/>. Accessed: 2015-05-06.

. Github of Argon2. 2015. Github (C). < https://github.com/p-h-c/ phc-winner-argon2>. Accessed: 2016-04-22.

. Password Hashing Competition. 2015. < https://password-hashing.net/ \#phc $>$. Accessed: 2016-04-23.

. PHC status report. 2015. < https://password-hashing.net/report-finalists. html $>$. Accessed: 2016-04-23.

. Public archives of PHC list. 2015. < http://dir.gmane.org/gmane.comp. security.phc $>$. Accessed: 2015-05-18.

PHC wiki. Password Hashing Competition - wiki. 2014. < https: //password-hashing.net/wiki/>. Accessed: 2015-05-06.

PROVOS, N.; MAZIĖRES, D. A future-adaptable password scheme. In: Proc. of the FREENIX track: 1999 USENIX annual technical conference. Monterey, California, USA: USENIX, 1999.

RISTENPART, T.; TROMER, E.; SHACHAM, H.; SAVAGE, S. Hey, You, Get off of My Cloud: Exploring Information Leakage in Third-party Compute Clouds. In: Proc.s of the 16th ACM Conference on Computer and Communications Security. New York, NY, USA: ACM, 2009. (CCS '09), p. 199-212. ISBN 978-1-60558-894-0.

RIVEST, R. L.; SHAMIR, A.; ADLEMAN, L. A method for obtaining digital signatures and public-key cryptosystems. Commun. ACM, ACM, New York, NY, USA, v. 21, n. 2, p. 120-126, Feb 1978. ISSN 0001-0782.

SCHNEIER, B. Description of a new variable-length key, 64-bit block cipher (Blowfish). In: Fast Software Encryption, Cambridge Security Workshop. London, UK: Springer-Verlag, 1994. p. 191-204. ISBN 3-540-58108-1.

SCIENGINES. RIVYERA S3-5000. 2013. < http://www.sciengines.com/products/ discontinued/rivyera-s3-5000.html>. Accessed: 2015-05-02.

RIVYERA V7-2000T. 2013. <http://sciengines.com/products/

computers-and-clusters/v72000t.html $>$. Accessed: 2015-05-02.

SHAND, M.; BERTIN, P.; VUILlEMIN, J. Hardware Speedups in Long Integer Multiplication. In: Proceedings of the Second Annual ACM Symposium on Parallel Algorithms and Architectures. New York, NY, USA: ACM, 1990. (SPAA'90), p. 138-145. ISBN 0-89791-370-1.

SIMPLICIO JR, M. A. Message Authentication Algorithms for Wireless Sensor Networks. Tese (Doutorado) — Escola Politécnica da Universidade de São Paulo (Poli-USP), São Paulo, September 2010. Available from: $<$ http://www.teses.usp.br/teses/disponiveis/3/3141/tde-11082010-114456/>. Accessed: 2015-03-17. 
SIMPLICIO JR, M. A. Re: Competition process. 2015. < http://article.gmane. org/gmane.comp.security.phc/2784>. Accessed: 2016-04-23.

SIMPliCIO JR, M. A.; BARBUDA, P.; BARRETO, P. S. L. M.; CARVALHO, T.; MARGI, C. The Marvin Message Authentication Code and the LetTerSoup Authenticated Encryption Scheme. Security and Communication Networks, v. 2, p. 165-180, 2009.

SIMPLICIO JR, M. A.; BARRETO, P. S. L. M. Revisiting the Security of the AlRed Design and Two of Its Variants: Marvin and LetterSoup. IEEE Transactions on Information Theory, v. 58, n. 9, p. 6223-6238, 2012.

SODERQUIST, P.; LEESER, M. An area/performance comparison of subtractive and multiplicative divide/square root implementations. In: Proc. of the 12th Symposium on Computer Arithmetic, 1995. Bath: IEEEXplore, 1995. p. $132-139$.

SOLAR DESIGNER. New developments in password hashing: ROM-port-hard functions. 2012. <http://www.openwall. com/presentations/ZeroNights2012-New-In-Password-Hashing/

ZeroNights2012-New-In-Password-Hashing.pdf> . Accessed: 2013-03-23.

SPRENGERS, M. GPU-based Password Cracking: On the Security of Password Hashing Schemes regarding Advances in Graphics Processing Units. Dissertação (Mestrado) — Radboud University Nijmegen, 2011. $<$ http://www.ru.nl/publish/pages/578936/thesis.pdf > . Accessed: 2014-02-12.

SU, B.; WU, W.; WU, S.; DONG, L. Near-Collisions on the Reduced-Round Compression Functions of Skein and BLAKE. In: Cryptology and Network Security. Berlin, Germany: Springer Berlin Heidelberg, 2010, (LNCS, v. 6467). p. 124-139. ISBN 978-3-642-17618-0.

SYNOPSYS. Design Compiler 2010. 2010. < http://www.synopsys.com/Tools/ Implementation/RTLSynthesis/DesignCompiler/Pages/default.aspx $>$. Accessed: 2016-04-25.

TERADA, R. Segurança de dados: Criptografia em redes de computador. 2, revisada e ampliada. ed. São Paulo, SP, Brazil: Blucher, 2008. ISBN 978-85-212-0439-8.

THOMAS, S. battcrypt (Blowfish All The Things). v0. Lisle, IL, USA, 2014. $<$ https://password-hashing.net/submissions/specs/battcrypt-v0.pdf $>$. Accessed: 2015-05-18.

TRENDFORCE. DRAM Contract Price (Aug.1 2016). 2016.

Http://www.trendforce.com/price (visited on Aug.1, 2016).

TRUECRYPT. TrueCrypt: Free open-source on-the-fly encryption Documentation. 2012. <http://www.truecrypt.org/docs/>. Accessed: 2014-09-01. 
WALLIS, W. D.; GEORGE, J. Introduction to Combinatorics. Florence, Kentucky, USA: Taylor \& Francis, 2011. (Discrete Mathematics and Its Applications). ISBN 9781439806234.

WAZLAWICK, R. S. Metodologia de pesquisa para ciência da computação. Rio de Janeiro: Elsevier, 2008. 184 p. ISSN 978-85-352-3522-7.

WEIR, M.; AGGARWAL, S.; MEDEIROS, B. d.; GLODEK, B. Password Cracking Using Probabilistic Context-Free Grammars. In: Proc. of the 30th IEEE Symposium on Security and Privacy. Washington, DC, USA: IEEE Computer Society, 2009. (SP'09), p. 391-405. ISBN 978-0-7695-3633-0.

WU, H. POMELO - A Password Hashing Algorithm (Version 3). v3. Nanyang Ave, Singapore, 2015. < https://password-hashing.net/submissions/specs/ POMELO-v3.pdf $>$. Accessed: 2015-05-18.

XILINX. ISE Design Suite. 2013. < http://www.xilinx.com/products/design-tools/ ise-design-suite.html $>$. Accessed: 2016-04-25.

. Vivado Design Suite. 2016. < http://www.xilinx.com/products/design-tools/ vivado.html $>$. Accessed: 2016-04-25.

YAO, F. F.; YIN, Y. L. Design and Analysis of Password-Based Key Derivation Functions. IEEE Transactions on Information Theory, v. 51, n. 9, p. 3292-3297, 2005. ISSN 0018-9448.

YUILL, J.; DENNING, D.; FEER, F. Using deception to hide things from hackers: Processes, principles, and techniques. Journal of Information Warfare, v. 5, n. 3, p. 26-40, 2006. ISSN 1445-3312. 


\section{APPENDIX A. NAMING CONVENTIONS}

The name "Lyra" comes from Chondrocladia lyra, a recently discovered type of sponge (CREW, 2012). While most sponges are harmless, this harp-like sponge is carnivorous, using its branches to ensnare its prey, which is then enveloped in a membrane and completely digested. The "two" suffix is a reference to its predecessor, Lyra (ALMEIDA et al., 2014), which displays many of Lyra2's properties hereby presented but has a lower resistance to attacks involving time-memory trade-offs. Lyra2's memory matrix displays some similarity with this species' external aspect, and we expect it to be at least as much aggressive against adversaries trying to attack it. (-)

Regarding the multiplication-hard sponge, its name came from an attempt to combine the name "Blake", which is the basis for the algorithm, with the letter "M", for indicating multiplications. A natural (?) answer for this combination was BlaMka, a misspelling of Blanka, the only avatar from the Street Fighter original game series (CAPCOM, 2015) that comes from Brazil and, as such, is a compatriot of this document's authors. :- 


\section{APPENDIX B. FURTHER CONTROLLING LYRA2'S BANDWIDTH USAGE}

Even though not part of Lyra2's core design, the algorithm could be adapted for allowing the user to control the number of rows involved in each iteration of the Visitation Loop. The reason is that, while Algorithm 5 suggests that a single row index besides row ${ }^{0}$ should be employed during the Setup and Wandering phases, this number could actually be controlled by a $\delta \geqslant 0$ parameter. Algorithm 5 can, thus, be seen as the particular case in which $\delta=1$, while the original Lyra is more similar (although not identical) to Lyra2 with $\delta=0$. This allows a better control over the algorithm's total memory bandwidth usage, so it can better match the bandwidth available at the legitimate platform.

This parameterization brings positive security consequences. For example, the number of rows written during the Wandering phase defines the speed in which the memory matrix is modified and, thus, the number of levels in the dependence tree discussed in Section 5.1.3.2. As a result, the $2 T$ observed in Equations 5.5 and 5.8 would actually become $(\delta+1) T$. The number of rows read, in turn, determines the tree's branching factor and, consequently, the probability that a previously discarded row will incur recomputations in Equation 5.3. With $\delta>1$, it is also possible to raise the Setup phase minimum memory usage above the $R / 2$ defined

by Lemma 1 . This can be accomplished by choosing visitation patterns for $r o w^{d \geqslant 2}$ that force the attacker to keep rows that, otherwise, could be discarded right after the middle of the Setup phase. One possible approach is, for example, to divide the revisitation window in the Setup phase into $\delta$ contiguous sub-windows, so each 
row ${ }^{d}$ revisits its own sub-window $\delta$ times. We note that this principle does not even need to be restricted to reads/writes on a same memory matrix: for example, one could add a row ${ }^{2}$ variable that indexes a Read-Only Memory chip attached to the device's platform and then only perform several reads (no writes) on this external memory, giving support to the "rom-port-hardness" concept discussed in (SOLAR DESIGNER, 2012).

Even though the security implications of having $\delta \geqslant 2$ may be of interest, the main disadvantage of this approach is that the higher number of rows picked potentially leads to performance penalties due to memory-related operations. This may oblige legitimate users to reduce the value of $T$ to keep Lyra2's running time below a certain threshold, which in turn would be beneficial to attack platforms having high memory bandwidth and able to mask memory latency (e.g., using idle cores that are waiting for input to run different password guesses). Indeed, according to our tests, we observed slow downs from more than $100 \%$ to approximately $50 \%$ with each increment of $\delta$ in the platforms used as testbed for our benchmarks (see Section 6). Therefore, the interest of supporting a customizable $\delta$ depends on actual tests made on the target platform, although we conjecture that this would only be beneficial with DRAM chips faster than those commercially available today. For this reason, in this document, we do not further explore the ability of adjusting $\delta$ to a value different from 1 . 


\section{APPENDIX C. AN ALTERNATIVE DESIGN FOR BLAMKA: AVOIDING LATENCY}

One drawback of the BlaMka permutation function as described in Section 4.4.1 is that it has no instruction parallelism. In platforms in which a legitimate user can execute instructions in parallel (e.g., in modern x86 processors), this can be a disadvantage, as the design will not take full advantage of the hardware available for the legitimate user. This potential disadvantage can be addressed with a quite simple tweak, which consists in replacing the addition $(+)$ introduced by BlaMka by a XOR operation.

This alternative structure is shown in Figure 18, which shows Blake2b's original $G$ function (on the left) and the two multiplication-hardened modified functions proposed in this work, $G_{\mathrm{tls}}$ as described in Section 4.4.1 and an alternative, XOR-based permutation hereby denoted called $G_{\mathrm{t} 1 \mathrm{~s}} \oplus$ aiming at reduced latency.

$$
\begin{aligned}
a & \leftarrow a+b \\
d & \leftarrow(d \oplus a) \ggg 32 \\
c & \leftarrow c+d \\
b & \leftarrow(b \oplus c) \ggg 24 \\
a & \leftarrow a+b \\
d & \leftarrow(d \oplus a) \ggg 16 \\
c & \leftarrow c+d \\
b & \leftarrow(b \oplus c) \ggg 63
\end{aligned}
$$

(a) Blake2 $G$ function.

$$
\begin{aligned}
a & \leftarrow a+b+2 \cdot \operatorname{lsw}(a) \cdot \operatorname{lsw}(b) \\
d & \leftarrow(d \oplus a) \ggg 32 \\
c & \leftarrow c+d+2 \cdot \operatorname{lsw}(c) \cdot \operatorname{lsw}(d) \\
b & \leftarrow(b \oplus c) \ggg 24 \\
a & \leftarrow a+b+2 \cdot \operatorname{lsw}(a) \cdot \operatorname{lsw}(b) \\
d & \leftarrow(d \oplus a) \ggg 16 \\
c & \leftarrow c+d+2 \cdot \operatorname{lsw}(c) \cdot \operatorname{lsw}(d) \\
b & \leftarrow(b \oplus c) \ggg 63
\end{aligned}
$$

(b) BlaMka's $G_{\mathrm{t} 1 \mathrm{~s}}$ function.

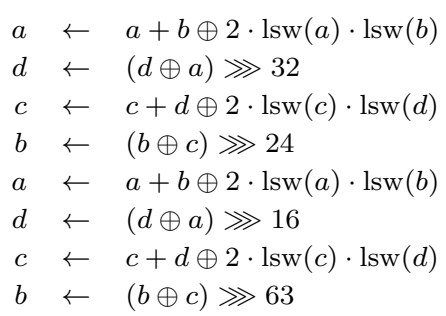

(c) BlaMka's $G_{\mathrm{t} 1 \mathrm{~s} \oplus}$ function.

Figure 18: Different permutations: Blake2b's original permutation (left), BlaMka's $G_{\mathrm{tls}}$ multiplication-hardened permutation (middle) and BlaMka's latency-oriented multiplication-hardened permutation $G_{\mathrm{tl} \mathbf{s}} \oplus$ (right). 
This trick was originally proposed on NORX - a Parallel and Scalable Authenticated Encryption Algorithm - (AUMASSON; JOVANOVIC; NEVES, 2014, Section 5.1), but during the PHC, Samuel Neves, one of the authors of this algorithm, suggested the possibility of also using this approach on BlaMka (NEVES, 2015).

With this modification, in principle, a developer can trade a few extra instructions by reduced latency, shortening the execution's pipeline, by implementing it as follows:

$$
\begin{aligned}
& t_{0} \leftarrow a+b \\
& t_{1} \leftarrow \operatorname{lsw}(a) \cdot \operatorname{lsw}(b)
\end{aligned}
$$

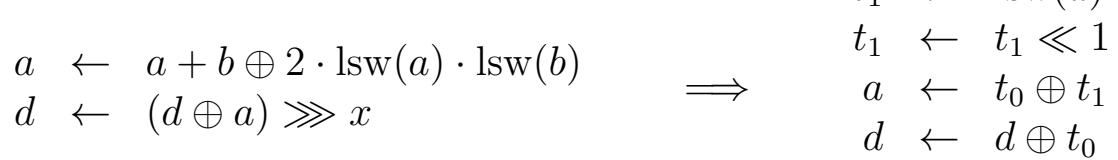

$$
\begin{aligned}
& d \leftarrow d \oplus t_{1} \\
& d \leftarrow d \ggg x
\end{aligned}
$$

This tweak saves up to 1 cycle per instruction sequence, leading to 4 instead of 5 cycles for $G_{\mathrm{t} 1 \mathrm{~s} \oplus}$, at the cost of 1 extra instruction (see Figure 19). In a sufficiently parallel architecture, this can save at least $4 \times 8$ cycles per round, since a single round of BlaMka has 8 calls to its underlying permutation.

In our initial measurements, this modification represented a somewhat modest gain of performance in legitimate platforms, which reached up to 6\% in Lyra2 when compared with BlaMka using the hereby adopted $G_{\mathrm{t} 1 \mathrm{~s}}$ permutation. However, our tests revealed that the ability to obtain this gain would heavily depend on the target architecture, coding, compiler, and type of vectorization. Therefore, as it would be difficult to have the same gain in practice for all legitimate platforms, while attackers could very well use dedicated hardware to take more advantage of it than legitimate users, we preferred not to adopt this approach in the design of the BlaMka sponge. 


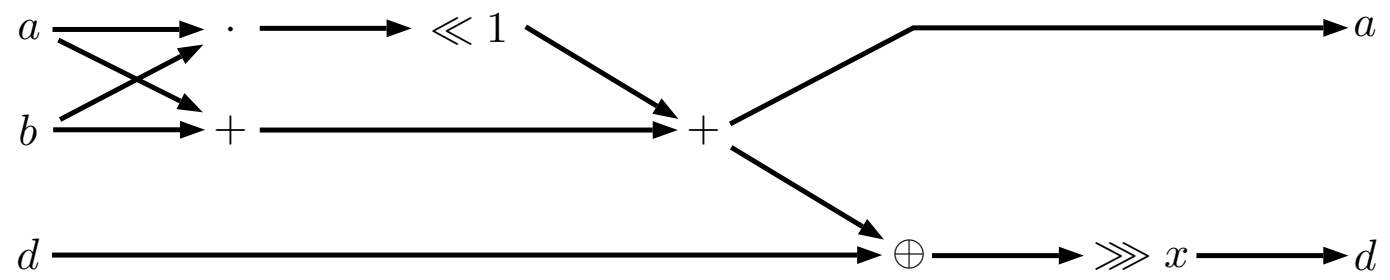

(a) Naïve implementation of BlaMka's $G_{\mathrm{t} 1 \mathrm{~s}}$ instruction sequence.

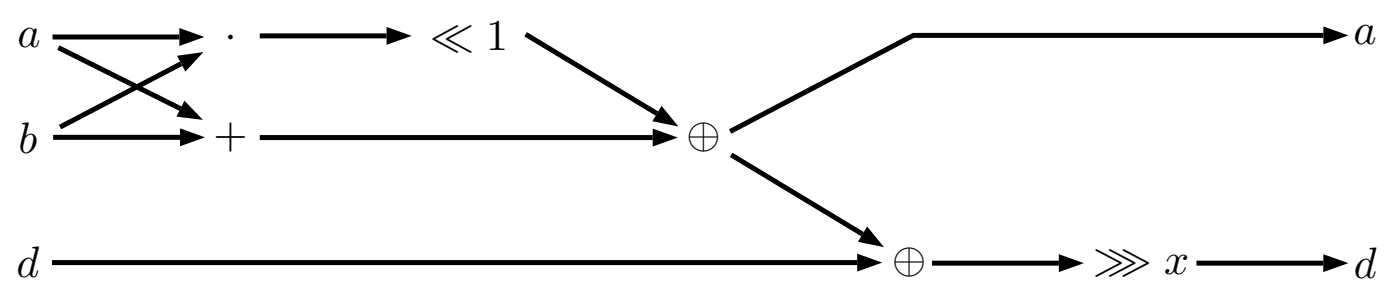

(b) Naïve implementation of BlaMka $G_{\mathrm{t} 1 \mathrm{~s} \oplus}$ instruction sequence.

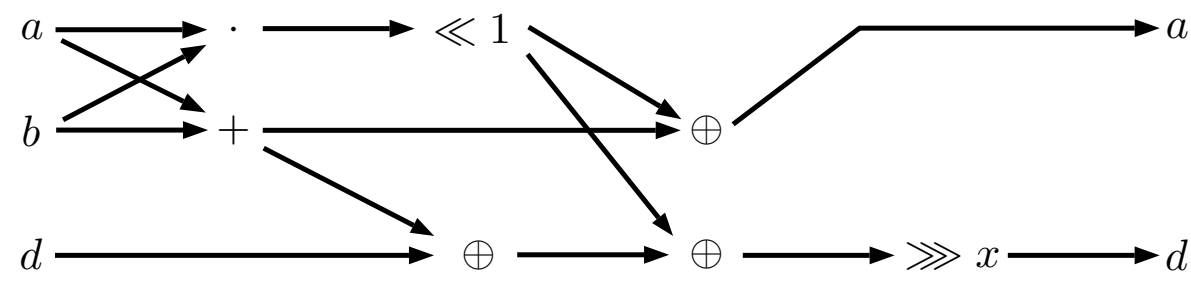

(c) Latency-oriented version of BlaMka's $G_{\mathrm{t} 1 \mathrm{~s} \oplus}$ instruction sequence.

Figure 19: Improving the latency of $G$. 